\title{
STARK POINTS AND $p$-ADIC ITERATED INTEGRALS ATTACHED TO MODULAR FORMS OF WEIGHT ONE
}

\author{
HENRI DARMON ${ }^{1}$, ALAN LAUDER $^{2}$ and VICTOR ROTGER ${ }^{3}$ \\ ${ }^{1}$ McGill University, Montreal, Canada; \\ email: darmon@math.mcgill.ca \\ ${ }^{2}$ University of Oxford, UK; \\ email: lauder@maths.ox.ac.uk \\ ${ }^{3}$ Universitat Politècnica de Catalunya, Barcelona, Spain; \\ email: victor.rotger@upc.edu
}

Received 16 December 2014; accepted 4 September 2015

To our families

\begin{abstract}
Let $E$ be an elliptic curve over $\mathbb{Q}$, and let $\varrho_{b}$ and $\varrho_{\sharp}$ be odd two-dimensional Artin representations for which $\varrho_{b} \otimes \varrho_{\sharp}$ is self-dual. The progress on modularity achieved in recent decades ensures the existence of normalized eigenforms $f, g$, and $h$ of respective weights two, one, and one, giving rise to $E, \varrho_{b}$, and $\varrho_{\sharp}$ via the constructions of Eichler and Shimura, and of Deligne and Serre. This article examines certain $p$-adic iterated integrals attached to the triple $(f, g, h)$, which are $p$-adic avatars of the leading term of the Hasse-Weil-Artin $L$-series $L\left(E, \varrho_{b} \otimes \varrho_{\sharp}, s\right)$ when it has a double zero at the centre. A formula is proposed for these iterated integrals, involving the formal group logarithms of global points on E-referred to as Stark points - which are defined over the number field cut out by $\varrho_{b} \otimes \varrho_{\sharp}$. This formula can be viewed as an elliptic curve analogue of Stark's conjecture on units attached to weight-one forms. It is proved when $g$ and $h$ are binary theta series attached to a common imaginary quadratic field in which $p$ splits, by relating the arithmetic quantities that arise in it to elliptic units and Heegner points. Fast algorithms for computing $p$-adic iterated integrals based on Katz expansions of overconvergent modular forms are then exploited to gather numerical evidence in more exotic scenarios, encompassing Mordell-Weil groups over cyclotomic fields, ring class fields of real quadratic fields (a setting which may shed light on the theory of Stark-Heegner points attached to Shintani-type cycles on $\mathcal{H}_{p} \times \mathcal{H}$ ), and extensions of $\mathbb{Q}$ with Galois group a central extension of the dihedral group $D_{2 n}$ or of one of the exceptional subgroups $A_{4}, S_{4}$, and $A_{5}$ of $\mathbf{P G L}_{2}(\mathbb{C})$.
\end{abstract}

2010 Mathematics Subject Classification: 11G05 (primary); 11F11, 11G40 (secondary)

(c) The Author(s) 2015. This is an Open Access article, distributed under the terms of the Creative Commons Attribution licence (http://creativecommons.org/licenses/by/4.0/), which permits unrestricted re-use, distribution, and reproduction in any medium, provided the original work is properly cited. 


\section{CONTENTS}

Introduction 2

1. Overconvergent weight-one forms and Gross-Stark units 16

2. Iterated integrals and the Garrett-Hida $p$-adic $L$-function 25

3. Heegner points and theta series of imaginary quadratic fields 34

4. Stark-Heegner points and theta series of real quadratic fields 60

5. Stark points over $A_{4}, S_{4}$ or $A_{5}$ extensions and exotic weight-one forms $\quad 68$

6. Stark points attached to odd two-dimensional Artin representations 84

7. Stark points over cyclotomic fields and Eisenstein series 86 Appendix. Digest of numerical examples $\quad 92$ References $\quad 93$

\section{Introduction}

The purpose of this article is to formulate an analogue of the Stark conjecture on units in number fields in the setting of global points on elliptic curves. We prove this 'elliptic Stark conjecture' in some cases and provide numerical evidence in its support in many others. It is offered as a more constructive alternative to the Birch and Swinnerton-Dyer (BSD) conjecture, since it often allows the efficient analytic computation of $p$-adic logarithms of global points. In contrast, the BSD conjecture (and its $p$-adic variant formulated by Mazur, Tate, and Teitelbaum) gives the ( $p$-adic) heights of such points, from which it appears computationally more difficult to recover the points themselves [Si]. Our conjecture gives a unifying perspective on the two currently known constructions of global points on elliptic curves over $\mathbb{Q}$ (Heegner points and the still conjectural Stark-Heegner points attached to real quadratic cycles on $\mathcal{H}_{p} \times \mathcal{H}$ ), and suggests a possible global underpinning for the latter. It also yields conjectural constructions and explicit formulae, in situations of rank one and two, for global points over cyclotomic fields, abelian extensions of quadratic fields which are not necessarily anticyclotomic, and extensions of $\mathbb{Q}$ with Galois group a central extension of $A_{4}, S_{4}$, or $A_{5}$.

Let $E$ be an elliptic curve over $\mathbb{Q}$ with Galois representation $V_{p}(E):=$ $H_{\mathrm{et}}^{1}\left(E_{\overline{\mathbb{Q}}}, \mathbb{Q}_{p}(1)\right)$, and let

$$
\varrho: G_{\mathbb{Q}} \longrightarrow \operatorname{Aut}\left(V_{\varrho}\right) \simeq \mathbf{G L}_{n}(L), \quad \operatorname{dim}_{L}\left(V_{\varrho}\right)=n \geqslant 1
$$

be an Artin representation-that is, a continuous Galois representation factoring through a finite quotient $\mathrm{Gal}(H / \mathbb{Q})$ of $G_{\mathbb{Q}}$-with coefficients in some finite extension $L \subset \mathbb{C}$ of $\mathbb{Q}$. The Hasse-Weil-Artin L-series $L(E, \varrho, s)$ of $E$ 
twisted by $\varrho$ is the $L$-function of the compatible system $V_{p}(E) \otimes V_{\varrho}$ of $p$-adic representations of $G_{\mathbb{Q}}$. It is defined on the right half-plane $\operatorname{Re}(s)>3 / 2$ by an absolutely convergent Euler product of degree $2 n$, and is expected to admit an analytic continuation to the whole complex plane. Under this assumption, the analytic rank of the pair $(E, \varrho)$ is defined to be

$$
r_{\text {an }}(E, \varrho):=\operatorname{ord}_{s=1} L(E, \varrho, s) .
$$

A natural Galois equivariant refinement of the Birch and Swinnerton-Dyer conjecture predicts that

$$
r_{\text {an }}(E, \varrho) \stackrel{?}{=} r(E, \varrho),
$$

where $r(E, \varrho)$ is the multiplicity of $V_{\varrho}$ as a subrepresentation of $E(H) \otimes L$ when $\varrho$ is irreducible, defined for general $\varrho$ by

$$
r(E, \varrho):=\operatorname{dim}_{L} \operatorname{Hom}_{G_{\mathbb{Q}}}\left(V_{\varrho}, E(H) \otimes L\right) .
$$

Equality (1) has been proved when $r_{\mathrm{an}}(E, \varrho)=0$ and (in roughly chronological order)

(I) $\varrho$ is the odd self-dual two-dimensional Galois representation induced from a ring class character of an imaginary quadratic field, by a series of works building on the breakthroughs of Gross and Zagier, and of Kolyvagin;

(II) $\varrho$ is a Dirichlet character, by the work of Kato [Kato];

(III) $\varrho$ is an odd irreducible two-dimensional Galois representation satisfying some mild restrictions, by [BDR2];

(IV) $\varrho$ is an irreducible constituent of the tensor product $\varrho_{b} \otimes \varrho_{\sharp}$ of two odd irreducible two-dimensional Galois representations which is self-dual and satisfies some other mild restrictions, by [DR2].

The setting where $r_{\mathrm{an}}(E, \varrho)>0$ raises the challenge of constructing nonzero elements in

$$
E(H)_{L}^{\varrho}:=\sum_{\varphi} \varphi\left(V_{\varrho}\right), \quad \text { where } \varphi \text { runs over a basis of } \operatorname{Hom}_{G_{\mathbb{Q}}}\left(V_{\varrho}, E(H) \otimes L\right) .
$$

Devising strategies for constructing such global points is arguably the most fundamental issue surrounding the Birch and Swinnerton-Dyer conjecture. Yet very little is known in this direction beyond the setting alluded to in item I above, where the methods of Gross, Zagier, and Kolyvagin can also be exploited to establish (1) when $r_{\text {an }}(E, \varrho)=1$, by using Heegner points (as recalled in Section 3 below) to construct the predicted nontrivial elements of $E(H)_{L}^{\varrho}$. 
As explained in the survey [BCDDPR], the techniques used to analyse settings II, III, and IV can be organized under the common rubric of 'Euler systems of Garrett-Rankin-Selberg type'. The present work considers the same settings, but in the less well-understood scenario of 'analytic rank $\leqslant 2$ '. More precisely, it will be assumed that $\varrho$ is an irreducible constituent of the tensor product $\varrho_{b} \otimes \varrho_{\sharp}$, where $\varrho_{b}$ and $\varrho_{\sharp}$ are odd, two-dimensional, but not necessarily irreducible, Artin representations satisfying the self-duality condition

$$
\chi:=\operatorname{det}\left(\varrho_{b}\right)^{-1}=\operatorname{det}\left(\varrho_{\sharp}\right),
$$

and that $r_{\mathrm{an}}(E, \varrho)=1$ or 2 . The primary goal of this paper is to propose a conjectural $p$-adic analytic formula relating global points in $E(H)_{L}^{\varrho}$ to " $p$-adic iterated integrals' attached to an appropriate triple of modular forms, and to prove it in some cases.

The following general notation and background are required to formulate the 'elliptic Stark conjecture' (Conjecture ES) below. For any positive integer $N$, any Dirichlet character $\eta:(\mathbb{Z} / N \mathbb{Z})^{\times} \rightarrow \overline{\mathbb{Q}}^{\times}$, and any field $L$, write $S_{k}(N, \eta)_{L} \subseteq$ $M_{k}(N, \eta)_{L}$ for the spaces of classical cusp forms and modular forms of weight $k$, level $N$, and character $\eta$, with Fourier coefficients in $F$. (When $\eta$ is suppressed from the notation, it is to be understood that $\eta$ is the trivial character.)

Thanks to Wiles' proof of the Shimura-Taniyama conjecture, the elliptic curve $E$ is known to be modular: there is a normalized weight-two newform $f \in S_{2}\left(N_{f}\right)_{\mathbb{Q}}$, where $N_{f}$ is the conductor of $E$, satisfying

$$
L(f, s)=L(E, s) .
$$

The work of Khare and Wintenberger [KW] on Serre's modularity conjecture likewise implies that any odd, two-dimensional Artin representation arises from a classical (cuspidal or Eisenstein) normalized newform of weight one. More precisely, denoting by $N_{g}$ and $N_{h}$ the Artin conductors of $\varrho_{b}$ and $\varrho_{\sharp}$, there are weight-one newforms $g \in M_{1}\left(N_{g}, \chi^{-1}\right)_{L}$ and $h \in M_{1}\left(N_{h}, \chi\right)_{L}$ satisfying

$$
L(g, s)=L\left(\varrho_{b}, s\right), \quad L(h, s)=L\left(\varrho_{\sharp}, s\right) .
$$

Henceforth, it will be convenient to use $\varrho_{g}$ and $\varrho_{h}$ to designate $\varrho_{b}$ and $\varrho_{\sharp}$, to write

$$
\varrho_{g h}:=\varrho_{g} \otimes \varrho_{h}: \operatorname{Gal}(H / \mathbb{Q}) \longrightarrow \mathbf{S L}_{4}(L),
$$

and to denote by $V_{g}, V_{h}$, and $V_{g h}$ the $L$-vector spaces underlying $\varrho_{g}, \varrho_{h}$, and $\varrho_{g h}$, respectively.

When either $g$ or $h$ is an Eisenstein series, the Rankin-Selberg method yields the analytic continuation and functional equation for the $L$-function

$$
L\left(E, \varrho_{g h}, s\right)=L(f \otimes g \otimes h, s)
$$


relating its values at $s$ and $2-s$. This has been extended to the general case where both $g$ and $h$ are cusp forms, by a result of Garrett. The self-duality condition on $\varrho_{g h}$ implies that the root number $\varepsilon\left(E, \varrho_{g h}\right)$ that appears in this functional equation is \pm 1 , and that it determines the parity of $r_{\mathrm{an}}\left(E, \varrho_{g h}\right)$. Furthermore, it can be written as a product of local root numbers

$$
\varepsilon\left(E, \varrho_{g h}\right)=\prod_{v \mid N \cdot \infty} \varepsilon_{v}\left(E, \varrho_{g h}\right) \quad\left(\text { where } N:=\operatorname{lcm}\left(N_{f}, N_{g}, N_{h}\right)\right),
$$

and the local sign $\varepsilon_{\infty}\left(E, \varrho_{g h}\right)$ is always equal to 1 . The following hypothesis on the local root numbers $\varepsilon_{v}\left(E, \varrho_{g h}\right)$ at the non-Archimedean places, which is discussed in further detail in Section 2, shall be imposed throughout this article.

Hypothesis A (Local sign hypothesis). For all finite places $v \mid N$, the local signs $\varepsilon_{v}\left(E, \varrho_{g h}\right)$ are equal to +1 . In particular, the same is true of the global root number, and hence $L\left(E, \varrho_{g h}, s\right)$ vanishes to even order at $s=1$.

This hypothesis on the local sign is often satisfied: for instance, whenever $\operatorname{gcd}\left(N_{f}, N_{g}, N_{h}\right)=1$. Under Hypothesis A, the method of Garrett, Rankin, and Selberg relates the central critical value $L(f \otimes g \otimes h, 1)$ to the values of the trilinear form

$$
I: S_{2}(N)_{\mathbb{C}} \times M_{1}\left(N, \chi^{-1}\right)_{\mathbb{C}} \times M_{1}(N, \chi)_{\mathbb{C}} \longrightarrow \mathbb{C}
$$

defined by

$$
I(\breve{f}, \breve{g}, \breve{h}):=\langle\breve{f}, \breve{g} \breve{h}\rangle,
$$

where $\langle$,$\rangle denotes the Petersson scalar product on S_{2}(N)_{\mathbb{C}} \times M_{2}(N)_{\mathbb{C}}$. More precisely, for any integer $N$, let $\mathbb{T}_{N}$ denote the Hecke algebra generated by the good Hecke operators $T_{n}$ with $n \nmid N$. If $M$ is any $\mathbb{T}_{N}$-module and $\phi \in M$ is a simultaneous eigenvector for the action of $\mathbb{T}_{N}$, satisfying $T_{\ell} \phi=a_{\ell}(\phi) \phi$ for all $\ell \nmid N$, let $I_{\phi} \subset \mathbb{T}_{N}$ be the ideal associated to this system of Hecke eigenvalues, and denote by

$$
M[\phi]=\operatorname{Ker}\left(I_{\phi}\right)=\left\{m \in M: T_{\ell} m=a_{\ell}(\phi) m \text { for all } \ell \nmid N\right\}
$$

the corresponding eigenspace. By the work [HaKu] of Harris and Kudla, the restriction

$$
I_{f g h}: S_{2}(N)[f] \times M_{1}\left(N, \chi^{-1}\right)[g] \times M_{1}(N, \chi)[h] \longrightarrow \mathbb{C}
$$

of $I$ to the product of isotypic components for $f, g$, and $h$ is identically zero if and only if the central critical value $L(f \otimes g \otimes h, 1)$ vanishes. Since this article is solely interested in the setting where $\varrho_{g h}$ admits irreducible constituents $\varrho$ for which $L(E, \varrho, 1)=0$, the following hypothesis is also assumed throughout. 
Hypothesis B (Global vanishing hypothesis). The $L$-function $L\left(E, \varrho_{g h}, s\right)$ vanishes at $s=1$, and hence the trilinear form $I_{f g h}$ in (3) is identically zero.

Under Hypotheses $\mathrm{A}$ and $\mathrm{B}$, the $L$-function $L\left(E, \varrho_{g h}, s\right)$ vanishes to order at least two at $s=1$. When $L^{\prime \prime}\left(E, \varrho_{g h}, 1\right) \neq 0$, the elliptic Stark conjecture of this article expresses the values of certain $p$-adic iterated integrals attached to the triple $(f, g, h)$ in terms of the formal group logarithms of global points in the $\varrho_{g h}$-isotypic subspace $E(H)_{L}^{\varrho_{g h}}$ of $E(H) \otimes L$.

The definition of these $p$-adic iterated integrals is based on the theory of $p$-adic and overconvergent modular forms. Let $p$ be a prime number, and fix an embedding of $\overline{\mathbb{Q}}$ in $\overline{\mathbb{Q}}_{p}$. Let $\mathbb{C}_{p}$ denote the completion of $\overline{\mathbb{Q}}_{p}$, and let $H_{p}$ and $L_{p}$ denote the $p$-adic closure of $H$ and $L$ in $\mathbb{C}_{p}$ with respect to the fixed embedding. Assume that $p$ is chosen in such a way that

$$
\operatorname{ord}_{p}\left(N_{f}\right) \leqslant 1 \text { and } \quad p \nmid N_{g} N_{h} \text {, and set } N:=\text { the prime-to- } p \text { part of } N \text {. }
$$

(This hypothesis is made primarily for ease of exposition, and it would eventually be interesting to relax it.)

The symbols $S_{k}^{(p)}(N, \chi) \subset M_{k}^{(p)}(N, \chi)$ refer to the spaces of $p$-adic cusp forms (respectively modular forms) of weight $k$, level $N$, and character $\chi$, with coefficients in $\mathbb{C}_{p}$, while $S_{k}^{\text {oc }}(N, \chi) \subset M_{k}^{\text {oc }}(N, \chi)$ stand for the corresponding subspaces of overconvergent modular forms. The relations between these various spaces are summarized in the following sequence of natural inclusions of $\mathbb{C}_{p}$-vector spaces:

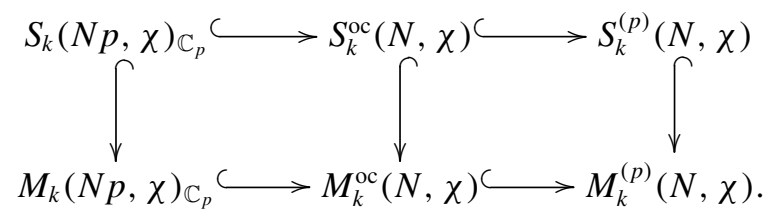

These spaces are all endowed with an action of the Hecke algebra $\mathbb{T}_{N}^{(p)}$ generated by the Hecke operators $T_{\ell}$ with $\ell \nmid N p$, and by the Hecke operator $U_{p}$. Let $e_{\text {ord }}:=\lim _{n} U_{p}^{n !}$ be Hida's ordinary idempotent, and write $M^{\text {ord }}=e_{\text {ord }} \cdot M$, for any $L_{p}\left[U_{p}\right]$-module $M$. While the spaces $S_{k}^{\text {oc }}(N, \chi)$ and $M_{k}^{\text {oc }}(N, \chi)$ are infinite dimensional over $\mathbb{C}_{p}$, their ordinary parts $S_{k}^{\text {oc,ord }}(N, \chi)$ and $M_{k}^{\text {oc,ord }}(N, \chi)$ are known to be finite dimensional. In fact, Hida's classicality theorem asserts that, for all $k \geqslant 2$, the natural inclusion

$$
M_{k}^{\text {ord }}(N p, \chi)_{\mathbb{C}_{p}} \hookrightarrow M_{k}^{\text {oc, ord }}(N, \chi)
$$

is an isomorphism. 
Serre's differential operator

$$
d=q \frac{d}{d q}: M_{k}^{(p)}(N, \chi) \longrightarrow M_{k+2}^{(p)}(N, \chi)
$$

on $p$-adic modular forms sends overconvergent forms of weight zero to overconvergent forms of weight two. For any $\breve{f} \in S_{2}^{\text {oc }}(N)$, the overconvergent modular function (or modular form of weight zero)

$$
\breve{F}:=d^{-1} \breve{f}:=\lim _{t \rightarrow-1} d^{t} \breve{f} \in S_{0}^{\mathrm{oc}}(N)
$$

is called the overconvergent primitive of $\breve{f}$. Here, the limit is taken over positive integers $t$ tending to -1 in weight space $\mathbb{Z} /(p-1) \mathbb{Z} \times \mathbb{Z}_{p}$. The Fourier expansions of $\breve{f}$ and $\breve{F}$ are related by

$$
\breve{f}(q)=\sum_{n \geqslant 1} a_{n}(\breve{f}) q^{n}, \quad \breve{F}(q)=\sum_{p \nmid n} \frac{a_{n}(\breve{f})}{n} q^{n} .
$$

For any $k \geqslant 2$, the $p$-adic iterated integral attached to the data

$$
(\breve{f}, \breve{\gamma}, \breve{h}) \in S_{2}(N p)_{L} \times M_{k}^{\text {ord }}(N p, \chi)_{L}^{\vee} \times M_{k}(N p, \chi)_{L}
$$

(in which the superscript $\vee$ denotes the $L$-linear dual) is defined to be

$$
\int_{\breve{\gamma}} \breve{f} \cdot \breve{h}:=\breve{\gamma}\left(e_{\text {ord }}(\breve{F} \cdot \breve{h})\right) \in \mathbb{C}_{p} .
$$

Expressions of this sort with $k \geqslant 2$ play an important role in [DR1, DR2], where they are related (when $\breve{f}, \breve{\gamma}$, and $\breve{h}$ are cuspidal eigenvectors) to the special values of Garrett-Hida triple-product $p$-adic $L$-functions and to the images of certain generalized Gross-Schoen diagonal cycles under the $p$-adic Abel-Jacobi map. When $\breve{\gamma}$ and/or $\breve{h}$ arise from weight-two Eisenstein series, these expressions are also related to $p$-adic regulators of Beilinson elements in the $K$-groups $K_{2}\left(X_{1}(N)\right)$ or in $K_{1}\left(X_{1}(N)^{2}\right)$ (see [BD2] and [BDR1], [BDR2], respectively).

On the computational side, using a method for computing with overconvergent modular forms via Katz expansions developed in [La1], the article [La2] describes an algorithm for the efficient numerical evaluation of these $p$-adic iterated integrals, and uses it to calculate certain Chow-Heegner points on the elliptic curve $E$ which were first defined and studied by Shouwu Zhang. These global points, which arise when $k \geqslant 2$ and $\breve{\gamma}$ and $\breve{h}$ are eigenvectors for $\mathbb{T}_{N}$ with the same eigenvalues, have a well-understood geometric provenance, and can also be calculated by complex analytic means following the strategy described in [DRS, DDLR]. 
The setting $k=1$ which is germane to the present work is significantly more mysterious: the $p$-adic iterated integrals (and, indeed, the modular forms $g$ and $h$ themselves) cease to enjoy geometric interpretations in terms of the cohomology of Kuga-Sato varieties. On a more technical level, the space $M_{1}^{\text {oc, ord }}(N, \chi)$, while still finite dimensional, may contain the space $M_{1}^{\text {ord }}(N p, \chi)_{\mathbb{C}_{p}}$ as a proper subspace. Fix a root $\alpha_{g}$ of the Hecke polynomial

$$
x^{2}-a_{p}(g) x+\chi^{-1}(p)=\left(x-\alpha_{g}\right)\left(x-\beta_{g}\right)
$$

attached to $g$ at $p$, and let $g_{\alpha} \in M_{1}\left(N p, \chi^{-1}\right)_{L}$ denote the corresponding $p$ stabilization. It is an eigenform for the Hecke algebra $\mathbb{T}_{N}^{(p)}$ satisfying

$$
U_{p} g_{\alpha}=\alpha_{g} \cdot g_{\alpha} .
$$

The weight-one form $g$ is said to be regular at $p$ if $\alpha_{g} \neq \beta_{g}$, that is, if $g$ admits two distinct $p$-stabilizations. Let $g_{\alpha}^{*}=g_{\alpha} \otimes \chi$ denote the newform of level $N_{g}$ corresponding to the twist of $g_{\alpha}$ by $\chi$, the inverse of its nebentype character.

If $\phi$ is a simultaneous eigenvector for either the Hecke algebra $\mathbb{T}_{N}^{(p)}$ or $\mathbb{T}_{N p}$, then, extending the definitions in (2), let

$$
M[\phi]:=\operatorname{Ker}\left(I_{\phi}\right), \quad M[[\phi]]=\bigcup_{n \geqslant 1} \operatorname{Ker}\left(I_{\phi}^{n}\right)
$$

denote the eigenspace and generalized eigenspace attached to its system of eigenvalues.

The following assumption is a crucial substitute for the classicality theorem when $k=1$.

Hypothesis C (Classicality property for $g_{\alpha}$ ). The cuspidal generalized eigenspace $S_{1}^{\text {oc,ord }}(N, \chi)_{\mathbb{C}_{p}}\left[\left[g_{\alpha}^{*}\right]\right]$ is nontrivial and consists entirely of classical forms.

When $g$ is a cusp form, Proposition 1.1 of Section 1.1 establishes, as an immediate consequence of the work of Cho and Vatsal [CV] and of Bellaïche and Dimitrov [BeDi], that Hypothesis $\mathrm{C}$ holds-that is, that the natural inclusion

$$
i_{g_{\alpha}}: S_{1}(N p, \chi)_{\mathbb{C}_{p}}\left[\left[g_{\alpha}^{*}\right]\right] \hookrightarrow S_{1}^{\text {oc,ord }}(N, \chi)\left[\left[g_{\alpha}^{*}\right]\right]
$$

is an isomorphism of $\mathbb{C}_{p}$-vector spaces-whenever $g$ satisfies the explicit condition (1) of Hypothesis $C^{\prime}$ below. (As discussed in Remark 1.2 of Section 1.1, this condition is expected to be necessary as well as sufficient.)

When $g$ is an Eisenstein series, Proposition 1.3 shows that the cuspidal generalized eigenspace $S_{1}^{\text {oc,ord }}(N, \chi)\left[\left[g_{\alpha}^{*}\right]\right]$ is nonzero, that is, $g_{\alpha}$ is a $p$-adic 
cusp form, if and only if $g$ satisfies condition (2) of Hypothesis $\mathrm{C}^{\prime}$ below. While the larger space $M_{1}^{\text {oc, ord }}(N, \chi)\left[\left[g_{\alpha}^{*}\right]\right]$ always contains nonclassical modular forms in this setting (whose Fourier expansions can be written down explicitly), it nonetheless seems plausible that $S_{1}^{\text {oc,ord }}(N, \chi)\left[\left[g_{\alpha}^{*}\right]\right]$ should consist solely of classical forms, that is, that the natural map

$$
i_{g_{\alpha}}: M_{1}(N p, \chi)_{\mathbb{C}_{p}}\left[\left[g_{\alpha}^{*}\right]\right] \cap S_{1}^{\text {oc, ord }}(N, \chi)\left[\left[g_{\alpha}^{*}\right]\right] \hookrightarrow S_{1}^{\text {oc,ord }}(N, \chi)\left[\left[g_{\alpha}^{*}\right]\right]
$$

should be an isomorphism. However, the analogue of the theorem of Bellaïche and Dimitrov for irregular weight-one Eisenstein points does not appear to be available in the literature, and therefore Hypothesis $\mathrm{C}$ could conceivably be stronger than condition (2) of Hypothesis $\mathrm{C}^{\prime}$ in the Eisenstein setting.

Motivated by this discussion, the following Hypothesis $\mathrm{C}^{\prime}$, which has the virtue of being entirely explicit, shall be assumed throughout along with Hypothesis C. (It is worth keeping in mind that both hypotheses are quite likely to be equivalent.)

HYPOTHESIS C' (Classicality property for $g_{\alpha}$, bis). The modular form $g$ satisfies one of the following explicit conditions.

(1) It is a cusp form which is regular at the prime $p$ (that is, $\varrho_{g}$ is irreducible and the Frobenius element at $p$ acts on it with two distinct eigenvalues) and it is not the theta series of a character of a real quadratic field in which $p$ splits.

(2) It is an Eisenstein series which is irregular at $p$. (In other words, $\varrho_{g}$ is the direct sum of two characters $\chi_{1}$ and $\chi_{2}$ for which $\chi_{1}(p)=\chi_{2}(p)$.)

Thanks to Hypothesis $\mathrm{C}$ and its implication that the inclusions in (6) and (7) are isomorphisms, it becomes possible to adapt the definition of the $p$-adic iterated integrals to define a $\mathbb{C}_{p}$-valued trilinear form

$$
I_{p}^{\prime}: S_{2}(N p)_{L}[f] \times M_{1}(N p, \chi)_{L}^{\vee}\left[g_{\alpha}\right] \times M_{1}(N p, \chi)_{L}[h] \longrightarrow \mathbb{C}_{p}
$$

by the simple rule

$$
I_{p}^{\prime}(\breve{f}, \breve{\gamma}, \breve{h})=\int_{\breve{\gamma}} \breve{f} \cdot \breve{h}:=\breve{\gamma}\left(e_{g_{\alpha}^{*}} e_{\text {ord }}(\breve{F} \cdot \breve{h})\right),
$$

where

$$
e_{g_{\alpha}^{*}}: S_{1}^{\text {oc,ord }}(N, \chi) \longrightarrow S_{1}^{\text {oc,ord }}(N, \chi)\left[\left[g_{\alpha}^{*}\right]\right]=M_{1}(N p, \chi)_{\mathbb{C}_{p}}\left[\left[g_{\alpha}^{*}\right]\right]
$$

is the projector onto the generalized eigenspace attached to $g_{\alpha}^{*}$ for the action of the Hecke algebra $\mathbb{T}_{N}^{(p)}$. Note the crucial role played by Hypothesis $\mathrm{C}$ in the definition of this trilinear form via the equality in (9). 
We are now almost ready to state Conjecture ES relating the trilinear form in (8) to arithmetic data associated to $\left(E, \varrho_{g}, \varrho_{h}\right)$. Since the prime $p$ is unramified in $H$, there is an embedding $H \longrightarrow \mathbb{Q}_{p}^{\text {ur }}$ into the maximal unramified extension of $\mathbb{Q}_{p}$. Fix such an embedding, and denote by $\sigma_{p} \in \mathrm{Gal}(H / \mathbb{Q})$ the associated Frobenius element at $p$. If $F$ is any finite extension of $\mathbb{Q}_{p}$, with ring of integers $\mathcal{O}_{F}$, let

$$
\log _{p}: \mathcal{O}_{F}^{\times} \longrightarrow F
$$

be the usual $p$-adic logarithm, and let

$$
\log _{E, p}: E(F) \longrightarrow F
$$

denote the canonical extension to $E(F)$ of the $p$-adic formal group logarithm associated to a Néron differential on $E$. Via the chosen embedding of $H$ into $\mathbb{C}_{p}$, the maps $\log _{p}$ and $\log _{E, p}$ give rise to homomorphisms

$$
\log _{p}:\left(\mathcal{O}_{H}\right)_{L}^{\times} \longrightarrow H_{p} \otimes L, \quad \log _{E, p}: E(H)_{L} \longrightarrow H_{p} \otimes L .
$$

Hypotheses A and B together imply that $r_{\text {an }}\left(E, \varrho_{g h}\right) \geqslant 2$, and of course the same is expected of the algebraic rank. When $r\left(E, \varrho_{g h}\right)=2$, let $\left(\Phi_{1}, \Phi_{2}\right)$ be an $L$-vector space basis for $\operatorname{Hom}_{G_{\mathbb{Q}}}\left(V_{g h}, E(H)_{L}\right)$.

Using Hypothesis $C^{\prime}$, we make a choice of a one-dimensional $G_{\mathbb{Q}_{p}}$-stable subspace of $V_{g}$, denoted $V_{g}^{g_{\alpha}}$, according to the following recipe.

(1) When condition (1) in Hypothesis $\mathrm{C}^{\prime}$ is satisfied, the Frobenius element $\sigma_{p}$ acts on the Artin representations $V_{g}$ with distinct eigenvalues $\alpha_{g}$ and $\beta_{g}$, and thus determines a decomposition $V_{g}=V_{g}^{g_{\alpha}} \oplus V_{g}^{g \beta}$ into one-dimensional $\sigma_{p}$-eigenspaces with eigenvalue $\alpha_{g}$ and $\beta_{g}$, respectively.

(2) When condition (2) in Hypothesis $\mathrm{C}^{\prime}$ is satisfied, we simply let $V_{g}^{g_{\alpha}}$ be any one-dimensional subspace of the (reducible) representation $V_{g}$ which is not stable under $G_{\mathbb{Q}}$.

The choice of $V_{g}^{g_{\alpha}}$ then determines a canonical two-dimensional subspace of $V_{g h}$ by the rule

$$
V_{g h}^{g_{\alpha}}:=V_{g}^{g_{\alpha}} \otimes V_{h} .
$$

Fix an $L$-vector space basis $\left(v_{1}, v_{2}\right)$ for this space.

The bases $\left(\Phi_{1}, \Phi_{2}\right)$ and $\left(v_{1}, v_{2}\right)$ give rise to a $2 \times 2$ regulator matrix

$$
R_{g_{\alpha}}\left(E, \varrho_{g h}\right):=\left(\begin{array}{ll}
\log _{E, p}\left(\Phi_{1}\left(v_{1}\right)\right) & \log _{E, p}\left(\Phi_{1}\left(v_{2}\right)\right) \\
\log _{E, p}\left(\Phi_{2}\left(v_{1}\right)\right) & \log _{E, p}\left(\Phi_{2}\left(v_{2}\right)\right)
\end{array}\right)
$$


which is independent of the choice of basis $\left(\Phi_{1}, \Phi_{2}\right)$ (respectively, of the basis $\left.\left(v_{1}, v_{2}\right)\right)$ up to left (respectively, right) multiplication by a matrix in $\mathbf{G L}_{2}(L)$. The determinant of $R_{g_{\alpha}}\left(E, \varrho_{g h}\right)$ is therefore well defined, up to multiplication by $L^{\times}$. It can also be shown that this determinant does not depend on the choice of $V_{g}^{g_{\alpha}}$ when Condition (2) of Hypothesis $\mathrm{C}^{\prime}$ is satisfied. In both cases (1) and (2), it belongs to $\mathbb{Q}_{p}^{\text {ur }} \otimes L$, and $\sigma_{p}$ acts on it with the same eigenvalue as on $\wedge^{2} V_{g h}^{g_{\alpha}}$, namely, $\alpha_{g} \alpha_{h} \alpha_{g} \beta_{h}=\alpha_{g} / \beta_{g}$.

Let $\operatorname{Ad}_{g}:=\operatorname{Hom}^{0}\left(V_{g}, V_{g}\right)$ denote the three-dimensional adjoint representation attached to $\varrho_{g}$, on which the Frobenius element $\sigma_{p}$ acts with eigenvalues 1, $\alpha_{g} / \beta_{g}$, and $\beta_{g} / \alpha_{g}$. Section 1.2 attaches to $g_{\alpha}$ a Stark unit $u_{g_{\alpha}} \in\left(\mathcal{O}_{H_{g}}[1 / p]^{\times}\right)_{L}^{\mathrm{Ad}_{g}}$, where $H_{g}$ is the number field cut out by $\operatorname{Ad}_{g}$.

(1) When $g$ satisfies condition (1) in Hypothesis $C^{\prime}$ and $\alpha_{g} / \beta_{g} \neq-1$, this unit is determined up to scaling by the twin requirements that

$$
u_{g_{\alpha}} \text { belongs to }\left(\mathcal{O}_{H_{g}}^{\times}\right)_{L}^{\mathrm{Ad}_{g}}, \quad \sigma_{p}\left(u_{g_{\alpha}}\right)=\frac{\alpha_{g}}{\beta_{g}} u_{g_{\alpha}} .
$$

A more detailed discussion of $u_{g_{\alpha}}$ is given in Section 1.2. The unit $u_{g_{\alpha}}$ is analogous to the Stark units of [St2, DuLi] whose logarithms encode the Petersson scalar product of $g_{\alpha}$ with itself. Section 1.2 explains the striking connection between the existence of $u_{g_{\alpha}}$ and Hypothesis $\mathrm{C}^{\prime}$.

(2) When $g$ satisfies condition (2) in Hypothesis $C^{\prime}$, then

$$
u_{g_{\alpha}} \text { belongs to }\left(\mathcal{O}_{H_{g}}[1 / p]^{\times}\right)_{L}^{\mathrm{Ad}_{g}} \text {. }
$$

Note that in this case $H_{g}$ is the abelian extension cut out by the (odd) Dirichlet character $\chi_{1} / \chi_{2}$, in which the prime $p$ splits completely.

The main conjecture of the present work is the following.

Conjecture ES (Elliptic Stark conjecture). Assume that Hypotheses A, B and $\mathrm{C}-\mathrm{C}^{\prime}$ are satisfied, hence in particular $r_{\mathrm{an}}\left(E, \varrho_{g h}\right) \geqslant 2$. If $r\left(E, \varrho_{g h}\right)>2$, then the trilinear form $I_{p}^{\prime}$ of $(8)$ is identically zero. If $r\left(E, \varrho_{g h}\right)=2$, there exist 'test vectors'

$$
(\breve{f}, \breve{\gamma}, \breve{h}) \in S_{2}(N p)_{L}[f] \times M_{1}(N p, \chi)_{L}^{\vee}\left[g_{\alpha}\right] \times M_{1}(N p, \chi)_{L}[h]
$$

for which

$$
\int_{\breve{\gamma}} \breve{f} \cdot \breve{h}=\frac{\operatorname{det}\left(R_{g_{\alpha}}\left(E, \varrho_{g h}\right)\right)}{\log _{p}\left(u_{g_{\alpha}}\right)} .
$$


Note that both the numerator and denominator on the right-hand side of (10) belong to the same eigenspace for $\sigma_{p}$, with eigenvalue $\alpha_{g} / \beta_{g}$, and hence that the ratio belongs to $L_{p} \subset H_{p} \otimes L$, consistent with the fact that this is clearly true of the left-hand side.

The condition $r\left(E, \varrho_{g h}\right)=2$ implies that either

(1) the Artin representation $\varrho_{g h}$ admits a decomposition

$$
\varrho_{g h}=\varrho_{1} \oplus \varrho_{2} \quad \text { with } r\left(E, \varrho_{1}\right)=2, r\left(E, \varrho_{2}\right)=0,
$$

where $\varrho_{1}$ is an irreducible constituent of $\varrho_{g h}$ and $\varrho_{2}$ is possibly trivial, or reducible; or

(2) the Artin representation $\varrho_{g h}$ admits a decomposition

$$
\varrho_{g h}=\varrho_{1} \oplus \varrho_{2} \oplus \varrho_{3} \quad \text { with } r\left(E, \varrho_{1}\right)=r\left(E, \varrho_{2}\right)=1, r\left(E, \varrho_{3}\right)=0,
$$

where $\varrho_{1}$ and $\varrho_{2}$ are irreducible constituents of $\varrho_{g h}$, and $\varrho_{3}$ is possibly trivial or reducible.

These scenarios will be referred to respectively as the rank-2 and rank-(1, 1) settings for the elliptic Stark conjecture. The apparent rarity of double zeroes for primitive $L$-functions at central critical points suggests that rank-2 settings may only occur sporadically, while a systematic supply of rank- $(1,1)$ settings arises when the constituents $\varrho_{1}$ and $\varrho_{2}$ in (12) are self-dual and the signs in the functional equations for $L\left(E, \varrho_{1}, s\right)$ and $L\left(E, \varrho_{2}, s\right)$ are both equal to -1 . This explains why more of the experiments reported on below have tested Conjecture ES in the rank- $(1,1)$ setting (although enough data has been gathered to lend convincing evidence for Conjecture ES in the rank-2 scenario as well).

Another attractive feature of the rank- $(1,1)$ setting is that it often leads to explicit formulae for the $p$-adic logarithms of global points in $E(H)_{L}^{\varrho_{1}}$ and $E(H)_{L}^{\varrho_{2}}$. More precisely, the regulator $R_{g_{\alpha}}\left(E, \varrho_{g h}\right)$ is said to be factorizable if, after eventually interchanging $\alpha_{h}$ and $\beta_{h}$ and/or $\varrho_{1}$ and $\varrho_{2}$, the Frobenius $\sigma_{p}$ acting on $V_{\varrho_{1}}$ admits $\alpha_{g} \alpha_{h}$ but not $\alpha_{g} \beta_{h}$ as an eigenvalue. Relative to a basis $\left(\Phi_{1}, \Phi_{2}\right) \in \operatorname{Hom}_{G_{\mathbb{Q}}}\left(V_{\varrho_{1}}, E(H)_{L}\right) \times \operatorname{Hom}_{G_{\mathbb{Q}}}\left(V_{\varrho_{2}}, E(H)_{L}\right)$ and to a $\sigma_{p}$-eigenbasis $\left(v_{1}, v_{2}\right)$ for $V_{g h}^{g \alpha}$ satisfying

$$
\sigma_{p} v_{1}=\alpha_{g} \alpha_{h} v_{1}, \quad \sigma_{p} v_{2}=\alpha_{g} \beta_{h} v_{2},
$$

the regulator matrix $R_{g_{\alpha}}$ takes on the simple form

$$
R_{g_{\alpha}}\left(E, \varrho_{g h}\right)=\left(\begin{array}{cc}
\log _{E, p} P_{1} & 0 \\
\log _{E, p} Q & \log _{E, p} P_{2}
\end{array}\right)
$$


where the points $P_{j}$ belong to $E(H)_{L}^{\varrho_{j}}$ and are uniquely determined (up to $L^{\times}$) by the requirement that

$$
\sigma_{p} P_{1}=\alpha_{g} \alpha_{h} P_{1}, \quad \sigma_{p} P_{2}=\alpha_{g} \beta_{h} P_{2} .
$$

Conjecture ES then admits the simpler formulation

$$
\int_{\breve{\gamma}} \breve{f} \cdot \breve{h}=\frac{\log _{E, p}\left(P_{1}\right) \cdot \log _{E, p}\left(P_{2}\right)}{\log _{p}\left(u_{g_{\alpha}}\right)} .
$$

The prototype for such a formula, discovered experimentally, is equation (45) in Remark 3.4.

One of the important motivations for studying Conjecture ES is the connection between the $p$-adic Rankin $L$-values it considers and the generalized Kato classes

$$
\begin{gathered}
\kappa\left(f, g_{\alpha}, h_{\alpha}\right), \quad \kappa\left(f, g_{\alpha}, h_{\beta}\right), \quad \kappa\left(f, g_{\beta}, h_{\alpha}\right), \\
\kappa\left(f, g_{\beta}, h_{\beta}\right) \in H^{1}\left(\mathbb{Q}, V_{p}(E) \otimes V_{g h}\right)
\end{gathered}
$$

constructed in [DR2] (respectively, in [BDR2]) as $p$-adic limits of étale AbelJacobi images of Gross-Kudla-Schoen diagonal cycles (respectively, of étale regulators of Beilinson-Kato and/or Beilinson-Flach elements) when $g$ and $h$ are cusp forms (respectively, when $g$ and/or $h$ are Eisenstein series). When $L\left(E, \varrho_{g h}, 1\right)=0$, these generalized Kato classes can be viewed as playing the role of Heegner points in settings of (analytic) rank two; the reader is referred to Theorems B and D and Section 6.3 of [DR2] for the relationship between their $p$-adic logarithms and the $p$-adic iterated integrals attached to $(f, g, h)$. The calculations performed in this work have also played a crucial role in the more recent preprint [DR3], which conjectures a precise expression for the generalized Kato classes as linear combinations of points in the relevant Mordell-Weil group.

Turning to a more detailed overview of the contents of the paper, Section 1 discusses Hypothesis $\mathrm{C}$ and defines the Gross-Stark unit $u_{g_{\alpha}}$, showing that Hypothesis $\mathrm{C}$ bears an intimate connection with the existence of this unit.

Building on the results of Harris and Kudla, and of Ichino, on trilinear periods and Jacquet's conjecture, Section 2 recasts the trilinear form $I_{p}^{\prime}$ as the value of a Garrett-Hida triple-product $p$-adic $L$-function at a point lying outside its region of classical interpolation. This connection with $p$-adic $L$-functions justifies viewing Conjecture ES as an elliptic curve analogue of the ( $p$-adic) Stark conjectures. It also suggests that Conjecture ES might be regarded as a $p$-adic limit of Jacquet's conjecture on trilinear periods, prompting a possible strategy for proving it in some cases and suggesting that it might be viewed as a deep manifestation of the 'Artin formalism' for certain $p$-adic $L$-series. 
The rest of the paper is devoted to giving both theoretical and experimental evidence in support of Conjecture ES.

Sections 3 and 4 treat the case where $\varrho_{g}$ and $\varrho_{h}$ are induced from characters $\psi_{g}$ and $\psi_{h}$ of the same quadratic field $K$, and with inverse central characters. The representation

$$
V_{g h}=V_{\psi_{1}} \oplus V_{\psi_{2}}
$$

then decomposes as the direct sum of a pair of representations induced from ring class characters $\psi_{1}$ and $\psi_{2}$ of $K$.

Section 3 focusses on the setting where $K$ is imaginary quadratic. Building on the results of Section 2 and the precise formulae of Watson [Wa] and Woodbury [Wo1], Conjecture ES is proved in Section 3.2 under the assumption that the prime $p$ splits in $K$ and a Heegner hypothesis is satisfied. The main ingredients in the proof are the $p$-adic Gross-Zagier formula of [BDP2] expressing Heegner points in terms of values of a Katz-style $p$-adic Rankin $L$-series, the relation established in [DR1] between $p$-adic iterated integrals and Garrett-Hida $p$-adic $L$-series, and a subtle relation between these two types of $L$-series. It is in the nontrivial comparison between periods used in their definitions that a value of Katz's two-variable $p$-adic $L$-function makes an appearance. The unit $u_{g_{\alpha}}$ can be expressed in terms of elliptic units in this case, and emerges from Katz's $p$-adic analogue of the Kronecker limit formula.

Katz's approach to $p$-adic $L$-functions (as well as the closely related approach of [BDP2]) is based on the evaluation of $p$-adic modular forms at complex multiplication (CM) points when they lie on the ordinary locus of the modular curve, that is, when $p$ is split in $K$. This approach breaks down entirely when $p$ is inert in the quadratic imaginary field. Section 3.3 gives numerical evidence in support of Conjecture ES in the inert case, both in the rank- $(1,1)$ setting-where the theory of elliptic units and Heegner points can still be used to produce the global objects that should enter into the right-hand side of (10) — and in the more mysterious rank-2 setting.

Section 4 attempts a parallel treatment when $\varrho_{g}$ and $\varrho_{h}$ are induced from characters (of mixed signature) of the same real quadratic field $K$. In some cases, the global points $P_{1}$ and $P_{2}$ can then be expressed, at least conjecturally, in terms of the Stark-Heegner points of [Dar] and [Gre] attached to 'real quadratic cycles on $\mathcal{H}_{p} \times \mathcal{H}^{\prime}$ '. The resulting relation between the Stark points of the present work and these Stark-Heegner points might be amenable to proof via the methods of Section 3.2. The prospect of tying the real quadratic cycles on $\mathcal{H}_{p} \times \mathcal{H}$ to global (but not necessarily motivic) cohomology classes in the Selmer groups of $E$ over ring class fields of real quadratic fields represents an encouraging first step towards better understanding their global nature. 
Sections 5-7 consider the remaining interesting cases, all of which appear to lie beyond the reach of previously available theory or conjectural construction.

Section 5 reports on a number of experiments in the setting where $h$ is an exotic weight-one form, whose associated Galois representation has projective image $A_{4}, S_{4}$, or $A_{5}$, and $g=h^{*}$ is its dual. The representation $V_{g h}$ then breaks up as

$$
V_{g h}=L \oplus \operatorname{Ad}_{g}
$$

In the rank- $(1,1)$ setting, Conjecture ES leads to a $p$-adic analytic construction of a global point on $E$ over the field cut out by $\operatorname{Ad}_{g}$. The setting where the Hasse-Weil $L$-series $L(E, s)$ has a double zero at the centre and $L\left(E, \operatorname{Ad}_{g}, 1\right)$ $\neq 0$ is particularly interesting: Conjecture ES then predicts the vanishing of the $p$-adic iterated integrals, even though the generalized Kato class $\kappa\left(f, g_{\alpha}, \bar{g}_{\bar{\alpha}}\right)$ is expected to be a canonical, nontrivial element of the pro- $p$ Selmer group of $E / \mathbb{Q}$ : we refer to [DR3] for a fuller discussion of this setting.

Section 6 takes up the setting where $g \in S_{1}(N, \bar{\chi})$ is a cusp form and $h:=$ $E_{1}(1, \chi) \in M_{1}(N, \chi)$ is the weight-one Eisenstein series attached to the pair $(1, \chi)$ of Dirichlet characters. A novelty of this setting is that

$$
V_{g h}=V_{g} \oplus V_{g^{*}}
$$

decomposes into a direct sum of two representations which are not self-dual in general, and the regulator matrix $R_{g_{\alpha}}\left(E, \varrho_{g h}\right)$ involves points in the isotypic parts for these non self-dual representations.

Section 7 concludes with the setting in which $g:=E_{1}\left(1, \chi^{-1}\right)$ is an (irregular) Eisenstein series. The case where $h:=E_{1}\left(\chi_{1}, \chi_{2}\right)$ is also an Eisenstein series (with $\chi_{1} \chi_{2}=\chi$ ) is particularly appealing, since

$$
V_{g h}=\chi_{1} \oplus \bar{\chi}_{1} \oplus \chi_{2} \oplus \bar{\chi}_{2}
$$

is then a direct sum of one-dimensional representations. In this setting-the most 'degenerate instance' of the one described in Section 6-the relevant 'generalized Kato classes' coincide with the classes constructed by Kato from $p$-adic families of Beilinson elements, whose behaviour has a bearing on the arithmetic of elliptic curves over cyclotomic fields. Section 7 reports on experimental evidence in support of Conjecture ES, where the regulators attached to $\left(f, g_{\alpha}, h_{\alpha}\right)$ involve logarithms of points on $E$ defined over the abelian extension of $\mathbb{Q}$ cut out by either $\chi_{1}$ or $\chi_{2}$. The scenario in which these Dirichlet characters are nonquadratic is particularly intriguing. According to predictions of David et al. [DFK], the occurrence of global points over cyclic extensions of degree greater than two is quite sporadic; the $L$-series that control them have 
nonreal coefficients, and their functional equation gives no indication as to the parity of their order of vanishing at the centre. Although ostensibly rare, global points over cyclic cubic extensions do occur in practice. Fairly extensive tables of pairs $(E, \chi)$ with $\chi$ cubic for which $r_{\mathrm{an}}(E, \chi)>0$ have been drawn up by the authors of $[\mathrm{DFK}]$, and the computationally more challenging task of verifying that $r(E, \chi)>0$ for such pairs is carried out-albeit in more limited ranges-in [Co]. Section 7.2 describes a few experimental verifications of Conjecture ES involving the $p$-adic logarithms of such cyclic cubic points.

The article concludes with a table summarizing the main features of the numerical experiments described in this work.

\section{Overconvergent weight-one forms and Gross-Stark units}

1.1. Classical and overconvergent modular forms of weight one. One of the main results of this section is the following concrete criterion, following directly from a result of Cho and Vatsal and of Bellaïche and Dimitrov, which shows that Hypothesis $\mathrm{C}$ is frequently satisfied in practice.

Proposition 1.1. Let $g \in S_{1}\left(N, \chi^{-1}\right)$ be a cusp form of weight one which is regular at $p$, and let $g_{\alpha}$ denote one of its p-stabilizations. Then the natural inclusion

$$
S_{1}(N p, \chi)_{\mathbb{C}_{p}}\left[g_{\alpha}^{*}\right] \hookrightarrow S_{1}^{\mathrm{oc}, \text { ord }}(N, \chi)\left[\left[g_{\alpha}^{*}\right]\right]
$$

is an isomorphism of $\mathbb{C}_{p}$-vector spaces if and only if

$\varrho_{g}$ is not induced from a character of a real quadratic field in which $p$ splits.

Proof. Let $\mathbb{T}_{k}(N)$ denote the $\mathbb{Z}_{p}$-algebra generated by the Hecke operators $T_{\ell}$ (with $\ell \nmid N$ ) and $U_{q}$ (with $q \mid N$ ), together with the diamond operators, acting on the space $M_{k}(N)$ of classical modular forms on $\Gamma_{1}(N)$. When $k \geqslant 2$, there is a natural perfect duality

$$
\mathbb{T}_{k}(N) \times M_{k}(N) \longrightarrow \mathbb{Z}_{p}
$$

defined by $\langle T, f\rangle:=a_{1}(T f)$.

Hida's $\Lambda$-adic Hecke algebra is defined to be

$$
\underline{\mathbb{T}}(N)=\lim _{\leftarrow, t}\left(e_{\text {ord }} \mathbb{T}_{2}\left(N p^{t}\right)\right) \otimes_{\mathbb{Z}_{p}} \mathbb{Q}_{p},
$$

where the inverse limit is taken with respect to the natural projection maps. The algebra $\underline{\mathbb{T}}(N)$ has a natural structure of a $\Lambda \otimes_{\mathbb{Z}_{p}} \mathbb{Q}_{p}$-module arising from the 
action of the diamond operators, and it can also be viewed as a module over the $\Lambda \otimes \mathbb{Q}_{p}$-subalgebra $\underline{\mathbb{T}}^{0}(N)$ of $\underline{\mathbb{T}}(N)$ generated by the 'good' Hecke operators $T_{n}$ with $(n, p N)=1$ and by $U_{p}$. Let $\varphi_{k}: \Lambda \otimes \mathbb{Q}_{p} \longrightarrow \mathbb{Q}_{p}$ denote the 'weight- $k$ specialization map' sending a group-like element $\langle a\rangle \in 1+p \mathbb{Z}_{p} \subset \Lambda^{\times}$to $a^{k-1}$, and let $\wp_{k}$ denote its kernel. The pairing of (15) induces a perfect duality

$$
\left(\left(\underline{\mathbb{T}}(N) / \wp_{k}\right) \otimes_{\mathbb{Q}_{p}} \mathbb{C}_{p}\right) \times M_{k}^{\mathrm{oc}, \text { ord }}(N) \longrightarrow \mathbb{C}_{p}
$$

In particular, the space $M_{1}^{\text {oc, ord }}(N)$ is naturally identified with the vector space of $\mathbb{C}_{p}$-linear homomorphisms $\operatorname{Hom}\left(\underline{\mathbb{T}}(N) / \wp_{1}, \mathbb{C}_{p}\right)$. Under this identification, the classical weight-one form $g_{\alpha} \in M_{1}^{\text {oc,ord }}(N)$, which is a normalized eigenvector for the good Hecke operators $T_{n}$ with $(n, N p)=1$ and for $U_{p}$, corresponds to an algebra homomorphism

$$
\varphi_{g_{\alpha}} \in \operatorname{Hom}_{\mathrm{alg}}\left(\underline{\mathbb{T}}^{0}(N) / \wp_{1}, \mathbb{C}_{p}\right) .
$$

Let $\wp_{g_{\alpha}} \subset \underline{T}^{0}(N)$ be the kernel of $\varphi_{g_{\alpha}}$. Its image under the 'projection map to weight space'

$$
\operatorname{Hom}_{\text {alg }}\left(\underline{\mathbb{T}}^{0}(N), \mathbb{C}_{p}\right) \longrightarrow \operatorname{Hom}_{\mathrm{alg}}\left(\Lambda, \mathbb{C}_{p}\right)
$$

induced by restriction is equal to $\varphi_{1}$, that is, $\wp_{g_{\alpha}}$ contains the ideal $\wp_{1} \underline{\mathbb{T}}^{0}(N)$.

The eigenspace $M_{1}^{\text {oc,ord }}(N)\left[g_{\alpha}^{*}\right]$ that arises in the statement of Proposition 1.1 can be identified with the dual of $\underline{\mathbb{T}}(N) / \wp_{g_{\alpha}}$ :

$$
M_{1}^{\mathrm{oc}, \text { ord }}(N)\left[g_{\alpha}^{*}\right]=\operatorname{Hom}\left(\underline{\mathbb{T}}(N) / \wp_{g_{\alpha}}, \mathbb{C}_{p}\right) .
$$

Furthermore,

$$
M_{1}^{\mathrm{oc}, \text { ord }}(N)\left[\left[g_{\alpha}^{*}\right]\right]=\operatorname{Hom}\left(\underline{\mathbb{T}}(N)_{\wp_{g \alpha}} / \wp_{1}, \mathbb{C}_{p}\right),
$$

where

$$
\underline{\mathbb{T}}^{0}(N)_{\wp_{g \alpha}}:=\lim _{\leftarrow, n} \mathbb{T}^{0}(N) / \wp_{g_{\alpha}}^{n}, \quad \underline{\mathbb{T}}(N)_{\wp_{g \alpha}}:=\lim _{\leftarrow, n} \mathbb{T}(N) / \wp_{g_{\alpha}}^{n},
$$

denote the completions at the maximal ideal $\wp_{g_{\alpha}}$ of $\underline{\mathbb{T}}^{0}(N) \otimes \mathbb{C}_{p}$.

Hence the dual of the natural inclusion $M_{1}^{\text {oc,ord }}(N)\left[g_{\alpha}^{*}\right] \hookrightarrow M_{1}^{\text {oc,ord }}(N)\left[\left[g_{\alpha}^{*}\right]\right]$ is the natural surjection

$$
\underline{\mathbb{T}}(N)_{\wp_{g \alpha}} / \wp_{1} \longrightarrow \underline{\mathbb{T}}(N) / \wp_{g_{\alpha}} .
$$


The ideal $\wp_{1} \underline{\mathbb{T}}^{0}(N)_{\wp_{g_{\alpha}}}$ is equal to a positive power $\wp_{g_{\alpha}}^{t}$ (with $t \geqslant 1$ ) of the maximal ideal $\wp_{g_{\alpha}}$ in the local ring $\underline{\mathbb{T}}^{0}(N)_{\wp_{g_{\alpha}}} \otimes \mathbb{C}_{p}$. The map (16) is an isomorphism if and only if $t=1$, that is, if and only if the point of $\operatorname{Hom}\left(\underline{\mathbb{T}}^{0}(N)_{\wp_{g}}, \mathbb{C}_{p}\right)$ attached to $\varphi_{g_{\alpha}}$ is smooth and étale over weight space. Under the regularity assumption on $g$ at $p$, [BeDi, Theorem 1.1] implies that this étaleness condition is satisfied precisely when (14) holds. The proposition follows.

REMARK 1.2. When $g$ is the theta series of a real quadratic field in which $p$ splits, explicit Fourier expansions of nonclassical forms in $S_{1}^{\text {oc,ord }}(N, \chi)\left[\left[g_{\alpha}^{*}\right]\right]$ are described in [DLR2]. Such nonclassical forms are also expected to be present in all settings where $g$ is irregular at $p$ : see [DLR3] for a study of the nonclassical forms in the associated generalized eigenspace in this case. Hypothesis $\mathrm{C}$ and condition (1) of Hypothesis $\mathrm{C}^{\prime}$ are thus expected to be equivalent when $g$ is a cusp form. The extension of Conjecture ES to settings where $S_{1}^{\text {oc,ord }}(N, \chi)_{\mathbb{C}_{p}}\left[\left[g_{\alpha}^{*}\right]\right]$ contains nonclassical forms, which is far from routine and involves significant new features, is taken up in [DLR3].

We next turn to the case where $g=E_{1}\left(\chi_{1}, \chi_{2}\right)$ is a weight-one Eisenstein series with $\alpha_{g}:=\chi_{1}(p)$ and $\beta_{g}:=\chi_{2}(p)$, and let

$$
g_{\alpha}(q):=g(q)-\chi_{2}(p) g\left(q^{p}\right), \quad g_{\beta}(q):=g(q)-\chi_{1}(p) g\left(q^{p}\right)
$$

denote its (not necessarily distinct) $p$-stabilizations.

Proposition 1.3. The cuspidal generalized eigenspace $S_{1}^{\mathrm{oc}, \text { ord }}(N, \chi)\left[\left[g_{\alpha}^{*}\right]\right]$ is nontrivial, that is, the eigenform $g_{\alpha}$ is a p-adic cusp form, if and only if $\chi_{1}(p)=$ $\chi_{2}(p)$.

Proof. Assume for simplicity that $\chi_{1}$ and $\chi_{2}$ are primitive with relatively prime conductors $N_{1}$ and $N_{2}$, so that $\chi:=\chi_{1} \chi_{2}$ is primitive and $N=N_{1} N_{2}$. We prove the proposition by analysing the constant terms of $g_{\alpha}$ and $g_{\beta}$ at the cusps in the ordinary locus of the modular curve $X(N ; p)=X_{1}(N) \times_{X(1)} X_{0}(p)$ associated to the congruence group $\Gamma_{1}(N) \cap \Gamma_{0}(p)$. More precisely, let $X_{1}(N)_{\mathbb{C}_{p}}^{\text {ord }}$ denote the complement of the supersingular residue discs in $X_{1}(N)\left(\mathbb{C}_{p}\right)$, regarded as an affinoid domain. For a suitable wide open neighbourhood $\mathcal{W}$ of $X_{1}(N)_{\mathbb{C}_{p}}^{\text {ord }}$, let

$$
s: \mathcal{W} \longrightarrow X(N ; p)_{\mathbb{C}_{p}}
$$

be the rigid analytic section of the natural projection $X(N ; p) \longrightarrow X_{1}(N)$, defined by sending the point attached to an ordinary pair $\left(E, P_{N}\right)_{/ \mathbb{C}_{p}} \in \mathcal{W}$ to 
the (moduli of the) triple $\left(E, P_{N}, C_{\text {can }}\right)$, where $P_{N}$ is a point of order $N$ on $E$, and $C_{\text {can }}$ denotes the canonical subgroup of order $p$. The inclusion $i_{g_{\alpha}}$ of (7) in the Introduction is described geometrically in terms of $s$, as follows: letting $\underline{\omega}$ denote the sheaf over $X(N ; p)$ with logarithmic poles at the cusps discussed in [BDP2, Section 1.1], a classical weight-one form $\phi$ in the source of (7) may be viewed as a section of $\underline{\omega}$ over $X(N ; p)$, and the map of (7) sends it to its pullback $s^{*}(\phi) \in H^{0}(\mathcal{W}, \underline{\omega})$, naturally identified with an overconvergent modular form of level $N$.

The nonzero constant terms of the classical form $g=E_{1}\left(\chi_{1}, \chi_{2}\right)$ are concentrated at the cusps of $X_{1}(N)$ attached to the 'Katz test objects'

$$
c_{a b}:=\left(\mathbb{G}_{m} / q^{\mathbb{Z}}, \zeta_{N_{1}}^{a} q^{b / N_{2}}\right), \quad d_{a b}:=\left(\mathbb{G}_{m} / q^{\mathbb{Z}}, q^{a / N_{1}} \zeta_{N_{2}}^{b}\right),
$$

where $\mathbb{G}_{m} / q^{\mathbb{Z}}$ is the Tate generalized elliptic curve over $\operatorname{Spec}\left(\mathbb{C}_{p}[[q]]\right)$, and $\zeta_{N_{1}}$ and $\zeta_{N_{2}}$ are fixed primitive $N_{1}$ and $N_{2}$ th roots of unity; the indices $(a, b)$ range over $\left(\mathbb{Z} / N_{1} \mathbb{Z}\right)^{\times} \times\left(\mathbb{Z} / N_{2} \mathbb{Z}\right)^{\times}$. If $g$ is any weight-one modular form on $\Gamma_{0}(N)$ with character $\chi$, then the modular form $g_{p}$ on $X(N ; p)$ whose $q$-expansion is equal to $g\left(q^{p}\right)$ at the cusp $\infty=\left(\mathbb{G}_{m} / q^{\mathbb{Z}}, \zeta_{N}, \mu_{p}\right)$ is defined on test objects of the form $\left(E, P_{N}, C_{p}\right)$, with $C_{p} \subset E$ of order $p$, by the rule

$$
g_{p}\left(\left(E, P_{N}, C_{p}\right)\right):=\chi^{-1}(p) p^{-1} \varphi_{C_{p}}^{*}\left(g\left(E / C_{p}, P_{N}+C_{p}\right)\right),
$$

where $\varphi_{C_{p}}: E \longrightarrow E / C_{p}$ is the natural quotient morphism. This can be seen by checking this identity on the test object $\left(\mathbb{G}_{m} / q^{\mathbb{Z}}, \zeta_{N}, \mu_{p}\right)$ and invoking the $q$ expansion principle. Let $g_{a b}(q)$ and $g_{a b}^{\prime}(q) \in \mathbb{C}_{p}\left[\left[q^{1 / N}\right]\right]$ denote the $q$-expansions of $g$ at the cusps $c_{a b}$ and $d_{a b}$, respectively, and let $x_{a b}$ and $y_{a b}$ denote the associated leading terms. The $q$-expansion of $g_{p}$, viewed as a $p$-adic modular form, at the cusps $c_{a b}$ and $d_{a b}$ is then given by the rule

$$
\begin{aligned}
g_{p}\left(\mathbb{G}_{m} / q^{\mathbb{Z}}, \zeta_{N_{1}}^{a} q^{b / N_{2}}\right) & =\chi^{-1}(p) p^{-1} \varphi^{*}\left(g\left(\mathbb{G}_{m} / q^{p \mathbb{Z}}, \zeta_{N_{1}}^{p a} q^{p b / N_{2}}\right)\right) \\
& =\chi_{2}^{-1}(p) g_{a b}\left(q^{p}\right) \frac{d t}{t} \\
g_{p}\left(\mathbb{G}_{m} / q^{\mathbb{Z}}, q^{a / N_{1}} \zeta_{N_{2}}^{b}\right) & =\chi^{-1}(p) p^{-1} \varphi^{*}\left(g\left(\mathbb{G}_{m} / q^{p \mathbb{Z}}, q^{p a / N_{1}} \zeta_{N_{2}}^{p b}\right)\right) \\
& =\chi_{1}^{-1}(p) g_{a b}^{\prime}\left(q^{p}\right) \frac{d t}{t} .
\end{aligned}
$$

Here, we have used the fact the canonical subgroup $\mu_{p} \subset \mathbb{G}_{m} / q^{\mathbb{Z}}$ of the Tate elliptic curve is the kernel of the map $\varphi: \mathbb{G}_{m} / q^{\mathbb{Z}} \longrightarrow \mathbb{G}_{m} / q^{p \mathbb{Z}}$ sending $t$ to $t^{p}$. Equations (17) and (18) yield the constant terms of the forms $g, g_{p}, g_{\alpha}$, and $g_{\beta}$ 
at the cusps $c_{a b}$ and $d_{a b}$, as summarized in the table below.

\begin{tabular}{ccc}
\hline & $c_{a b}$ & $d_{a b}$ \\
\hline$g$ & $x_{a b}$ & $y_{a b}$ \\
$g_{p}$ & $\chi_{2}^{-1}(p) x_{a b}$ & $\chi_{1}^{-1}(p) y_{a b}$ \\
$g_{\alpha}$ & 0 & $\left(1-\chi_{2} / \chi_{1}(p)\right) y_{a b}$ \\
$g_{\beta}$ & $\left(1-\chi_{1} / \chi_{2}(p)\right) x_{a b}$ & 0 \\
\hline
\end{tabular}

The proposition follows.

REMARK 1.4. If $g=E_{1}\left(\chi_{1}, \chi_{2}\right)$ is regular, then the fact that $S_{1}^{\text {oc,ord }}(N, \chi)$ $\left[\left[g_{\alpha}^{*}\right]\right]=0$ shows that the $p$-adic iterated integrals attached to $\left(f, g_{\alpha}, h_{\alpha}\right)$ vanish identically, since the overconvergent weight-one forms $e_{\text {ord }}\left(d^{-1} \breve{f} \times \breve{h}\right)$ belong to $S_{1}^{\text {oc,ord }}(N, \chi)$. It is not hard to check that the regulator term $\operatorname{det}\left(R_{g_{\alpha}}\left(E, \varrho_{g h}\right)\right)$ attached to the subspace $V_{g}^{g_{\alpha}}=\mathbb{C}_{p}\left(\chi_{1}\right)$ vanishes as well, in harmony with Conjecture ES, which can be seen as amounting to the statement $0=0$ when $g$ is a regular Eisenstein series.

1.2. The Gross-Stark unit attached to a weight-one form. The aim of this section is to associate to a $p$-stabilized eigenform $g_{\alpha}$ satisfying Hypothesis $\mathrm{C}^{\prime}$ a global element $u_{g_{\alpha}} \in H^{\times} \otimes L$ (often well defined up to scaling by $L^{\times}$), whose $p$ adic logarithm arises in Conjecture ES. This element belongs to the $\mathrm{Ad}_{g}$-isotypic subspace of $H^{\times} \otimes L$, where

$$
\operatorname{Ad}_{g}:=\operatorname{End}_{0}\left(V_{g}\right)
$$

is the adjoint representation attached to $V_{g}$, the three-dimensional Artin representation consisting of trace-zero $L$-linear endomorphisms of $V_{g}$ on which $G_{\mathbb{Q}}$ acts by conjugation via $\varrho_{g}$.

In defining $u_{g_{\alpha}}$, it shall be convenient to replace the field $H$ of the introduction by the smaller field $H_{g}$ cut out by $\mathrm{Ad}_{g}$, and $L$ by any field over which the representation $\operatorname{Ad}_{g}$ can be defined. To give a rough feeling for the nature of $u_{g_{\alpha}}$, let us mention at the outset that

$$
L \cdot u_{g_{\alpha}} \subset \begin{cases}\left(\mathcal{O}_{H_{g}}^{\times}\right)_{L}^{\mathrm{Ad}_{g}} & \text { if } g \text { is cuspidal, } \\ \left(\mathcal{O}_{H_{g}}[1 / p]^{\times}\right)_{L}^{\mathrm{Ad}_{g}} & \text { if } g \text { is Eisenstein }\end{cases}
$$

and

the Frobenius element $\sigma_{p}$ acts on $u_{g_{\alpha}}$ with eigenvalue $\alpha_{g} / \beta_{g}$.

As explained below, these requirements often characterize $u_{g_{\alpha}}$ uniquely up to scaling by $L^{\times}$. 
Write $\sigma_{\infty} \in G:=\operatorname{Gal}\left(H_{g} / \mathbb{Q}\right)$ for the involution given by complex conjugation induced by the fixed choice of complex embedding of $H_{g} \subset \overline{\mathbb{Q}}$. The representation $\operatorname{Ad}_{g}$ decomposes as a direct sum of + and - eigenspaces for the action of $\sigma_{\infty}$, denoted $\mathrm{Ad}_{g}^{+}$and $\mathrm{Ad}_{g}^{-}$. These eigenspaces are of dimensions one and two, respectively:

$$
\operatorname{dim}_{L} \operatorname{Ad}_{g}^{+}=1 ; \quad \operatorname{dim}_{L} \operatorname{Ad}_{g}^{-}=2 .
$$

As suggested by (19), the nature of the invariant $u_{g_{\alpha}}$ depends on whether $\varrho_{g}$ is irreducible or not, and each case will be dealt with in turn.

1.2.1. The case where $\varrho_{g}$ is irreducible. If $\varrho_{g}$ is irreducible, then $g$ is a cuspidal eigenform of weight one. The definition of $u_{g_{\alpha}}$ rests on the following proposition.

\section{PROPOSITION 1.5.}

(1) The vector space $\operatorname{Hom}_{G_{\mathbb{Q}}}\left(\operatorname{Ad}_{g}, \mathcal{O}_{H_{g}}^{\times} \otimes L\right)$ is one dimensional.

(2) The Artin L-function $L\left(\operatorname{Ad}_{g}, s\right)$ has a simple zero at $s=0$.

Proof. The finite group $G=\operatorname{Gal}\left(H_{g} / \mathbb{Q}\right)$ acts faithfully on $\operatorname{Ad}_{g}$ and is identified with the image of the projective representation attached to $V_{g}$. Note that $G_{\infty}=\left\langle\sigma_{\infty}\right\rangle$ is of order two, since $\operatorname{Ad}_{g}$ is not totally even. Dirichlet's unit theorem determines the structure of $\mathcal{O}_{H_{g}}^{\times} \otimes L$ as an $L[G]$-module:

$$
\mathcal{O}_{H_{g}}^{\times} \otimes L=\left(\operatorname{Ind}_{G_{\infty}}^{G} L\right)-L,
$$

where the two occurrences of $L$ on the right refer to trivial representations (of $G_{\infty}$ and $G$, respectively). The irreducibility of $V_{g}$ implies that the representation $\operatorname{Ad}_{g}$ contains no $G$-invariant vectors, by Schur's lemma, and hence

$$
\operatorname{Hom}_{G}\left(\operatorname{Ad}_{g}, \mathcal{O}_{H_{g}}^{\times} \otimes L\right)=\operatorname{Hom}_{G}\left(\operatorname{Ad}_{g}, \operatorname{Ind}_{G_{\infty}}^{G} L\right) .
$$

By Frobenius reciprocity,

$$
\operatorname{Hom}_{G}\left(\operatorname{Ad}_{g}, \operatorname{Ind}_{G_{\infty}}^{G} L\right)=\operatorname{Hom}_{G_{\infty}}\left(\operatorname{Ad}_{g}, L\right)=\operatorname{Hom}\left(\operatorname{Ad}_{g}^{+}, L\right) .
$$

The first part of the proposition now follows from (20). The second statement follows from the shape of the $\Gamma$-factors in the functional equation of $L\left(\operatorname{Ad}_{g}, s\right)$ determined by (20).

Choose a basis $\varphi$ for the one-dimensional vector space $\operatorname{Hom}_{G}\left(\operatorname{Ad}_{g}, \mathcal{O}_{H_{g}}^{\times} \otimes L\right)$, and let

$$
\left(\mathcal{O}_{H_{g}}^{\times}\right)_{L}^{\mathrm{Ad}_{g}}:=\varphi\left(\operatorname{Ad}_{g}\right) \subset \mathcal{O}_{H_{g}}^{\times} \otimes L .
$$


The $L[G]$-module $\left(\mathcal{O}_{H_{g}}^{\times}\right)_{L}^{\mathrm{Ad}_{g}}$ is of dimension $\leqslant 3$ over $L$ and is abstractly isomorphic to the unique irreducible subrepresentation of $\operatorname{Ad}_{g}$ containing $\operatorname{Ad}_{g}^{+}$.

Recall the prime number $p$ chosen in the introduction. The Frobenius element $\sigma_{p}$ acts on $\operatorname{Ad}_{g}$ with the (not necessarily different) eigenvalues $1, \alpha_{g} / \beta_{g}$, and $\beta_{g} / \alpha_{g}$. Define

$$
U_{g_{\alpha}}:=\left\{u \in\left(\mathcal{O}_{H_{g}}^{\times}\right)_{L}^{\operatorname{Ad}_{g}} \text { such that } \sigma_{p}(u)=\frac{\alpha_{g}}{\beta_{g}} u\right\} .
$$

LEMMA 1.6. Assume that $V_{g}$ is regular. If $\varrho_{g}$ is induced from a character of a real quadratic field in which $p$ splits, then $U_{g_{\alpha}}=0$. Otherwise, $\operatorname{dim}_{L}\left(U_{g_{\alpha}}\right) \geqslant 1$, with equality if either

(1) $\alpha_{g} \neq-\beta_{g}$, that is, $\operatorname{Ad}_{g}$ is regular, or

(2) $\varrho_{g}$ is induced from a character of a quadratic field in which $p$ is inert.

Proof. This lemma is proved by a case by case analysis, according to the following possibilities for $V_{g}$.

(a) $V_{g}$ has imaginary dihedral projective image.

(b) $V_{g}$ has real dihedral projective image.

(c) $g$ is an exotic weight-one form with projective image $A_{4}, S_{4}$, or $A_{5}$.

(a) Imaginary dihedral forms. Assume that the representation $V_{g}$ is induced from a character $\psi_{g}$ of an imaginary quadratic field $K$. Then

$$
\operatorname{Ad}_{g}=L\left(\chi_{K}\right) \oplus V_{\psi},
$$

where $\chi_{K}$ is the quadratic Dirichlet character associated to $K$, and $V_{\psi}:=\operatorname{Ind}_{K}^{\mathbb{Q}} \psi$ is the two-dimensional representation induced from the ring class character $\psi:=$ $\psi_{g} / \psi_{g}^{\prime}$ of $K$. Since $\sigma_{\infty}$ acts with eigenvalue -1 on the first factor $L\left(\chi_{K}\right)$, it follows that $\left(\mathcal{O}_{H_{g}}^{\times}\right)_{L}^{\mathrm{Ad}_{g}}$ is a subrepresentation of $V_{\psi}$.

If $V_{\psi}$ is irreducible, then

$$
\left(\mathcal{O}_{H_{g}}^{\times}\right)_{L}^{\mathrm{Ad}_{g}} \simeq V_{\psi} .
$$

The eigenvalues of $\sigma_{p}$ on $V_{\psi}$ are $\alpha_{g} / \beta_{g}$ and $\beta_{g} / \alpha_{g}$ if $\chi_{K}(p)=1$, and 1 and $-1=\alpha_{g} / \beta_{g}$ if $\chi_{K}(p)=-1$. In either case, $\operatorname{dim}_{L}\left(U_{g_{\alpha}}\right) \geqslant 1$, with equality if either $\alpha_{g} / \beta_{g} \neq-1$ or if $p$ is inert in $K$.

If $V_{\psi}$ is reducible, the character $\psi$ is necessarily quadratic, and $V_{\psi}$ further breaks up as a sum of one-dimensional representations attached to odd and even 
Dirichlet quadratic characters $\chi^{-}$and $\chi^{+}$, respectively, satisfying $\chi^{+} \chi^{-}=\chi_{K}$. Because of this,

$$
\left(\mathcal{O}_{H_{g}}^{\times}\right)_{L}^{\mathrm{Ad}_{g}}=\left(\mathcal{O}_{H_{g}}^{\times}\right)_{L}^{\chi^{+}} \simeq L\left(\chi^{+}\right)
$$

is isomorphic to the one-dimensional representation associated to $\chi^{+}$. The space $U_{g_{\alpha}} \subset\left(\mathcal{O}_{H_{g}}^{\times}\right)_{L}^{\chi^{+}}$is therefore of dimension at most one, with equality if and only if $\alpha_{g} / \beta_{g}=\chi^{+}(p)$. This equality always holds when $p=\wp \wp$ splits in $K$, since in that case

$$
\alpha_{g} / \beta_{g}=\psi(\wp)=\psi(\bar{\wp})=\chi^{+}(p)=\chi^{-}(p) .
$$

(Note that the regularity assumption $\alpha_{g} \neq \beta_{g}$ that is made on $g$ forces all these expressions to be equal to -1 rather than 1.) If $p$ is inert in $K$, then $\sigma_{p}$ acts on $V_{\psi}$ with eigenvalues 1 and -1 , and hence $\alpha_{g} / \beta_{g}=\beta_{g} / \alpha_{g}=-1$. In that case, either $\chi^{+}(p)=-1$ and $\operatorname{dim}\left(U_{g_{\alpha}}\right)=1$, or $p$ splits in the real quadratic field $K^{+}$cut out by $\chi^{+}$, and $U_{g_{\alpha}}=0$. In this last scenario, the representations $V_{\psi}$ and $V_{g}$ are also induced from a character of the real quadratic field $K^{+}$, in which $p$ splits.

(b) Real dihedral forms. If the representation $V_{g}$ is induced from a character $\psi_{g}$ of signature $(+,-)$ of a real quadratic field $K$, then the character $\psi=\psi_{g} / \psi_{g}^{\prime}$ is a totally odd ring class character of $K$, and it cuts out an abelian extension of $K$ of dihedral type. The decomposition (21) continues to hold, but now, since $\sigma_{\infty}$ acts with eigenvalue 1 on $L\left(\chi_{K}\right)$, it follows that

$$
\left(\mathcal{O}_{H_{g}}^{\times}\right)_{L}^{\mathrm{Ad}_{g}} \simeq L\left(\chi_{K}\right)
$$

in all cases. If $p$ splits in $K$, then $\chi_{K}(p)=1 \neq \alpha_{g} / \beta_{g}$, and hence $U_{g_{\alpha}}=0$. If $p$ is inert in $K$, then $\chi_{K}(p)=\alpha_{g} / \beta_{g}=-1$, and hence $U_{g_{\alpha}}$ is also one dimensional, and is generated over $L$ by a fundamental unit of the real quadratic field $K$.

(c) Exotic forms. Assume to conclude that the projective image $G$ of the representation $V_{g}$ is isomorphic to $A_{4}, S_{4}$, or $A_{5}$. Since these groups admit no faithful irreducible representations of dimension $<3$, it follows that $\operatorname{Ad}_{g}$ is irreducible, and hence

$$
\left(\mathcal{O}_{H_{g}}^{\times}\right)_{L}^{\operatorname{Ad}_{g}} \simeq \operatorname{Ad}_{g} .
$$

The lemma follows directly in this case, since $\sigma_{p}$ acts on $\operatorname{Ad}_{g}$ with eigenvalues $1, \alpha_{g} / \beta_{g}$, and $\beta_{g} / \alpha_{g}$.

REMARK 1.7. Note that Condition (14), which was shown in Section 1.1 to imply Hypothesis $\mathrm{C}$ for $g_{\alpha}$, is precisely what guarantees the nontriviality of the group $U_{g_{\alpha}}$.

REMARK 1.8. The scenarios considered in (a) and (b) in the above proof are not mutually exclusive, and can arise simultaneously in certain cases where $\psi_{g}$ 
is quadratic and $\varrho_{g}$ has image isomorphic to the dihedral group of order eight: some of the numerical examples below focus on such settings.

DEFINITION 1.9. A nonzero element $u_{g_{\alpha}}$ of $U_{g_{\alpha}}$ is called a Stark unit attached to $g_{\alpha}$.

REMARK 1.10. Lemma 1.6 furnishes us with many concrete instances where the unit $u_{g_{\alpha}}$ is well defined up to multiplication by $L^{\times}$. This happens, for instance, when $\varrho_{g}$ is induced from a character of a real quadratic field $K$ in which $p$ is inert, when the vector space $U_{g_{\alpha}}$ is always generated by the fundamental unit of $K$. The case where $p$ splits in $K$ is more mysterious, since $U_{g_{\alpha}}=0$, and a natural definition of $u_{g_{\alpha}}$ does not seem to be available, consistent with the failure of Hypothesis C.

More generally, $\operatorname{dim}_{L}\left(U_{g_{\alpha}}\right)>1$ occurs precisely when $\alpha_{g} / \beta_{g}= \pm 1$. In the special case where $V_{g}$ is induced from a character of an imaginary quadratic field in which $p$ splits, there is nonetheless a natural candidate for $u_{g_{\alpha}}$ which is well defined up to scaling by $L^{\times}$: see Section 3.2 for more details.

REMARK 1.11. Several results in the literature relate the Petersson norm of $g$ to $L^{\prime}\left(\operatorname{Ad}_{g}, 0\right)$, which in turn is expected to admit an elementary expression as the logarithm of units in the subspace of $\left(\mathcal{O}_{H_{g}}^{\times}\right)_{L}^{\operatorname{Ad}_{g}}$ on which $\sigma_{\infty}=1$. See for example [St1, Section 6], or [DuLi, Proposition 4.1]. It is in this sense that the $p$-adic logarithm of $u_{g_{\alpha}}$ might be viewed as a $p$-adic avatar of $\left\langle g_{\alpha}, g_{\alpha}\right\rangle$.

1.2.2. The case where $\varrho_{g}$ is reducible. Consider now the case where $g$ corresponds to the Eisenstein series $E_{1}\left(\chi_{1}, \chi_{2}\right) \in M_{1}(N, \chi)$, where $\chi_{1}$ and $\chi_{2}$ are odd and even Dirichlet characters satisfying (as motivated in Section 1.1) the condition

$$
\chi_{1}(p)=\chi_{2}(p)
$$

In that case,

$$
V_{g}=L\left(\chi_{1}\right) \oplus L\left(\chi_{2}\right), \quad \operatorname{Ad}_{g}=L \oplus L(\eta) \oplus L\left(\eta^{-1}\right) \quad \text { where } \eta:=\chi_{1} / \chi_{2} .
$$

Condition (23) implies that $\eta(p)=1$, and hence $p$ splits completely in the abelian extension $H_{\eta} / \mathbb{Q}$ cut out by $\eta$.

To define $u_{g_{\alpha}}$, choose a prime ideal $\wp$ of $\mathcal{O}_{H_{\eta}}$ over $p$ inducing the embedding $H_{\eta} \hookrightarrow \mathbb{C}_{p}$ chosen at the outset, and let $u_{\wp} \in \mathcal{O}_{H_{\eta}}[1 / p]^{\times}$be a generator of the principal ideal $\wp^{h}$, where $h$ is the class number of $H_{\eta}$ :

$$
\wp^{h}=\left(u_{\wp}\right), \quad u_{\wp} \in\left(\mathcal{O}_{H_{\eta}}[1 / \wp]\right)^{\times}\left(\bmod \mathcal{O}_{H_{\eta}}^{\times}\right) .
$$

The unit $u_{g_{\alpha}}$ is defined as follows (in additive notation). 
DEFINITION 1.12. Let

$$
e_{\eta}:=\frac{1}{\# G} \sum_{\sigma \in G} \eta^{-1}(\sigma) \sigma, \quad e_{\eta^{-1}}:=\frac{1}{\# G} \sum_{\sigma \in G} \eta(\sigma) \sigma,
$$

be the usual idempotents in $L[G]$ giving the projection onto the $\eta$ and $\eta^{-1}$ isotypic component for the action of $G$. Set

$$
u_{g_{\alpha}}=e_{\eta} u_{\wp}+e_{\eta^{-1}} u_{\wp} .
$$

The unit $u_{g_{\alpha}}$ is a linear combination of Gross-Stark units attached to the characters $\eta$ and $\eta^{-1}$, whose $p$-adic logarithms

$$
\log _{\wp}\left(u_{g_{\alpha}}\right)=\frac{1}{\# G} \sum_{\sigma \in \operatorname{Gal}\left(H_{\eta} / \mathbb{Q}\right)}\left(\eta(\sigma)+\eta^{-1}(\sigma)\right) \log _{\wp}\left(\sigma\left(u_{\wp}\right)\right)
$$

account for the derivative at zero of the Kubota-Leopoldt $p$-adic $L$-function of $\eta$. In this formula, $\log _{\wp}$ denotes Iwasawa's logarithm satisfying $\log _{\wp}(p)=0$, composed with the $p$-adic embedding of $H_{\eta}$ attached to $\wp$. It is not hard to see that the following hold.

(1) Although the unit $u_{\wp}$ is only well defined modulo $\mathcal{O}_{H_{\eta}}^{\times}$, there is no resulting ambiguity in the unit $u_{g_{\alpha}}$, since $\eta$ and $\eta^{-1}$ are odd characters, and hence the idempotents $e_{\eta}$ and $e_{\eta^{-1}}$ annihilate $\mathcal{O}_{H_{\eta}}^{\times} \otimes L$.

(2) The unit $u_{\wp}$, and hence also the unit $u_{g_{\alpha}}$, depends crucially (and not just by scaling by $L^{\times}$!) on the choice of the ideal $\wp$ above $p$, but the expression $\log _{\wp} u_{g_{\alpha}}$ is independent of this choice, up to multiplication by a root of unity.

\section{Iterated integrals and the Garrett-Hida $p$-adic $L$-function}

The main goal of this section is to recast the $p$-adic iterated integral that arose in the introduction as the value of a $p$-adic $L$-function at a point which lies outside its region of classical interpolation. This interpretation is crucial for the proof of Theorem 3.3 of Section 3.2, which provides the main piece of theoretical evidence for Conjecture ES in a setting where the $p$-adic trilinear form $I_{p}^{\prime}$ does not vanish.

We begin by briefly recalling a few key facts about Garrett's triple-product classical $L$-function and the work of Harris-Kudla, Ichino, Watson, and Woodbury on Jacquet's conjecture on trilinear periods.

Let $(k, \ell, m)$ be a triple of positive integers, and let

$$
g_{k} \in M_{k}\left(N_{g}, \chi_{g}\right), \quad f_{\ell} \in M_{\ell}\left(N_{f}, \chi_{f}\right), \quad h_{m} \in M_{m}\left(N_{h}, \chi_{h}\right)
$$


be three newforms (assumed to be cuspidal if the weight is at least two) satisfying $\chi_{g} \chi_{f} \chi_{h}=1$. Note that this forces $k+\ell+m$ to be even. Set $N=\operatorname{lcm}\left(N_{f}, N_{g}, N_{h}\right)$, and write $\mathbb{Q}\left(g_{k}, f_{\ell}, h_{m}\right)$ for the number field generated by the Fourier coefficients of the three forms.

Let $L\left(g_{k} \otimes f_{\ell} \otimes h_{m}, s\right)$ denote Garrett's triple-product $L$-function associated to $\left(g_{k}, f_{\ell}, h_{m}\right)$. It is a self-dual $L$-function defined by an Euler product of degree eight and satisfying a functional equation with central critical point $c=(k+\ell+m-2) / 2$ and root number

$$
\varepsilon=\prod_{v \mid N \infty} \varepsilon_{v}\left(g_{k} \otimes f_{\ell} \otimes h_{m}\right) \quad \text { with } \varepsilon_{v}\left(g_{k} \otimes f_{\ell} \otimes h_{m}\right) \in\{ \pm 1\} .
$$

The Archimedean sign $\varepsilon_{\infty}$ is equal to one if and only if the triple $(k, \ell, m)$ of weights is unbalanced, that is, one of the weights is greater or equal to the sum of the other two. If $v \mid N$ is a finite place, the main result of [Pr] asserts that $\varepsilon_{v}\left(g_{k} \otimes f_{\ell} \otimes h_{m}\right)=+1$ if and only if there exists a $\mathbf{G L}_{2}\left(\mathbb{Q}_{v}\right)$-invariant linear form in the tensor product $\pi_{f, v} \otimes \pi_{g, v} \otimes \pi_{h, v}$ of local admissible representations of $\mathbf{G L}_{2}\left(\mathbb{Q}_{v}\right)$ associated to the three eigenforms. This is the case if $v \nmid \operatorname{gcd}\left(N_{f}\right.$, $N_{g}, N_{h}$ ), or if one of $\pi_{f, v}, \pi_{g, v}$ or $\pi_{h, v}$ does not belong to the discrete series.

Motivated by the $\mathrm{PhD}$ thesis of Prasad, Jacquet formulated a conjecture suggesting a necessary and sufficient criterion for the nonvanishing of the central critical value $L\left(g_{k} \otimes f_{\ell} \otimes h_{m}, c\right)$ in terms of certain trilinear periods. This conjecture was proved by Harris and Kudla in [HaKu], and several authors have subsequently refined this result by providing explicit formulae relating the $L$ value to the periods.

In order to describe the precise relationships between $L$-values and trilinear periods, we assume for the rest of this section that

$$
\varepsilon_{v}\left(g_{k} \otimes f_{\ell} \otimes h_{m}\right)=+1 \quad \text { for all } v \mid N \infty .
$$

When $(k, \ell, m)=(1,2,1)$ and $\left(g_{1}, f_{2}, h_{1}\right)=(g, f, h)$ are the modular forms considered in the introduction, then $L\left(g_{k} \otimes f_{\ell} \otimes h_{m}, s\right)=L\left(E / \mathbb{Q}, \varrho_{g h}, s\right)$ is the Hasse-Weil-Artin $L$-function considered there, and (24) is just Hypothesis A. The above discussion yields sufficient conditions for Hypothesis A to hold, and shows that this is not a very stringent assumption, in practice. Condition (24) at $v=\infty$ implies that the triple $(k, \ell, m)$ is unbalanced; assume for the sake of definiteness that $k \geqslant \ell+m$ from now on.

As in the introduction, let

$$
S_{k}\left(N, \chi_{g}\right)\left[g_{k}\right], \quad S_{\ell}\left(N, \chi_{f}\right)\left[f_{\ell}\right], \quad S_{m}\left(N, \chi_{h}\right)\left[h_{m}\right]
$$

denote the eigenspaces corresponding to the three forms in level $N$ with respect to the Hecke operators $\left\{T_{n}, n \nmid N\right\}$. 
For any $w \geqslant 1$ and any Dirichlet character $\chi$ of conductor $N$, let $S_{w}^{\mathrm{nh}}(N, \chi)$ denote the space of nearly holomorphic modular forms of weight $w$, level $N$, and nebentype $\chi$, and let

$$
\delta=\frac{1}{2 \pi i}\left(\frac{d}{d z}+\frac{w}{z-\bar{z}}\right): S_{w}^{\mathrm{nh}}(N, \chi) \longrightarrow S_{w+2}^{\mathrm{nh}}(N, \chi)
$$

denote the Shimura-Maass operator, as described for instance in [Hi2, Chapter $\mathrm{X}]$. Let also

$$
\langle,\rangle: S_{w}^{\mathrm{nh}}(N, \chi) \times S_{w}^{\mathrm{nh}}(N, \chi) \longrightarrow \mathbb{C}, \quad\left\langle f_{1}, f_{2}\right\rangle=\int_{\Gamma_{1}(N) \backslash \mathfrak{H}} \overline{f_{1}(z)} f_{2}(z) y^{w} \frac{d x d y}{y^{2}},
$$

denote the Petersson scalar product on these spaces.

Set $t=(k-\ell-m) / 2 \geqslant 0$. A test vector for the triple $\left(g_{k}, f_{\ell}, h_{m}\right)$ is a triplet

$$
\left(\breve{g}_{k}, \breve{f}_{\ell}, \breve{h}_{m}\right) \in S_{k}\left(N, \chi_{g}\right)_{L}\left[g_{k}\right] \times S_{\ell}\left(N, \chi_{f}\right)_{L}\left[f_{\ell}\right] \times S_{m}\left(N, \chi_{h}\right)_{L}\left[h_{m}\right] .
$$

For such a test vector, recall that we denote $g_{k}^{*}=g_{k} \otimes \chi_{g}^{-1}$, and define the quantities

$$
I\left(\breve{g}_{k}, \breve{f}_{\ell}, \breve{h}_{m}\right):=\left\langle\breve{g}_{k}^{*}, \delta^{t}\left(\breve{f}_{\ell}\right) \cdot \breve{h}_{m}\right\rangle,
$$

as well as

$$
\begin{aligned}
J\left(\breve{g}_{k}, \breve{f}_{\ell}, \breve{h}_{m}\right) & :=I\left(\breve{g}_{k}, \breve{f}_{\ell}, \breve{h}_{m}\right) /\left\langle g_{k}^{*}, g_{k}^{*}\right\rangle, \\
\omega\left(\breve{g}_{k}, \breve{f}_{\ell}, \breve{h}_{m}\right) & :=I\left(\breve{g}_{k}^{*}, \breve{f}_{\ell}^{*}, \breve{h}_{m}^{*}\right) / I\left(\breve{g}_{k}, \breve{f}_{\ell}, \breve{h}_{m}\right) .
\end{aligned}
$$

The following result is a direct consequence of the fundamental work of Ichino [Ich]. The precise formulation given here is only valid under Assumption (24) and follows by combining [Ich]. with a formula due to Hida for a special value of the $L$-function of the adjoint of a modular form and the precise formulae of Watson [Wa] and Woodbury [Wo1, Wo2].

PROPOSITION 2.1.

(a) For any test vector $\left(\breve{g}_{k}, \breve{f}_{\ell}, \breve{h}_{m}\right)$,

$$
\left|I\left(\breve{g}_{k}, \breve{f}_{\ell}, \breve{h}_{m}\right)\right|^{2}=\mathfrak{a}(k, \ell, m) \cdot \mathfrak{f}(k, \ell, m) \cdot \prod_{v \mid N \infty} C_{v}\left(\breve{g}_{k}, \breve{f}_{\ell}, \breve{h}_{m}\right) \cdot L\left(g_{k} \otimes f_{\ell} \otimes h_{m}, c\right),
$$

where

(i) $\mathfrak{a}(k, \ell, m)=((k+\ell+m-4) / 2) ! \cdot((k+\ell-m-2) / 2) ! \cdot((k-\ell+$ $m-2) / 2) ! \cdot((k-\ell-m) / 2) ! \cdot \pi^{-2 k}$; 
(ii) $\mathfrak{f}(k, \ell, m)=A \cdot 2^{B}$, where $A \in \mathbb{Q}^{\times}$depends only on the levels of the eigenforms and of their characters, and $B=B(k, \ell, m) \in \mathbb{Z}$ is a linear function on the weights $k, \ell, m$;

(iii) for all $v \mid N \infty$, the local term $C_{v}\left(\breve{g}_{k}, \breve{f}_{\ell}, \breve{h}_{m}\right) \in L$ depends only on the admissible representations of $\mathbf{G L}_{2}\left(\mathbb{Q}_{v}\right)$ associated to the three cusp forms and on the local components at $v$ of the choice of $\left(\breve{g}_{k}, \breve{f}_{\ell}, \breve{h}_{m}\right)$.

(b) There exists a test vector $\left(\breve{g}_{k}, \breve{f}_{\ell}, \breve{h}_{m}\right)$ for which $C_{v}\left(\breve{g}_{k}, \breve{f}_{\ell}, \breve{h}_{m}\right) \neq 0$ for all $v \mid N \infty$.

(c) Assume that $N$ is square free. Then there exists a particular test vector $\left(\breve{g}_{k}, \breve{f}_{\ell}, \breve{h}_{m}\right)$ such that $W_{v}:=C_{v}\left(\breve{g}_{k}, \breve{f}_{\ell}, \breve{h}_{m}\right) \in \mathbb{Q}^{\times}$is a rational constant for all $v \mid N$.

Proof. Let $\Gamma_{\mathbb{R}}(s):=\pi^{-s / 2} \Gamma(s / 2)$ and $\Gamma_{\mathbb{C}}(s):=2(2 \pi)^{-s} \Gamma(s)$, where $\Gamma(s)$ is the usual gamma function. For any eigenform $\varphi \in S_{w}(N, \chi)$, let

$$
L(\operatorname{Ad}(\varphi), s)=L\left(\operatorname{sym}^{2}(\varphi), \chi^{-1}, s\right)
$$

denote the $L$-function of the adjoint square of $\varphi$, and let

$$
L^{*}(\operatorname{Ad}(\varphi), s)=L_{\infty}(\operatorname{Ad}(\varphi), s) L(\operatorname{Ad}(\varphi), s)
$$

denote the completed $L$-function, with

$$
L_{\infty}(\operatorname{Ad}(\varphi), s):=\Gamma_{\mathbb{R}}(s-w+2) \Gamma_{\mathbb{C}}(s)
$$

the idoneous factor at $\infty$.

The Rankin-Selberg method relates the special value of $L(\operatorname{Ad}(\varphi), s)$ at $s=w$ to the Petersson norm of $\varphi$. A completely explicit formula is given in [Hi1, Theorem 5.1], which, combined with the value of $L_{\infty}(\operatorname{Ad}(\varphi), s)$ at $s=w$, yields

$$
L^{*}(\operatorname{Ad}(\varphi), w)=2^{w} A_{\varphi} \times\langle\varphi, \varphi\rangle,
$$

where $A_{\varphi} \in \mathbb{Q}^{\times}$is a constant which depends only on the level of $\varphi$ and conductor of $\chi$.

Likewise, let

$$
L^{*}\left(g_{k} \otimes f_{\ell} \otimes h_{m}, s\right):=L_{\infty}\left(g_{k} \otimes f_{\ell} \otimes h_{m}, s\right) L\left(g_{k} \otimes f_{\ell} \otimes h_{m}, s\right)
$$

denote the completed Garrett $L$-function, where

$$
L_{\infty}\left(g_{k} \otimes f_{\ell} \otimes h_{m}, s\right):=\Gamma_{\mathbb{C}}(s) \Gamma_{\mathbb{C}}(s+1-m) \Gamma_{\mathbb{C}}(s+1-\ell) \Gamma_{\mathbb{C}}(s+2-\ell-m) .
$$


The main result of [Ich] (see also [Wo1, Theorem 2.1] for a reformulation of the same statement) asserts that, for any test vector $\left(\breve{g}_{k}, \breve{f}_{\ell}, \breve{h}_{m}\right)$,

$$
\frac{\left|I\left(\breve{g}_{k}, \breve{f}_{\ell}, \breve{h}_{m}\right)\right|^{2}}{\left\langle g_{k}, g_{k}\right\rangle \cdot\left\langle f_{\ell}, f_{\ell}\right\rangle \cdot\left\langle h_{m}, h_{m}\right\rangle}=C \cdot \frac{\prod_{v \mid N \infty} C_{v}\left(\breve{g}_{k}, \breve{f}_{\ell}, \breve{h}_{m}\right) \cdot L^{*}\left(g_{k} \otimes f_{\ell} \otimes h_{m}, c\right)}{L^{*}\left(\operatorname{Ad}\left(g_{k}\right), k\right) L^{*}\left(\operatorname{Ad}\left(f_{\ell}\right), \ell\right) L^{*}\left(\operatorname{Ad}\left(h_{m}\right), m\right)},
$$

where

- $C \in \mathbb{Q}^{\times}$is an absolute constant which only depends on the normalization taken for the Petersson scalar product; and

- for any $v \mid N \infty, C_{v}\left(\breve{g}_{k}, \breve{f}_{\ell}, \breve{h}_{m}\right) \in \mathbb{Q}\left(g_{k}, f_{\ell}, h_{m}\right)$ is as described in (iii) above.

Combining (29) with (27), and evaluating (28) at $s=c$, it follows that

$$
\left|I\left(\breve{g}_{k}, \breve{f}_{\ell}, \breve{h}_{m}\right)\right|^{2}=\mathfrak{a}(k, \ell, m) \cdot \mathfrak{f}(k, \ell, m) \cdot \prod_{v \mid N \infty} C_{v} \cdot L\left(g_{k} \otimes f_{\ell} \otimes h_{m}, c\right),
$$

where we have set $\mathfrak{f}(k, \ell, m)=2^{-3 k-\ell-m+4} \cdot C \cdot\left(A_{g_{k}} A_{f_{\ell}} A_{h_{m}}\right)^{-1}$. This proves (a). Statement (b) was originally known as Jacquet's conjecture, and was proved by Harris and Kudla in [HaKu]. As for (c), we refer to [Wo1, Theorem 5.1].

REMARK 2.2. The choice of test vector in (c) is a completely explicit one, given by Watson [Wa] in the case when $N_{g}=N_{f}=N_{h}$, and more recently by Woodbury [Wo1] for arbitrary square-free levels. We shall refer to it as the Watson-Woodbury test vector. The resulting value of $W_{v} \in \mathbb{Q}^{\times}$for $v \mid N$ is also explicit (see [Wa, Theorem 3] and [Wo1, Theorem 5.1] for the recipes), and we shall refer to it as the Watson-Woodbury local constant at $v$. Note that we say nothing about $C_{\infty}\left(\breve{g}_{k}, \breve{f}_{\ell}, \breve{h}_{m}\right)$, although having a tight control of it would be desirable. See [Wo2] for the latest developments, which assert that in favourable scenarios (such as the one considered in Section 3.2 below) the Watson-Woodbury test vector yields $W_{\infty}=1$.

Up to this point we have summarized the basic material we need about Garrett triple-product classical $L$-functions. Fix as in the introduction a prime number $p \nmid N$, and let us discuss now the $p$-adic interpolation properties of their central critical values. The vehicle that allows to do so is the notion of Hida families, which are families of $p$-adic overconvergent ordinary modular forms parameterized by a rigid analytic space: we refer to [DR1, Section 2.6] and [DR2, Section 1.8] for a brief introduction to this subject, presented in a language suitable for our purposes. Let

$$
\Omega:=\operatorname{Hom}\left(\mathbb{Z}_{p}^{\times}, \mathbb{Z}_{p}^{\times}\right) \simeq \mathbb{Z} /(p-1) \mathbb{Z} \times \mathbb{Z}_{p}
$$


denote the weight space, which contains the integers as a dense subset by means of the identification $k \leftrightarrow \varphi_{k}$, where $\varphi_{k} \in \Omega$ is the homomorphism defined by the rule $\varphi_{k}(n)=n^{k-1}$.

Let $\mathbf{g}, \mathbf{f}$, and $\mathbf{h}$ be three Hida families of new tame level $N_{g}, N_{f}$, and $N_{h}$ and tame nebentype characters $\chi_{g}, \chi_{f}$, and $\chi_{h}$, respectively. Assume that $\chi_{g} \chi_{f} \chi_{h}$ $=1$, as above.

Let $\mathcal{U}_{g}$ denote the parameter space of the Hida family $\mathbf{g}$, and let $k_{\mathbf{g}}: \mathcal{U}_{g} \rightarrow \Omega$ denote the finite-weight map associated to it. If confusion is not likely to arise, in order to simplify the notation we may freely identify a point $x \in \mathcal{U}_{\mathrm{g}}$ with its image $k=k_{\mathbf{g}}(x) \in \Omega$.

For any point $x \in \mathcal{U}_{g}$, let $x(\mathbf{g})$ denote the specialization of $\mathbf{g}$ at $x$. In general, $x(\mathbf{g})$ is an overconvergent ordinary modular form. Define $\mathcal{U}_{g}^{\text {cl }} \subseteq \mathcal{U}_{g}$ to be the subset of points $x \in \mathcal{U}_{g}$ such that $x(\mathbf{g})$ is a classical ordinary eigenform. By the work of Hida, $\mathcal{U}_{g}^{\text {cl }}$ contains all points of weight $k_{\mathbf{g}}(x)=k \in \mathbb{Z}_{\geqslant 2}$. Points of weight one may belong to $\mathcal{U}_{g}^{\text {cl }}$ or not. Define further $\mathcal{U}_{g}^{\circ}$ to be the subset of classical points $x \in \mathcal{U}_{g}^{\mathrm{cl}}$ such that the eigenform $x(\mathbf{g})$ is old at $p$. For such points, there exists a classical newform that we denote $g_{k} \in M_{k}\left(N_{g}, \chi_{g}\right)$ whose ordinary $p$-stabilization is the specialization of $\mathbf{g}$ at $x$. Phrased in terms of $q$-expansions, this amounts to saying that

$$
x(\mathbf{g})(q)=g_{k}(q)-\beta g_{k}\left(q^{p}\right) \in M_{k}\left(N_{g} p, \chi_{g}\right),
$$

where $\beta \in \mathbb{C}_{p}$ is a root of the $p$ th Hecke polynomial $x^{2}-a_{p}\left(g_{k}\right) x+\chi_{g}(p) p^{k-1}$ whose $p$-adic valuation is $k-1$. Adopt throughout similar notation as above for $\mathbf{f}$ and $\mathbf{h}$.

Note that, for any triple of classical points $(x, y, z) \in \mathcal{U}_{g}^{\circ} \times \mathcal{U}_{f}^{\circ} \times \mathcal{U}_{h}^{\circ}$ of weights $(k, \ell, m)$, the subfield $\mathbb{Q}\left(g_{k}, f_{\ell}, h_{m}\right)$ of $\mathbb{C}_{p}$ generated by the Fourier coefficients of $g_{k}, f_{\ell}$, and $h_{m}$ is a finite extension of $\mathbb{Q}$, whose degree varies in general as a function of the weights $(k, \ell, m)$.

Let $\breve{\mathbf{g}}, \breve{\mathbf{f}}$, and $\breve{\mathbf{h}}$ be $\Lambda$-adic test vectors of tame level $N$ associated to $\mathbf{g}, \mathbf{f}$, and $\mathbf{h}$, as described in [DR1, Definition 2.16]. Such test vectors may be written down explicitly as $\breve{\mathbf{g}}=\sum_{d \mid N / N_{g}} \lambda_{d} \mathbf{g}\left(q^{d}\right)$, where $d$ ranges over the positive divisors of $N / N_{g}$, and $\lambda_{d} \in \Lambda_{g}=\mathcal{O}\left(\mathcal{U}_{g}\right)$ are Iwasawa functions on the domain $\mathcal{U}_{g}$ (and similarly for $\breve{\mathbf{f}}$ and $\breve{\mathbf{h}}$ ).

Assume that $\operatorname{gcd}\left(N_{f}, N_{g}, N_{h}\right)=1$, and that the three Hida families are cuspidal, meaning that all their specializations at classical points of weight greater than one (though not necessarily at points of weight one) are cuspidal eigenforms. The first assumption implies that the local signs hypothesis (24) holds for all triples $\left(g_{k}, f_{\ell}, h_{m}\right)$ at points $(k, \ell, m) \in \mathcal{U}_{g}^{\circ} \times \mathcal{U}_{f}^{\circ} \times \mathcal{U}_{h}^{\circ}$.

Define a dense subset $\mathcal{U}_{g f h}^{\circ} \subset \mathcal{U}_{g} \times \mathcal{U}_{f} \times \mathcal{U}_{h}$ by

$$
\mathcal{U}_{g f h}^{\circ}=\left\{(k, \ell, m) \in \mathcal{U}_{g}^{\circ} \times \mathcal{U}_{f}^{\circ} \times \mathcal{U}_{h}^{\circ} \text { such that } k \geqslant \ell+m\right\} .
$$


Under the running assumptions, a three-variable $p$-adic $L$-function

$$
\mathscr{L}_{p}^{g}(\breve{\mathbf{g}}, \breve{\mathbf{f}}, \breve{\mathbf{h}}): \mathcal{U}_{g} \times \mathcal{U}_{f} \times \mathcal{U}_{h} \longrightarrow \mathbb{C}_{p}
$$

was constructed in [DR1, Section 4.2], which is characterized by the following interpolation property at triplets $(k, \ell, m) \in \mathcal{U}_{f g h}^{\circ}$ :

$$
\mathscr{L}_{p}^{g}(\breve{\mathbf{g}}, \breve{\mathbf{f}}, \breve{\mathbf{h}})(k, \ell, m)=\mathfrak{e}(k, \ell, m) \cdot J\left(\breve{g}_{k}, \breve{f}_{\ell}, \breve{h}_{m}\right)
$$

where

$$
\mathfrak{e}(k, \ell, m)=\mathcal{E}\left(g_{k}, f_{\ell}, h_{m}\right) / \mathcal{E}_{0}\left(g_{k}\right) \mathcal{E}_{1}\left(g_{k}\right)=\mathcal{E}\left(g_{k}^{*}, f_{\ell}^{*}, h_{m}^{*}\right) / \mathcal{E}_{0}\left(g_{k}^{*}\right) \mathcal{E}_{1}\left(g_{k}^{*}\right)
$$

denotes the Euler factor introduced in [DR1, Theorem 1.3].

The next proposition spells out the interpolation property tying this $p$-adic $L$-function to central critical $L$-values.

Proposition 2.3. For any $\Lambda$-adic test vectors $\breve{\mathbf{g}}, \breve{\mathbf{f}}, \breve{\mathbf{h}}$, and every $(k, \ell, m) \in$ $\mathcal{U}_{f g h}^{\circ}$, the following formula is valid:

$$
\begin{aligned}
& \mathscr{L}_{p}^{g}(\breve{\mathbf{g}}, \breve{\mathbf{f}}, \breve{\mathbf{h}}) \cdot \mathscr{L}_{p}^{g}\left(\breve{\mathbf{g}}^{*}, \breve{\mathbf{f}}^{*}, \breve{\mathbf{h}}^{*}\right)(k, \ell, m) \\
&= \mathfrak{e}(k, \ell, m)^{2} \cdot \mathfrak{a}(k, \ell, m) \cdot \mathfrak{f}(k, \ell, m) \cdot \prod_{v \mid N \infty} C_{v}\left(\breve{g}_{k}, \breve{f}_{\ell}, \breve{h}_{m}\right) \\
& \quad \times \frac{L\left(g_{k} \otimes f_{\ell} \otimes h_{m}, \frac{k+\ell+m-2}{2}\right)}{\left\langle g_{k}^{*}, g_{k}^{*}\right\rangle^{2}} .
\end{aligned}
$$

Proof. Note that

$$
\left|I\left(\breve{g}_{k}, \breve{f}_{\ell}, \breve{h}_{m}\right)\right|^{2}=I\left(\breve{g}_{k}, \breve{f}_{\ell}, \breve{h}_{m}\right) \cdot \overline{I\left(\breve{g}_{k}, \breve{f}_{\ell}, \breve{h}_{m}\right)}=I\left(\breve{g}_{k}, \breve{f}_{\ell}, \breve{h}_{m}\right) \cdot I\left(\breve{g}_{k}^{*}, \breve{f}_{\ell}^{*}, \breve{h}_{m}^{*}\right)
$$

and that

$$
\left\langle g_{k}, g_{k}\right\rangle=\left\langle g_{k}^{*}, g_{k}^{*}\right\rangle .
$$

It thus follows from (32) and (26) that

$$
\begin{aligned}
\mathscr{L}_{p}^{g}(\breve{\mathbf{g}}, \breve{\mathbf{f}}, \breve{\mathbf{h}}) \cdot \mathscr{L}_{p}^{g}\left(\breve{\mathbf{g}}^{*}, \breve{\mathbf{f}}^{*}, \breve{\mathbf{h}}^{*}\right)(k, \ell, m) \\
=\mathfrak{e}(k, \ell, m)^{2} \frac{\left|I\left(\breve{g}_{k}, \breve{f}_{\ell}, \breve{h}_{m}\right)\right|^{2}}{\left\langle g_{k}^{*}, g_{k}^{*}\right\rangle^{2}} \\
=\mathfrak{e}(k, \ell, m)^{2} \cdot \mathfrak{a}(k, \ell, m) \cdot \mathfrak{f}(k, \ell, m) \cdot \prod_{v \mid N \infty} C_{v}\left(\breve{g}_{k}, \breve{f}_{\ell}, \breve{h}_{m}\right) \\
\quad \times \frac{L\left(g_{k} \otimes f_{\ell} \otimes h_{m}, \frac{k+\ell+m-2}{2}\right)}{\left\langle g_{k}^{*}, g_{k}^{*}\right\rangle^{2}} .
\end{aligned}
$$


REMARK 2.4. An inspection of the definition of the Watson-Woodbury test vector shows that, when we let it vary along the Hida families $\mathbf{g}, \mathbf{f}$, and $\mathbf{h}$, it gives rise to a triplet $(\breve{\mathbf{g}}, \breve{\mathbf{f}}, \breve{\mathbf{h}})$ of $\Lambda$-adic test vectors of tame level $N$, whose specialization at any $(k, \ell, m) \in \mathcal{U}_{g}^{\circ} \times \mathcal{U}_{f}^{\circ} \times \mathcal{U}_{h}^{\circ}$ is an eigenform with coefficients in $\mathbb{Q}\left(g_{k}, f_{\ell}, h_{m}\right)$, a finite extension of $\mathbb{Q}$; and, moreover, the constants $W_{v}=$ $C_{v}\left(\breve{g}_{k}, \breve{f}_{\ell}, \breve{h}_{m}\right) \in \mathbb{Q}^{\times}$in Proposition 2.1(c) depend on $v$ and $N$ but not on the triplet $(k, l, m)$ of weights.

For the remainder of this section (and throughout the rest of the paper, as we shall recall again in later sections), we impose the following hypothesis.

ASSUMPTION 2.5. There exists a point $x \in \mathcal{U}_{\mathrm{g}}^{\circ}$ of weight $k_{\mathrm{g}}(x)=1$ such that the specialization $g_{\alpha}:=x(\mathbf{g}) \in M_{1}\left(N_{g} p, \chi_{g}\right)$ satisfies Hypothesis $\mathrm{C}$.

To make sure that the notation introduced in the Introduction and that in this section are compatible, let us emphasize that $g_{\alpha}$ is an eigenform of weight one and level $N_{g} p$ that is new at $N_{g}$ and old at $p$. The eigenform $g_{\alpha}$ is the $p$ stabilization of a newform $g:=g_{1} \in M_{1}\left(N_{g}, \chi_{g}\right)$.

The next proposition relates this restriction of the Garrett-Hida $p$-adic $L$ function to $p$-adic iterated integrals.

PROPOSITION 2.6. There exists a linear form

$$
\breve{\gamma}_{\alpha}: S_{1}\left(N p, \chi_{g}^{-1}\right)_{L}\left[g_{\alpha}^{*}\right] \longrightarrow L
$$

with $L=\mathbb{Q}\left(g_{1}, f_{2}, h_{1}\right)$ such that

$$
\mathscr{L}_{p}^{g}(\breve{\mathbf{g}}, \breve{\mathbf{f}}, \breve{\mathbf{h}})(1,2,1)=\int_{\breve{\gamma}_{\alpha}} \breve{f}_{2} \cdot \breve{h}_{1} .
$$

Proof. Fix a finite flat extension $\Lambda^{\dagger}$ of $\Lambda$, sufficiently large so that it contains the coefficients of all the $\Lambda$-adic modular forms considered below, and write $\mathbf{S}^{\text {ord }}\left(N ; \Lambda^{\dagger}\right)$ for the space of $\Lambda$-adic modular forms with coefficients in $\Lambda^{\dagger}$. The Hida family $\mathbf{g}$ gives rise to a subspace

$$
\mathbf{S}^{\text {ord }}\left(N ; \Lambda^{\dagger}\right)[\mathbf{g}]:=\left\{\breve{\mathbf{g}} \in \mathbf{S}^{\text {ord }}\left(N ; \Lambda^{\dagger}\right) \text { such that } T_{n} \breve{\mathbf{g}}=a_{n}(\mathbf{g}) \breve{\mathbf{g}}, \forall(n, N)=1\right\} .
$$

Letting $\mathcal{L}^{\dagger}$ denote the fraction field of $\Lambda^{\dagger}$, the vector space $\mathbf{S}^{\text {ord }}\left(N ; \mathcal{L}^{\dagger}\right)[\mathbf{g}]$ is finite dimensional over $\mathcal{L}^{\dagger}$ and has for basis the set

$$
\left\{\mathbf{g}\left(q^{d}\right)\right\}_{d \mid\left(N / N_{g}\right)}
$$


of $\Lambda$-adic forms. By [DR1, Lemma 2.19], there exists a linear operator

$$
J\left(\breve{\mathbf{g}}^{*}\right): \mathbf{S}^{\text {ord }}\left(N, \Lambda^{\dagger}\right) \longrightarrow \mathcal{L}^{\dagger} \otimes_{\Lambda} \Lambda^{\dagger}, \quad \boldsymbol{\phi} \mapsto J\left(\breve{\mathbf{g}}^{*}, \boldsymbol{\phi}\right)
$$

that is characterized uniquely by the following interpolation property: at every point in $\mathcal{U}_{g}^{\circ} \cap \mathcal{U}_{\phi}^{\circ}$ of weight $k \geqslant 2$, the specialization of $J\left(\breve{\mathbf{g}}^{*}\right)$ is regular (meaning that there is no pole at this point) and is described by the following rule:

$$
v_{k}\left(J\left(\breve{\mathbf{g}}^{*}\right)\right): S_{k}^{\text {ord }}\left(N, \chi_{g}^{-1}\right)_{\mathbb{Q}_{p}\left(g_{k}\right)} \longrightarrow \mathbb{Q}_{p}\left(g_{k}\right), \quad \phi \mapsto \frac{\left\langle\breve{g}_{k}^{*}, \phi\right\rangle}{\left\langle g_{k}^{*}, g_{k}^{*}\right\rangle} .
$$

We turn now to analyse the specialization of $J\left(\breve{\mathbf{g}}^{*}\right)$ in weight one. For any $d \mid\left(N / N_{g}\right)$, let $c_{d}: \mathbf{S}^{\text {ord }}\left(N, \mathcal{L}^{\dagger}\right) \longrightarrow \mathcal{L}^{\dagger}$ denote the functional which associates to $\boldsymbol{\phi}$ its coefficient in $\mathbf{g}\left(q^{d}\right)$ with respect to the basis in (34). It follows from the proofs of [DR1, Lemmas 2.12 and 2.19] that

$$
J\left(\breve{\mathbf{g}}^{*}\right)=\sum_{d \mid\left(N / N_{g}\right)} \lambda_{d} \cdot c_{d}
$$

where $\lambda_{d} \in \mathcal{L}^{\dagger}$ are elements which, viewed as functions of $\mathbb{Z}_{\geqslant 2}$ by means of the rule $k \mapsto v_{k}\left(\lambda_{d}\right)$, can be expressed as polynomials in the expressions $q^{k}, a_{q}\left(g_{k}\right)$, $1 / q$, and $1 /(q+1)$ as $q$ ranges over the divisors of $N$. This implies that the specialization of $J\left(\breve{\mathbf{g}}^{*}\right)$ in weight one is also regular, and hence gives rise to a linear operator

$$
\breve{\gamma}_{\alpha}:=v_{1}\left(J\left(\breve{\mathbf{g}}^{*}\right)\right): S_{1}^{\mathrm{oc}, \text { ord }}\left(N, \chi_{g}^{-1}\right)_{L_{p}} \longrightarrow L_{p} .
$$

It factors through $S_{1}^{\text {oc,ord }}\left(N, \chi_{g}^{-1}\right)_{L_{p}}\left[\left[g_{\alpha}^{*}\right]\right]$, which by the classicality hypothesis imposed on $g_{\alpha}^{*}$ is isomorphic to the corresponding space $S_{1}\left(N p, \chi_{g}^{-1}\right)_{L_{p}}\left[g_{\alpha}^{*}\right]$ of classical modular forms.

As explained in the introduction, Hypothesis C equips $S_{1}^{\text {oc,ord }}\left(N, \chi_{g}^{-1}\right)_{L_{p}}\left[\left[g_{\alpha}^{*}\right]\right]$ with an $L$-rational structure, denoted $S_{1}^{\text {oc,ord }}\left(N, \chi_{g}^{-1}\right)_{L}\left[\left[g_{\alpha}^{*}\right]\right]$. In view of the description given above for $\lambda_{d}$ as functions of $k$, it follows that $v_{1}\left(\lambda_{d}\right) \in L$, and hence $\breve{\gamma}_{\alpha}$ is $L$-rational; that is to say, it belongs to $S_{1}(N p$, $\left.\chi_{g}^{-1}\right)_{L}\left[g_{\alpha}^{*}\right]^{\vee}=S_{1}^{\text {oc,ord }}\left(N, \chi_{g}^{-1}\right)_{L}\left[\left[g_{\alpha}^{*}\right]\right]^{\vee}$.

Let $e_{\text {ord }}\left(d^{\bullet} \breve{f}_{2}^{[p]} \times \breve{h}_{1}\right)$ denote the $\Lambda$-adic modular form whose specialization in weight $k$ is $e_{\text {ord }}\left(d^{(k-3) / 2} \breve{f}_{2}^{[p]} \times \breve{h}_{1}\right)$ for all $k \in \mathbb{Z}_{\geqslant 2}$. By [DR1, Definition 4.4], $\mathscr{L}_{p}^{g}\left(\breve{\mathbf{g}}, \breve{f}_{2}, \breve{h}_{1}\right)=J\left(\breve{\mathbf{g}}, e_{\text {ord }}\left(d^{\bullet} \breve{f}_{2}^{[p]} \times \breve{h}_{1}\right)\right)$ and, by construction,

$$
\mathscr{L}_{p}^{g}\left(\breve{\mathbf{g}}, \breve{f}_{2}, \breve{h}_{1}\right)(1)=\breve{\gamma}_{\alpha}\left(e_{\text {ord }}\left(d^{-1} \breve{f}_{2}^{[p]} \times \breve{h}_{1}\right)\right)=\int_{\breve{\gamma}_{\alpha}} \breve{f}_{2} \cdot \breve{h}_{1},
$$

as was to be shown. 
Proposition 2.6 recasts the $p$-adic iterated integral of Conjecture ES with $(\breve{\gamma}, \breve{f}, \breve{h})=\left(\breve{\gamma}_{\alpha}, \breve{f}_{2}, \breve{h}_{1}\right)$ as a $p$-adic avatar of the classical special value $L\left(E, \varrho_{g h}, 1\right)$. This suggests a possible strategy for proving Conjecture ES in the rank- $(1,1)$ setting where $\varrho_{g h}$ admits a decomposition of the form $\varrho_{g h}=\varrho_{1} \oplus \varrho_{2} \oplus \varrho_{3}$ as in (12). Assuming that the regulator $R_{g_{\alpha}}\left(E, \varrho_{g h}\right)$ is factorizable, Conjecture ES might then be proved by constructing appropriate ' $p$-adic Artin $L$-series' $\mathcal{L}_{p}\left(\operatorname{Ad}_{g_{\alpha}}\right)$ associated to (the irreducible constituent containing $\mathrm{Ad}_{g}^{+}$in) $\mathrm{Ad}_{g}$ and ' $p$-adic Hasse-Weil-Artin $L$-series' $\mathcal{L}_{p}\left(E, \varrho_{i}\right)$ attached to the irreducible constituents of $\varrho_{g h}$, and proving that

$$
\mathscr{L}_{p}^{g}\left(\breve{\mathbf{g}}, \breve{f}_{2}, \breve{h}_{1}\right) \cdot \mathcal{L}_{p}\left(\operatorname{Ad}_{g_{\alpha}}\right) \stackrel{?}{=} \mathcal{L}_{p}\left(E, \varrho_{1}\right) \cdot \mathcal{L}_{p}\left(E, \varrho_{2}\right) \cdot \mathcal{L}_{p}\left(E, \varrho_{3}\right)
$$

and

$$
\begin{array}{rr}
\mathcal{L}_{p}\left(\operatorname{Ad}_{g_{\alpha}}\right)(1) \stackrel{?}{=} \log _{p}\left(u_{g_{\alpha}}\right), \quad \mathcal{L}_{p}\left(E, \varrho_{1}\right)(1) \stackrel{?}{=} \log _{E, p}\left(P_{1}\right), \\
\mathcal{L}_{p}\left(E, \varrho_{3}\right)(1) \in L^{\times}, \quad \mathcal{L}_{p}\left(E, \varrho_{2}\right)(1) \stackrel{?}{=} \log _{E, p}\left(P_{2}\right) .
\end{array}
$$

This is the strategy carried out in Section 3.2: see Theorem 3.9 for the variant of (35) and (36) in the special setting considered therein. In general, identities like (35) appear to lie quite deep, and we are not even able to provide an independent definition of the $p$-adic $L$-values $\mathcal{L}_{p}\left(E, \varrho_{i}\right)$ that figure in its putative right-hand side; they are perhaps somewhat akin to the 'genuine' $p$-adic $L$-functions sought for in [Hi3].

\section{Heegner points and theta series of imaginary quadratic fields}

Let $K$ be an imaginary quadratic field of discriminant $-D_{K}$, and let $N_{K / \mathbb{Q}}$ denote the norm map on fractional ideals of $K$. The aim of this section is to study Conjecture ES in the case where both $g$ and $h$ are theta series associated to $K$. After setting up the basic notation and assumptions, the cases in which the prime $p$ splits or remains inert in $K$ are treated separately in Sections 3.2 and 3.3 .

Just as in the introduction, let $E$ be an elliptic curve over $\mathbb{Q}$, and let $f \in S_{2}\left(N_{f}\right)$ denote its associated newform. Given a finite-order character

$$
\psi: G_{K} \longrightarrow \mathbb{C}^{\times}
$$

of conductor $\mathfrak{c}_{\psi} \subseteq \mathcal{O}_{K}$ and central character $\chi$ (viewed as a Dirichlet character), let

$$
\theta_{\psi} \in M_{1}\left(D_{K} \cdot N_{K / \mathbb{Q}}\left(\mathfrak{c}_{\psi}\right), \chi\right)
$$


denote the theta series associated to $\psi$, and let $V_{\psi}:=\operatorname{Ind}_{K}^{\mathbb{Q}} \psi$ denote the twodimensional induced representation of $\psi$ from $G_{K}$ to $G_{\mathbb{Q}}$. It is known that $\theta_{\psi}$ is Eisenstein if and only if $V_{\psi}$ is reducible, which occurs precisely when $\psi=\psi^{\prime}$, where $\psi^{\prime}$ denotes the character of $G_{K}$ defined by the rule $\psi^{\prime}(\sigma)=\psi\left(\sigma_{0} \sigma \sigma_{0}^{-1}\right)$. Here, $\sigma_{0}$ is any choice of element in $G_{\mathbb{Q}} \backslash G_{K}$.

Fix two finite-order characters $\psi_{g}$ and $\psi_{h}$ of $K$ of conductors $\mathfrak{c}_{g}$ and $\mathfrak{c}_{h}$. Assume that the central character $\chi$ of $\psi_{h}$ is inverse to that of $\psi_{g}$, and set

$$
g:=\theta_{\psi_{g}} \in M_{1}\left(N_{g}, \bar{\chi}\right), \quad h:=\theta_{\psi_{h}} \in M_{1}\left(N_{h}, \chi\right) .
$$

Define $\psi_{1}=\psi_{g} \psi_{h}$ and $\psi_{2}=\psi_{g} \psi_{h}^{\prime}$. The characters $\psi_{1}$ and $\psi_{2}$ are ring class characters of $K$, associated to orders $\mathcal{O}_{c_{1}}$ and $\mathcal{O}_{c_{2}}$ in $\mathcal{O}_{K}$ of conductors $c_{1}$ and $c_{2}$, respectively. Such characters are sometimes also called, synonymously, dihedral or anticyclotomic characters of $K$. Note that $\psi_{g}$ and $\psi_{h}$ were not assumed to be necessarily anticyclotomic.

One checks that there is a decomposition of Artin representations and, in parallel to that, a factorization of $L$-series as follows:

$$
V_{g h}=V_{\psi_{1}} \oplus V_{\psi_{2}}, \quad L\left(E, \varrho_{g h}, s\right)=L\left(E / K, \psi_{1}, s\right) \cdot L\left(E / K, \psi_{2}, s\right) .
$$

Assume also that $\operatorname{gcd}\left(N_{f}, \mathfrak{c}_{g} \mathfrak{c}_{h}\right)=1$. This implies that, for all places of $K$ above any place $v \mid N \infty$ of $\mathbb{Q}$, the local signs of $L\left(E / K, \psi_{1}, s\right)$ and of $L(E / K$, $\left.\psi_{2}, s\right)$ are all equal, and hence $\varepsilon_{v}\left(E, \varrho_{g h}\right)=( \pm 1)^{2}=1$. This automatically implies that Hypothesis A holds.

Let $H$ denote the ring class field of conductor $c:=\operatorname{lcm}\left(c_{1}, c_{2}\right)$ of $K$, which contains the fields cut out by $\psi_{1}$ and $\psi_{2}$. The field $L$ of coefficients that is implicit in (37) can be taken to be any finite extension of the field generated by the traces of $V_{\psi_{1}}$ and $V_{\psi_{2}}$.

3.1. Heegner points and elliptic units. In the rank-(1,1) setting of Conjecture ES in which $r\left(E, V_{\psi_{1}}\right)=r\left(E, V_{\psi_{2}}\right)=1$, the global points and units that arise on the right-hand side are expected to be expressible in terms of Heegner points and elliptic units. Not surprisingly, these global constructions are the key to the theoretical evidence that has been amassed in support of Conjecture ES in the rank- $(1,1)$ setting when $p$ is split in $K$.

The Heegner point construction is available under the following hypothesis.

Heegner hypothesis: The level of $f$ may be factorized as $N_{f}=N_{f}^{+} N_{f}^{-}$, where

- there exists an integral ideal $\mathfrak{N}$ of $K$ such that $\mathcal{O}_{K} / \mathfrak{N} \simeq \mathbb{Z} / N_{f}^{+} \mathbb{Z}$; and

- $N_{f}^{-}$is the square-free product of an even number of primes which are all inert in $K$. 
This hypothesis implies that the signs which occur in the functional equations for $L\left(E, V_{\psi_{1}}, s\right)$ and $L\left(E, V_{\psi_{2}}, s\right)$ are -1 , and hence that the analytic ranks $r_{\mathrm{an}}\left(E, V_{\psi_{1}}\right)$ and $r_{\mathrm{an}}\left(E, V_{\psi_{2}}\right)$ are both odd. In particular, Hypothesis B of the introduction is automatically satisfied. It is further expected that, in 'generic' instances,

$$
r_{\text {an }}\left(E, V_{\psi_{1}}\right)=r_{\text {an }}\left(E, V_{\psi_{2}}\right)=1,
$$

and that these equalities certainly hold when the $p$-adic iterated integrals attached to the triple $(f, g, h)$ are not identically zero.

The decomposition in (37) can be further refined to

$$
\varrho_{g h}=\varrho_{1} \oplus \varrho_{2} \oplus \varrho_{3},
$$

where

- for $i=1,2, \varrho_{i}$ is the unique irreducible constituent of $V_{\psi_{i}}$ for which $r_{\mathrm{an}}\left(E, \varrho_{i}\right)$ is odd; and

- $\varrho_{3}$ is the $G_{\mathbb{Q}^{-}}$-stable complement of $\varrho_{1} \oplus \varrho_{2}$ in $\varrho_{g h}$.

This decomposition is uniquely determined by $(f, g, h)$, up to possibly switching $\varrho_{1}$ and $\varrho_{2}$. When (38) is satisfied, the decomposition (39) satisfies the conditions

$$
r_{\text {an }}\left(E, \varrho_{1}\right)=r_{\text {an }}\left(E, \varrho_{2}\right)=1, \quad r_{\text {an }}\left(E, \varrho_{3}\right)=0 .
$$

As will be explained shortly, the work of Gross, Zagier, Kolyvagin, and Zhang further shows that the same equations as in (40) hold for the corresponding algebraic ranks:

$$
r\left(E, \varrho_{1}\right)=r\left(E, \varrho_{2}\right)=1, \quad r\left(E, \varrho_{3}\right)=0,
$$

consistent with the predictions made by the Birch and Swinnerton-Dyer conjecture, and so we are in the rank- $(1,1)$ setting of Conjecture ES. It also leads to the construction of explicit nontrivial elements in $E(H)_{L}^{\varrho_{1}}$ and $E(H)_{L}^{\varrho_{2}}$ from Heegner points, which we now describe.

Let $X$ be the Shimura curve associated to an Eichler order of level $N_{f}^{+}$in the (indefinite, thanks to the Heegner hypothesis) quaternion algebra of discriminant $N_{f}^{-}$over $\mathbb{Q}$. Let

$$
\pi_{E}: \operatorname{Jac}(X) \rightarrow E
$$

denote a modular parameterization of $E$ arising from the fact that $E$ is an abelian variety quotient of the Jacobian of $X$.

Let $X(H)_{\mathcal{O}_{c}} \subset X(H)$ denote the set of Heegner points attached to the order of conductor $c$ in $K$. It is a finite set, and any two points in it are related by 
the action of an Atkin-Lehner involution and an element of $G:=\mathrm{Gal}(H / K)$. A Heegner divisor of conductor $c$ on $X$ is any degree-zero divisor supported on $X(H)_{\mathcal{O}_{c}}$, and a Heegner point on $E$ of conductor $c$ is the image of such a divisor.

After fixing a choice of $P \in X(H)_{\mathcal{O}_{c}}$, any ring class character $\psi$ of conductor $c$ gives rise to a degree-zero divisor on $X$ by setting

$$
D_{\psi}:= \begin{cases}\sum_{\sigma \in G} \psi^{-1}(\sigma) P^{\sigma} & \text { if } \psi \neq 1, \\ \sum_{\sigma \in G} P^{\sigma}-\# G \cdot \infty & \text { if } \psi=1,\end{cases}
$$

where $\infty$ is the degree-one divisor in $\operatorname{Div}(X) \otimes \mathbb{Q}$ defined by $\infty=$ $(1 /(2 g-2)) \mathcal{K}_{X}$, and $\mathcal{K}_{X}$ denotes the canonical class on $X$. The Heegner point attached to $\psi$ is then defined to be

$$
P_{\psi}:=\pi_{E}\left(D_{\psi}\right) \in E(H)_{L} .
$$

Note that the point $P_{\psi}$ actually belongs to $E(H)_{L}^{V_{\psi}}$, and that the same is true of $P_{\bar{\psi}}$.

LEMMA 3.1. Assume that (40) is satisfied. Then (41) holds as well, and, for $i=1$ and 2, the Mordell-Weil group $E(H)_{L}^{\varrho_{i}}$ is generated by the vectors $P_{\psi_{i}}$ and $P_{\bar{\psi}_{i}}$. If $\psi_{i} \neq \bar{\psi}_{i}$, these points are linearly independent, and they form a basis for $E(H)_{L}^{\varrho_{i}}$. If $\psi_{i}=\bar{\psi}_{i}$ (that is, if $\psi_{i}$ is a genus character), then $P_{\psi_{i}}=P_{\bar{\psi}_{i}}$, and this vector is a basis for $E(H)_{L}^{\varrho_{i}}$.

Proof. Assumption (40) asserts that the central critical derivative $L^{\prime}\left(E, \varrho_{i}, 1\right)$ is nonzero. The Gross-Zagier formula and its extension to ring class characters given in $[\mathbf{Z h}]$ show that the vectors $P_{\psi_{i}}$ and $P_{\bar{\psi}_{i}}$ have nonzero canonical height, and hence are nonzero in $E(H)_{L}^{V_{\psi_{i}}}$. The main theorem of [BD1] (and a suitable extension to Heegner points arising from Shimura curves as given in [YZZ]) imply that $r\left(E, V_{\psi_{i}}\right)=1$, and that $E(H)_{L}^{Q_{i}}$ is generated by the Heegner vectors $P_{\psi_{i}}$ and $P_{\bar{\psi}_{i}}$. The result follows.

Note that the points $P_{\psi_{i}}$ and $P_{\bar{\psi}_{i}}$ depend on the choice of a point $P \in X(H)_{\mathcal{O}_{c}}$ that was made in (42), but only up to multiplication by a root of unity. More precisely, replacing $P$ by $w P^{\sigma_{0}}$ for $\sigma_{0} \in G$ and $w$ a Fricke involution on $X$ has the effect of replacing $P_{\psi_{i}}$ by $\pm \bar{\psi}_{i}\left(\sigma_{0}\right) P_{\psi_{i}}$ and $P_{\bar{\psi}_{i}}$ by $\pm \psi_{i}\left(\sigma_{0}\right) P_{\bar{\psi}_{i}}$. In particular, the $L$-vector space in $E(H)_{L}$ spanned by $P_{\psi_{i}}$ is completely canonical and does not depend on the choice of $P$. 
It will also be convenient to introduce a related basis of $E(H)_{L}^{\varrho_{i}}$ attached to a choice of reflection $\tau$ in the dihedral Galois group $\operatorname{Gal}(H / \mathbb{Q})$, by setting

$$
P_{\psi_{i}}^{+}=P_{\psi_{i}}+P_{\psi_{i}}^{\tau}, \quad P_{\psi_{i}}^{-}=P_{\psi_{i}}-P_{\psi_{i}}^{\tau} .
$$

Sometimes, the basic Heegner point $P$ used to define the points $P_{\psi_{i}}^{ \pm}$can be chosen to be fixed (up to sign) by the reflection $\tau$, that is, in such a way that $\tau P= \pm P$. When this is the case,

$$
P_{\psi_{i}}^{+}=P_{\psi_{i}} \pm P_{\bar{\psi}_{i}}, \quad P_{\psi_{i}}^{-}=P_{\psi_{i}} \mp P_{\bar{\psi}_{i}} .
$$

But this simpler expression for the basis $\left(P_{\psi_{i}}^{+}, P_{\psi_{i}}^{-}\right)$need not be invoked in general.

Let

$$
\psi_{0}:=\psi_{g} / \psi_{g}^{\prime}
$$

be the nontrivial ring class character attached to the adjoint representation of $g$. In addition to the Heegner points $P_{\psi_{i}}$ and $P_{\psi_{i}}^{ \pm}$, Conjecture ES also involves the elliptic unit $u \in \mathcal{O}_{H_{g}}^{\times}$and its $\psi_{0}$-component

$$
u_{\psi_{0}}=\sum_{\sigma \in G} \psi_{0}^{-1}(\sigma) u^{\sigma} \in\left(\mathcal{O}_{H_{g}}^{\times}\right)_{L}^{V_{\psi_{0}}},
$$

where the group law in the unit group $\mathcal{O}_{H_{G}}^{\times}$is written additively, and the units $u_{\psi_{0}}^{ \pm}$relative to any choice of a reflection $\tau \in \operatorname{Gal}(H / \mathbb{Q})$ are defined just as in (43):

$$
u_{\psi_{0}}^{+}=u_{\psi_{0}}+u_{\psi_{0}}^{\tau}, \quad u_{\psi_{0}}^{-}=u_{\psi_{0}}-u_{\psi_{0}}^{\tau} .
$$

Note that, just as for the points $P_{\psi_{i}}$ and $P_{\psi_{i}}^{ \pm}$, the group $\operatorname{Gal}(H / K)$ acts on the element $u_{\psi_{0}}$ through the character $\psi_{0}$, but does not preserve the line spanned by either $u_{\psi_{0}}^{+}$or $u_{\psi_{0}}^{-}$.

3.2. Conjecture ES when $\boldsymbol{p}$ splits in $\boldsymbol{K}$. When $p$ is split in $K$, the Frobenius element $\sigma_{p}$ belongs to $\operatorname{Gal}(H / K)$, and the choice of a prime $\wp$ of $K$ above $p$ is sufficient to determine $\sigma_{p}$ as an element of this group. The choices of $\alpha_{g}, \beta_{g}, \alpha_{h}$, and $\beta_{h}$ can then be made so that

$$
\alpha_{g}=\psi_{g}(\wp), \quad \beta_{g}=\psi_{g}(\bar{\wp}), \quad \alpha_{h}=\psi_{h}(\wp), \quad \beta_{h}=\psi_{h}(\bar{\wp}) .
$$

With the above choices,

$$
\alpha_{g} \alpha_{h}=\psi_{g} \psi_{h}(\wp)=\psi_{1}\left(\sigma_{p}\right), \quad \alpha_{g} \beta_{h}=\psi_{g} \psi_{h}^{\prime}(\wp)=\psi_{2}\left(\sigma_{p}\right) .
$$

The following lemma describes the $p$-adic regulator $\operatorname{det} R_{g_{\alpha}}\left(E, \varrho_{g h}\right)$ that enters into Conjecture ES. 
LEMMA 3.2. The regulator $\operatorname{det}\left(R_{g_{\alpha}}\left(E, \varrho_{g h}\right)\right)$ is given by the following expressions.

(1) If $r\left(E, V_{\psi_{1}}\right)=r\left(E, V_{\psi_{2}}\right)=1$,

$$
\operatorname{det} R_{g_{\alpha}}\left(E, \varrho_{g h}\right)=\log _{E, p}\left(P_{\psi_{1}}\right) \cdot \log _{E, p}\left(P_{\psi_{2}}\right) \text {. }
$$

(2) If $r\left(E, V_{\psi_{1}}\right) \geqslant 2 \operatorname{orr}\left(E, V_{\psi_{2}}\right) \geqslant 2$,

$$
\operatorname{det} R_{g_{\alpha}}\left(E, \varrho_{g h}\right)=0 .
$$

Proof. The first part follows from the fact that, after choosing a $\sigma_{p}$-eigenbasis for $V_{g h}^{g_{\alpha}}$, the matrix $R_{g_{\alpha}}\left(E, \varrho_{g h}\right)$ takes the form

$$
R_{g_{\alpha}}\left(E, \varrho_{g h}\right)=\left(\begin{array}{cc}
\log _{E, p}\left(P_{\psi_{1}}\right) & 0 \\
0 & \log _{E, p}\left(P_{\psi_{2}}\right)
\end{array}\right)
$$

In the rank-2 setting, assume without loss of generality that $r\left(E, V_{\psi_{1}}\right)=2$ and that $r\left(E, V_{\psi_{2}}\right)=0$. The two-dimensional subspace $V_{g h}^{g \alpha}$ then intersects $V_{\psi_{1}}$ and $V_{\psi_{2}}$ in one-dimensional subspaces which determine a decomposition of $V_{g h}^{g_{\alpha}}$. Choosing a basis $\left(v_{1}, v_{2}\right)$ for $V_{g h}^{g_{\alpha}}$ which is compatible with this decomposition, and a basis $\Phi_{1}, \Phi_{2}$ of $\operatorname{Hom}_{G_{\mathbb{Q}}}\left(V_{\psi_{1}}, E(H)_{L}\right)$, produces a matrix $R_{g_{\alpha}}\left(E, \varrho_{g h}\right)$ whose second column is zero.

The lemma above motivates focusing our attention on the rank- $(1,1)$ setting of Conjecture ES when $p$ is split, which is what shall be done for the remainder of Section 3.2. Assume as in Section 3.1 that the Heegner hypothesis is satisfied, and let

$$
P_{1}:=P_{\psi_{1}}, \quad P_{2}:=P_{\psi_{2}}
$$

be the Heegner points constructed in the previous section. The main result of this section is the following.

THEOREM 3.3. Assume that $N=\operatorname{lcm}\left(N_{f}, N_{g}, N_{h}\right)$ is square free and that $p \nmid 2 N$. Assume also that Hypotheses $\mathrm{C}$ and $\mathrm{C}^{\prime}$ hold. If $r_{\mathrm{an}}\left(E, V_{\psi_{1}}\right)>1$ or $r_{\mathrm{an}}\left(E, V_{\psi_{2}}\right)>1$, the iterated integrals attached to the triple $(f, g, h)$ vanish identically. Otherwise, there exist a finite extension $L$ of $\mathbb{Q}\left(\psi_{g}, \psi_{h}\right)$, a scalar $\lambda \in L^{\times}$, and test vectors

$$
\left(\breve{f}, \breve{\gamma}_{g_{\alpha}}, \breve{h}\right) \in S_{2}(N)_{L}[f] \times M_{1}(N p, \chi)_{L}^{\vee}\left[g_{\alpha}\right] \times M_{1}(N, \chi)_{L}[h]
$$

for which

$$
\int_{\breve{\gamma}_{g \alpha}} \breve{f} \cdot \breve{h}=\lambda \cdot \frac{\log _{E, p}\left(P_{1}\right) \log _{E, p}\left(P_{2}\right)}{\log _{p}\left(u_{g_{\alpha}}\right)} .
$$


REMARK 3.4. We believe that in fact $L$ can always be taken to be $\mathbb{Q}\left(\psi_{g}, \psi_{h}\right)$. It would be interesting to be able to pin down a concrete choice of test vectors $\left(\breve{f}, \breve{\gamma}_{g_{\alpha}}, \breve{h}\right)$ for which the precise value of $\lambda \in L^{\times}$could be computed explicitly. For example, let $\chi_{K}$ denote a quadratic Dirichlet character of prime conductor $D_{K}$ associated to an imaginary quadratic field $K$. Assume that $N=D_{K}$ and that $\psi_{g}=\psi_{h}=1$, so that

$$
g=h=\theta_{1} \in M_{1}\left(D_{K}, \chi_{K}\right)
$$

is the Eisenstein theta series associated to the trivial character of $K$. Since $N_{f}=$ $N_{g}=N_{h}=N$, a natural choice of test vectors, which is unique up to scaling by elements in $L^{\times}$, is simply $\left(f, \gamma_{g_{\alpha}}, h\right)$. The following explicit formula has been verified experimentally in a number of examples, and should be amenable to a proof by the methods of this section:

$$
\int_{\gamma_{g \alpha}} f \cdot h=\frac{\left|E\left(\mathbb{F}_{p}\right)\right|^{2} \log _{E, p}\left(P_{K}\right)^{2}}{p(p-1) h_{K} \log _{p}\left(u_{\wp}\right)} .
$$

Here, $\left|E\left(\mathbb{F}_{p}\right)\right|$ is the number of points on $E$ over the finite field $\mathbb{F}_{p}, h_{K}$ is the class number of $K, P_{K}$ is the Heegner point in $E(K)$, and $u_{\wp} \in K^{\times}$is any $p$-unit satisfying $\left(u_{\wp}\right)=\wp^{h_{K}}$.

Formula (45) was first discovered experimentally by the authors (on Christmas Day), after a long theoretical and experimental investigation trying to uncover the arithmetic meaning of these $p$-adic iterated integrals. It is the prototype for all the formulae in this paper.

The remainder of this section is devoted to proving Theorem 3.3. The proof rests on a comparison between the Garrett-Hida $p$-adic $L$-function of the previous section and two different types of 'Katz-style' $p$-adic $L$-functions whose definitions are recalled below.

(1) The Katz two-variable p-adic L-function of an imaginary quadratic field.

Let $\mathfrak{c} \subseteq \mathcal{O}_{K}$ be an integral ideal of the imaginary quadratic field $K$, and denote by $\Sigma$ the set of Hecke characters of $K$ of conductor dividing $\mathfrak{c}$. Define $\Sigma_{K}=$ $\Sigma_{K}^{(1)} \cup \Sigma_{K}^{(2)} \subset \Sigma$ to be the disjoint union of the sets

$$
\begin{aligned}
& \Sigma_{K}^{(1)}=\left\{\psi \in \Sigma \text { of infinity type }\left(\kappa_{1}, \kappa_{2}\right), \text { with } \kappa_{1} \leqslant 0, \kappa_{2} \geqslant 1\right\}, \\
& \Sigma_{K}^{(2)}=\left\{\psi \in \Sigma \text { of infinity type }\left(\kappa_{1}, \kappa_{2}\right), \text { with } \kappa_{1} \geqslant 1, \kappa_{2} \leqslant 0\right\} .
\end{aligned}
$$

For all $\psi \in \Sigma_{K}$, the complex argument $s=0$ is a critical point for the Hecke $L$ function $L\left(\psi^{-1}, s\right)$, and Katz's $p$-adic $L$-function is constructed by interpolating the (suitably normalized) values $L\left(\psi^{-1}, 0\right)$ as $\psi$ ranges over $\Sigma_{K}^{(2)}$. 
More precisely, let $\hat{\Sigma}_{K}$ denote the completion of $\Sigma_{K}^{(2)}$ with respect to the compact open topology on the space of $\mathcal{O}_{L_{p}}$-valued functions on a certain subset of $\mathbf{A}_{K}^{\times}$, as described in [BDP2, Section 5.2]. By the work of Katz [Katz1], there exists a $p$-adic analytic function

$$
L_{p}(K): \hat{\Sigma}_{K} \longrightarrow \mathbb{C}_{p}
$$

which is uniquely characterized by the following interpolation property: for all $\psi \in \Sigma_{K}^{(2)}$ of infinity type $\left(\kappa_{1}, \kappa_{2}\right)$,

$$
L_{p}(K)(\psi)=\mathfrak{a}(\psi) \times \mathfrak{e}(\psi) \times \mathfrak{f}(\psi) \times \frac{\Omega_{p}^{\kappa_{1}-\kappa_{2}}}{\Omega^{\kappa_{1}-\kappa_{2}}} \times L_{c}\left(\psi^{-1}, 0\right),
$$

where

- $\mathfrak{a}(\psi)=\left(\kappa_{1}-1\right) ! \pi^{-\kappa_{2}}, \mathfrak{e}(\psi)=\left(1-\psi(\wp) p^{-1}\right)\left(1-\psi^{-1}(\bar{\wp})\right)$, and $\mathfrak{f}(\psi)=$ $D_{K}^{\kappa_{2} / 2} 2^{-\kappa_{2}}$

- $\Omega_{p} \in \mathbb{C}_{p}^{\times}$is a $p$-adic period attached to $K$, as defined in [BDP1, (2-17)];

- $\Omega \in \mathbb{C}^{\times}$is the complex period associated to $K$ defined in [BDP1, (2-15)]; and

- $L_{c}\left(\psi^{-1}, s\right)$ is Hecke's $L$-function associated to $\psi^{-1}$ with the Euler factors at primes dividing $c$ removed.

Formula (46) is [BDP1, Proposition 3.1], which in turn follows from [Katz2, Section 5.3.0] (see also [Gro, Theorem 2.3] and [deS, II, Theorem 4.14]; the reader is cautioned that the latter reference needs to be slightly modified, for the power of $2 \pi$ is not correct there).

Letting $\mathbf{N}_{K}$ denote the norm of $K / \mathbb{Q}$ regarded as a Hecke character of $K$ of infinity type $(1,1)$, Katz's $p$-adic $L$-function satisfies the functional equation $L_{p}(K)(\psi)=L_{p}(K)\left(\left(\psi^{\prime}\right)^{-1} \mathbf{N}_{K}\right)$ (see [Gro, page 90-91]).

The values of $p$-adic $L$-functions at classical points lying outside their region of interpolation are frequently of great arithmetic interest, since they may encode further invariants of the associated motives. An instance of this philosophy is Katz's $p$-adic analogue of the Kronecker limit formula (see [Katz1, Section 10.4, 10.5], [Gro, pages 90-91], [deS, Chapter II, Section 5.2]), expressing the value of $L_{p}(K)$ at a finite order character $\psi$ of $G_{K}$ in terms of a suitable linear 
combination of $p$-adic logarithms of elliptic units:

$$
L_{p}(K)(\psi)= \begin{cases}\frac{1}{2}\left(\frac{1}{p}-1\right) \cdot \log _{p}\left(u_{\wp}\right) & \text { if } \psi=1, \\ \frac{-1}{24 c}\left(1-\psi^{-1}(\bar{\wp})\right)\left(1-\psi(\wp) p^{-1}\right) & \\ \cdot \sum_{\sigma \in G} \psi^{-1}(\sigma) \log _{p}(\sigma(u)) & \text { if } \psi \neq 1 .\end{cases}
$$

Here, as above, $u_{\wp} \in K^{\times}$is any element satisfying $\left(u_{\wp}\right)=\wp^{h_{K}}$, and $u \in \mathcal{U}_{\mathrm{CM}}$ is an elliptic unit; the integer $c>0$ is the smallest positive integer in the conductor ideal of $\psi$.

(2) The p-adic Rankin L-function attached to a cusp form and an imaginary quadratic field. For any Hecke character $\psi$ of $K$ of infinity type $\left(\kappa_{1}, \kappa_{2}\right)$, let

$$
L(f, \psi, s):=L\left(\pi_{f} \times \pi_{\psi}, s-\frac{\kappa_{1}+\kappa_{2}+1}{2}\right)
$$

denote the $L$-series associated with the product of the global automorphic representations attached to the weight-two cusp form $f$ and the Hecke character $\psi$, normalized conveniently. As usual, $L(f, \psi, s)=\prod_{q} L_{(q)}\left(q^{-s}\right)$ is defined as a product of Euler factors ranging over the set of prime numbers.

Fix a positive integer $c \geqslant 1$ which is relatively prime to $p N_{f}$. Let $\Sigma_{f, c}$ be the set of Hecke characters $\psi \in \Sigma$ of conductor $c$ and trivial central character for which $L\left(f, \psi^{-1}, s\right)$ is self-dual and has $s=0$ as its central critical point. This set can be expressed as the disjoint union of the three subsets

$$
\begin{gathered}
\Sigma_{f, c}^{(1)}=\left\{\psi \in \Sigma_{f, c} \text { of infinity type }(1,1)\right\}, \\
\Sigma_{f, c}^{(2)}=\left\{\psi \in \Sigma_{f, c} \text { of infinity type }(\kappa+2,-\kappa), \kappa \geqslant 0\right\},
\end{gathered}
$$

and

$$
\Sigma_{f, c}^{\left(2^{\prime}\right)}=\left\{\psi \in \Sigma_{f, c} \text { of infinity type }(-\kappa, \kappa+2), \kappa \geqslant 0\right\} .
$$

The three sets $\Sigma_{f, c}^{(1)}, \Sigma_{f, c}^{(2)}$, and $\Sigma_{f, c}^{\left(2^{\prime}\right)}$ are each dense in the completion $\hat{\Sigma}_{f, c}$ of $\Sigma_{f, c}$ with respect to the $p$-adic compact open topology as explained in [BDP2, Section 5.2]. As shown in loc. cit., there exists a unique $p$-adic analytic function

$$
L_{p}(f, K): \hat{\Sigma}_{f, c} \longrightarrow \mathbb{C}_{p}
$$

interpolating the critical values $L\left(f, \psi^{-1}, 0\right)$ for $\psi \in \Sigma_{f, c}^{(2)}$, suitably normalized. The function $L_{p}(f, K)$ is referred to as the $p$-adic Rankin $L$-function attached 
to the pair $(f, K)$. By [BDP1, Proposition 3.11], the interpolation formula reads precisely as follows: for any character $\psi \in \Sigma_{f, c}^{(2)}$ of infinity type $(\kappa+2,-\kappa)$,

$$
L_{p}(f, K)(\psi)=\mathfrak{a}(f, \psi) \times \mathfrak{e}^{2}(f, \psi) \times \mathfrak{f}(f, \psi) \times \frac{\Omega_{p}^{4 \kappa+4}}{\Omega^{4 \kappa+4}} \times L\left(f, \psi^{-1}, 0\right),
$$

where

- $\mathfrak{a}(f, \psi)=\kappa !(\kappa+1) ! \pi^{2 \kappa+1}, \mathfrak{e}(f, \psi)=1-a_{p}(f) \psi^{-1}(\bar{\wp})+\psi^{-2}(\bar{\wp}) p$, and

- $\mathfrak{f}(f, \psi)=\left(2 / c \sqrt{D_{K}}\right)^{2 \kappa+1} \cdot \prod_{q \mid c}\left(q-\chi_{K}(q)\right) /(q-1) \cdot \omega(f, \psi)^{-1}$.

Here, $\omega(f, \psi)$ denotes the complex number defined in [BDP2, (5.1.11)]. It is an algebraic scalar of absolute value 1 relative to any complex embedding of $\overline{\mathbb{Q}}$.

Consider now a Hecke character $\psi \mathbf{N}_{K} \in \Sigma_{f, c}^{(1)}$, where $\psi$ has finite order. The character $\psi \mathbf{N}_{K}$ lies outside the region of interpolation of $L_{p}(f, K)$, and the main theorem of [BDP2] - see [Br] for a proof of it under the more relaxed Heegner hypothesis imposed here-asserts that

$$
L_{p}(f, K)\left(\psi \mathbf{N}_{K}\right)=\left(1-\frac{a_{p}(f)}{\psi(\bar{\wp}) p}+\frac{1}{\psi^{2}(\bar{\wp}) p}\right)^{2} \times \log _{\omega_{E}}\left(P_{\psi}\right)^{2} .
$$

We now turn to the comparison of the Rankin $p$-adic $L$-function with the Garrett-Hida $p$-adic $L$-function of the previous section: the Katz $p$-adic $L$ function then arises indirectly from the ratio of periods (51) used in constructing these two $p$-adic $L$-functions.

Recall the theta series $g$ of weight one, level $N_{g}=D_{K} N_{K / \mathbb{Q}}\left(\mathfrak{c}\left(\psi_{g}\right)\right)$, and character $\chi^{-1}$ appearing in the statement of Theorem 3.3.

Let $\mathbf{g}$ be the primitive cuspidal Hida family of theta series of tame level $N_{g}$ and tame character $\chi_{g}=\chi^{-1}$ constructed in for example [Hi2, pages 235-236] and [Gh, Section 5], specializing in weight one to the $p$-stabilization $g_{\alpha}$ of $g$ satisfying $U_{p}\left(g_{\alpha}\right)=\alpha_{g} \cdot g_{\alpha}$. It can be described explicitly by fixing a Hecke character $\lambda$ of infinity type $(1,0)$ and conductor $\wp$. Let $\mathbb{Q}(\lambda)$ denote the number field generated by the values of $\lambda$, and let $\mathbb{Q}_{p}(\lambda)$ be the completion of $\mathbb{Q}(\lambda)$ at the prime determined by an embedding $\mathbb{Q}(\lambda) \hookrightarrow \overline{\mathbb{Q}}_{p}$ which is fixed at the outset. Writing $\mathcal{O}_{\mathbb{Q}_{p}(\lambda)}$ for its ring of integers, decompose its group of units as $\mathcal{O}_{\mathbb{Q}_{p}(\lambda)}^{\times}=\mu \times W$, where $\mu$ is finite and $W$ is free over $\mathbb{Z}_{p}$. Let $\langle\cdot\rangle: \mathcal{O}_{\mathbb{Q}_{p}(\lambda)}^{\times} \rightarrow W$ denote the natural projection.

For every integer $k \geqslant 1$, define

$$
\psi_{g, k-1}^{(p)}=\psi_{g}\langle\lambda\rangle^{k-1} .
$$

This definition does not depend on the choice of $\lambda$, for any other such choice $\lambda^{\prime}$ differs from $\lambda$ by a finite character, and hence $\left\langle\lambda^{\prime}\right\rangle=\langle\lambda\rangle$. Let now $\psi_{g, k-1}$ be 
the Hecke character defined by

$$
\psi_{g, k-1}(\mathfrak{q})= \begin{cases}\psi_{g, k-1}^{(p)}(\mathfrak{q}) & \text { if } \mathfrak{q} \neq \wp, \\ \chi^{-1}(p) p^{k-1} / \psi_{g, k-1}^{(p)}(\bar{\wp}) & \text { if } \mathfrak{q}=\wp .\end{cases}
$$

The Hecke character $\psi_{g, k-1}$ has conductor $\mathfrak{c}\left(\psi_{g}\right)$ and infinity type $(k-1,0)$. For any $k \in \mathbb{Z}_{\geqslant 1} \cap \mathcal{U}_{g}$, the weight $k$ specialization of $\mathbf{g}$ is the ordinary $p$ stabilization of the theta series $g_{k}=\theta_{\psi_{g, k-1}}=\theta_{\psi_{g, k-1}^{\prime}}$ associated with $\psi_{g, k-1}$ (or with its $\mathrm{Gal}(K / \mathbb{Q})$-conjugate $\left.\psi_{g, k-1}^{\prime}\right)$. In particular, $\psi_{g, 0}=\psi_{g}$ is the character we considered at the outset, and $g_{1}=g$.

Together with $g$, recall also the eigenforms $f \in S_{2}\left(N_{f}\right)$ and $h \in M_{1}\left(N_{h}, \chi\right)$ of the statement. Let $(\breve{g}, \breve{f}, \breve{h})$ be Watson-Woodbury's test vector of level $N$ associated to these three modular forms as described in Remark 2.2, and let also $\breve{\mathbf{g}}$ denote Watson-Woodbury's $\Lambda$-adic test vector discussed in Remark 2.4. Recall that, as discussed in Section $2, W_{v}=C_{v}\left(\breve{g}_{k}, \breve{f}, \breve{h}\right) \in \mathbb{Q}^{\times}$are constants independent of $k$ for all $v \mid N$. Moreover, the results of [Wo2] guarantee that in this setting $C_{\infty}\left(\breve{g}_{k}, \breve{f}, \breve{h}\right)=1$ for all $k$.

Recall the triple-product $p$-adic $L$-functions introduced in the previous section. Denote by $k \mapsto \mathscr{L}_{p}^{g}\left(\breve{\mathbf{g}}, \breve{f}_{2}, \breve{h}_{1}\right)(k)$ the restriction of $\mathscr{L}_{p}^{g}(\breve{\mathbf{g}}, \breve{\mathbf{f}}, \breve{\mathbf{h}})$ to $\mathcal{U}_{g} \times\{2\} \times\{1\}$. Because it does not involve the variation of $f_{2}$ in a Hida family, this restriction has the virtue of being defined a bit more generally, without any ordinariness assumptions on $f_{2}$.

Proposition 2.3 implies, for all $k \in \mathcal{U}_{g}^{\circ}$, that

$$
\begin{aligned}
& \mathscr{L}_{p}^{g}(\breve{\mathbf{g}}, \breve{f}, \breve{h})(k) \cdot \mathscr{L}_{p}^{g}\left(\breve{\mathbf{g}}^{*}, \breve{f}, \breve{h}^{*}\right)(k) \\
& \quad=\mathfrak{e}(k, 2,1)^{2} \cdot \mathfrak{f}(k, 2,1) \cdot \mathfrak{a}(k, 2,1) \cdot \prod_{v \mid N} W_{v} \cdot \frac{L\left(g_{k} \otimes f \otimes h, \frac{k+1}{2}\right)}{\left\langle g_{k}^{*}, g_{k}^{*}\right\rangle^{2}} .
\end{aligned}
$$

Here, the factors on the right are described in Proposition 2.3. In particular,

$$
\mathfrak{f}(k, 2,1)=A \cdot 2^{B} \quad \text { and } \quad \mathfrak{a}(k, 2,1)=\left(\left(\frac{k-3}{2}\right) !\left(\frac{k-1}{2}\right) !\right)^{2} \cdot \pi^{-2 k}
$$

For every integer $k \geqslant 1$, define the Hecke characters

$$
\Psi_{g h}(k)=\left(\psi_{g, k-1}^{\prime}\right)^{-1} \cdot \psi_{h}^{-1} \cdot \mathbf{N}_{K}^{(k+1) / 2}
$$

and

$$
\Psi_{g h^{\prime}}(k)=\left(\psi_{g, k-1}^{\prime}\right)^{-1} \cdot\left(\psi_{h}^{\prime}\right)^{-1} \cdot \mathbf{N}_{K}^{(k+1) / 2} .
$$

While for $k=1$ the characters $\Psi_{g h}(1)=\mathbf{N}_{K} \cdot\left(\psi_{g}^{\prime} \psi_{h}\right)^{-1}$ and $\Psi_{g h^{\prime}}(1)=\mathbf{N}_{K}$. $\left(\psi_{g}^{\prime} \psi_{h}^{\prime}\right)^{-1}$ lie in $\Sigma_{f, c}^{(1)}$, for weights $k \in \mathcal{U}_{g}^{\circ}, k>1$, the characters $\Psi_{g h}(k), \Psi_{g h^{\prime}}(k)$ 
belong to $\Sigma_{f, c}^{(2)}$ with $c=\operatorname{lcm}\left(c_{1}, c_{2}\right)$, because their infinity type is $(\kappa+2,-\kappa)$ with $\kappa=(k-3) / 2 \geqslant 0$.

It follows that, for weights $k \in \mathcal{U}_{g}^{\circ}, k>1$, the values $L_{p}(f, K)\left(\Psi_{g h}(k)\right)$, $L_{p}(f, K)\left(\Psi_{g h^{\prime}}(k)\right)$ and $\mathscr{L}_{p}^{g}(\breve{\mathbf{g}}, \breve{f}, \breve{h})(k)$ all lie in the region of interpolation. An inspection of the Euler factors defining their classical counterparts yields the following relationship between critical values: for every $k \in U_{g}$ there is a fudge factor $\mathfrak{f}_{0}(k) \neq 0$ such that

$$
L\left(g_{k} \otimes f \otimes h, \frac{k+1}{2}\right)=\mathfrak{f}_{0}(k) \cdot L\left(f, \Psi_{g h}^{-1}(k), 0\right) \cdot L\left(f, \Psi_{g h^{\prime}}^{-1}(k), 0\right),
$$

and we shall prove the theorem by letting $k \in \mathcal{U}_{g}^{\circ} \cap \mathbb{Z}_{>1}$ in (50) tend $p$-adically to 1 within $\mathcal{U}_{g}$, so that the triplets $(k, 2,1)$ tend to $(1,2,1)$.

Several of the fudge factors that arise in comparing different $L$-values, viewed as functions of weight space, satisfy the property described in the following definition.

DEFINITION 3.5. Let $F$ be a number field. A function

$$
\mathfrak{f}: \mathcal{U}_{g}^{\circ} \cap \mathbb{Z}_{>1} \longrightarrow \overline{\mathbb{Q}}
$$

is $F$-admissible if it extends to an element of the fraction field $\mathcal{L}_{g}$ of the algebra $\Lambda_{g}=\mathcal{O}\left(\mathcal{U}_{g}\right)$ of Iwasawa functions on $\mathcal{U}_{g}$, and if $\mathfrak{f}(1)$ is well defined and belongs to $F^{\times}$.

As in the statement of the definition, an $F$-admissible function $\mathfrak{f}$ will customarily be identified with the function on $\mathcal{U}_{g} \subseteq \mathbb{Z}_{p}$ which it induces. In order to make the calculations described below easier to follow, vowels will generally be used to denote nonadmissible functions (such as rational functions of $p^{k}, \pi^{k}$ or $k$ !), and consonants to denote admissible ones (such as $q^{k}$ or $a_{q}\left(g_{k}\right)$ for some prime number $q \neq p$ ).

Instances of admissible functions were already encountered in the proof of Proposition 2.6: using the notation employed there, the functions $\lambda_{d}$ were shown in loc. cit. to be $\mathbb{Q}\left(g_{1}, h_{1}\right)$-admissible. Recall also that the fudge factor $\mathfrak{f}_{0}(k)$ appearing in (50) accounts for the discrepancy of the Euler factors at the primes $q \mid c D_{K} N_{f}$ in the definitions of the above $L$-series. It may be computed explicitly, but we are content to note here that it is $\mathbb{Q}\left(\psi_{g}, \psi_{h}\right)$-admissible because $\mathfrak{f}_{0}(k)$ is a rational function on $q^{k}$ for $q \mid c D_{K} N_{f}$ with coefficients in $\mathbb{Q}\left(\psi_{g}, \psi_{h}\right)$, with $\mathfrak{f}_{0}(k) \neq 0$ for all $k \in \mathbb{Z}$.

For every $k \in \mathcal{U}_{g}^{\circ}, k>1$, define

$$
\sigma(k):=\left\langle g_{k}^{*}, g_{k}^{*}\right\rangle \times\left(\frac{\pi \Omega_{p}}{\Omega}\right)^{2 k-2}
$$


and

$$
\mathfrak{e}_{1}(k)=\frac{\mathfrak{e}(k, 2,1)^{2}}{\mathfrak{e}^{2}\left(f, \Psi_{g h}(k)\right) \mathfrak{e}^{2}\left(f, \Psi_{g h^{\prime}}(k)\right)}, \quad \mathfrak{f}_{1}(k)=\frac{\prod_{v \mid N} W_{v} \cdot \mathfrak{f}_{0}(k) \cdot \mathfrak{f}(k, 2,1)}{\mathfrak{f}\left(f, \Psi_{g h}(k)\right) \mathfrak{f}\left(f, \Psi_{g h^{\prime}}(k)\right)} .
$$

LEMma 3.6. The function $\mathfrak{f}_{1}(k)$ is $K\left(\psi_{g}, \psi_{h}\right)$-admissible, and

$$
\begin{aligned}
& \mathscr{L}_{p}^{g}(\breve{\mathbf{g}}, \breve{f}, \breve{h})(k) \cdot \mathscr{L}_{p}^{g}\left(\breve{\mathbf{g}}^{*}, \breve{f}, \breve{h}^{*}\right)(k) \cdot \sigma(k)^{2} \\
& \quad=\mathfrak{e}_{1}(k) \mathfrak{f}_{1}(k) \cdot L_{p}(f, K)\left(\Psi_{g h}(k)\right) \cdot L_{p}(f, K)\left(\Psi_{g h^{\prime}}(k)\right) .
\end{aligned}
$$

Proof. We already saw that $\mathfrak{f}_{0}(k)$ is $\mathbb{Q}\left(\psi_{g}, \psi_{h}\right)$-admissible, and Proposition 2.1(b)(ii) implies that $\mathfrak{f}(k, 2,1)=A \cdot 2^{B(k)}$ is $\mathbb{Q}$-admissible because $p \neq 2$ and $B(k)$ is a linear function of $k$. It remains to prove that the denominator of $\mathfrak{f}_{1}(k)$ is $K\left(\psi_{g}, \psi_{h}\right)$-admissible. Recall from (48) that $\mathfrak{f}(f, \psi)=$ $\left(2 / c \sqrt{D_{K}}\right)^{k-2} \cdot \prod_{q \mid c}\left(q-\chi_{K}(q)\right) /(q-1) \cdot \omega(f, \psi)^{-1}$ for any Hecke character $\psi \in \Sigma_{f, c}^{(2)}$ of infinity type $((k-3) / 2+2,-(k-3) / 2)$. The first two factors are $K$-admissible, because $p$ is assumed not to divide $2 N$. Hence, in order to conclude, we need to show that the functions $\omega\left(f, \Psi_{g h}(k)\right)$ and $\omega\left(f, \Psi_{g h^{\prime}}(k)\right)$ are admissible, or at least that their product is. By [BDP2, (5.1.11)],

$$
\omega\left(f, \Psi_{g h}(k)\right) \cdot \omega\left(f, \Psi_{g h^{\prime}}(k)\right)=\frac{\mathbf{N}_{K}^{2 k-2}(\mathfrak{b}) N^{k-1}}{\left(\psi_{g, k-1}^{\prime}\right)^{2} \psi_{h} \psi_{h}^{\prime}(\mathfrak{b}) \cdot b^{2 k-2}},
$$

where $(\mathfrak{b}, p N c)=1$ and $\mathfrak{b} \cdot \mathfrak{N}=(b)$. This shows that the function in (52) is $K\left(\psi_{g}, \psi_{h}\right)$-admissible, because $p \nmid \mathfrak{b} \mathfrak{N}$, and its value at $k=1$ is the nonzero algebraic number $\left(\left(\psi_{g}^{\prime}\right)^{2} \psi_{h} \psi_{h}^{\prime}(\mathfrak{b})\right)^{-1}=\left(\psi_{g}^{\prime}\right)^{2} \psi_{h} \psi_{h}^{\prime}(\mathfrak{N})$, which does not depend on the choice of $\mathfrak{b}$.

Finally, the factorization formula of Lemma 3.6 is a direct consequence of the interpolating formulae (33) and (48), combined with (50), once one observes that the factorial terms and powers of $\pi$ on both sides cancel out.

At this point it is convenient to invoke the following classical formula.

LEMMA 3.7. There is a $K$-admissible function $\mathfrak{f}_{2}(k)$ such that

$$
\left\langle g_{k}^{*}, g_{k}^{*}\right\rangle=\frac{(k-1) !}{\pi^{k}} \cdot \mathfrak{f}_{2}(k) \cdot L\left(\psi_{g, k-1}^{2} \cdot \chi, k\right) .
$$

Proof. Since the $L$-series of the adjoint of a CM-form factors as

$$
L\left(\operatorname{Ad}\left(g_{k}^{*}\right), s\right)=L\left(\operatorname{Ad}\left(g_{k}\right), s\right)=L\left(\chi_{K}, s-k+1\right) L\left(\psi_{g, k-1}^{2} \cdot \chi_{g}^{-1}, s\right)
$$


and $\chi_{g}=\chi^{-1}$, Lemma 3.7 follows directly from (27) after evaluating at $s=k$ and applying Dirichlet's class number formula $L\left(\chi_{K}, 1\right)=2 \pi h_{K}\left(\left|\mathcal{O}_{K}^{\times}\right| \sqrt{D}\right)^{-1}$.

It now becomes clear how Katz's $p$-adic $L$-function enters in the calculation: note that

$$
L\left(\psi_{g, k-1}^{2} \cdot \chi, k\right)=\mathfrak{f}_{3}(k) \cdot L_{c}\left(\psi_{g, k-1}^{2} \cdot \chi \cdot \mathbf{N}_{K}^{-k}, 0\right),
$$

where again $\mathfrak{f}_{3}(k)$ accounts for the discrepancy between the Euler factors at primes $q \mid c D_{K}$ defining both $L$-series above, and hence is $\mathbb{Q}\left(\psi_{g}, \psi_{h}\right)$ admissible.

Define

$$
\Psi_{g}(k):=\left(\psi_{g, k-1}^{\prime}\right)^{-2} \chi^{-1} \mathbf{N}_{K}^{k} .
$$

LEMMA 3.8. The following equality holds for every $k \in \mathcal{U}_{g}^{\circ}, k>1$ :

$$
L_{p}(K)\left(\Psi_{g}(k)\right)=\frac{\mathfrak{e}\left(\Psi_{g}(k)\right) \cdot \mathfrak{f}\left(\Psi_{g}(k)\right)}{\mathfrak{f}_{2}(k) \cdot \mathfrak{f}_{3}(k)} \times\left\langle g_{k}^{*}, g_{k}^{*}\right\rangle \times\left(\frac{\pi \Omega_{p}}{\Omega}\right)^{2 k-2}
$$

Proof. In light of Katz's interpolation formula (46), since the infinity type of $\Psi_{g}(k)$ is $\left(\kappa_{1}, \kappa_{2}\right)=(k,-k+2)$, it follows that

$$
\begin{aligned}
L\left(\psi_{g, k-1}^{2} \cdot \chi, k\right) & =L\left(\left(\psi_{g, k-1}^{\prime}\right)^{2} \cdot \chi, k\right) \\
& =\frac{\pi^{-k+2}}{(k-1) !} \cdot \frac{1}{\mathfrak{e}\left(\Psi_{g}(k)\right)} \cdot \frac{\mathfrak{f}_{3}(k)}{\mathfrak{f}\left(\Psi_{g}(k)\right)} \cdot \frac{\Omega^{2 k-2}}{\Omega_{p}^{2 k-2}} \cdot L_{p}(K)\left(\Psi_{g}(k)\right) .
\end{aligned}
$$

Invoking Lemma 3.7 and (53), one finds that the powers of $\pi$ and the factorials (which, as functions of $k$, are not admissible) cancel out in the left-hand and right-hand sides of the formula in Lemma 3.8, which then follows.

For all integers $k \in \mathcal{U}_{g}^{\circ}, k>1$, define

$$
\omega(k)=I\left(\breve{g}_{k}^{*}, \breve{f}, \breve{h}^{*}\right) / I\left(\breve{g}_{k}, \breve{f}, \breve{h}\right)
$$

as in (25), and

$$
\mathfrak{f}(k)=\frac{\mathfrak{f}_{1}(k)}{\omega(k)}\left(\frac{\mathfrak{f}\left(\Psi_{g}(k)\right)}{\mathfrak{f}_{2}(k) \mathfrak{f}_{3}(k)}\right)^{2} .
$$

Let $\mathfrak{g}(\chi) \in \overline{\mathbb{Q}} \subset \mathbb{C}_{p}$ denote the Gauss sum associated to the character $\chi$, and define the number field $L_{0}=K\left(\sqrt{N_{g}}, \sqrt{N_{h}}, \mathfrak{g}(\chi), \psi_{g}, \psi_{h}\right)$. Theorem 3.3 will follow from the next result. 
THEOREM 3.9. The function $\mathfrak{f}(k)$ is $L_{0}$-admissible, and the following factorization of $p$-adic L-series is valid:

$$
\begin{aligned}
& \mathscr{L}_{p}^{g}(\breve{\mathbf{g}}, \breve{f}, \breve{h})(k)^{2} \cdot L_{p}(K)\left(\Psi_{g}(k)\right)^{2} \\
& \quad=\mathfrak{f}(k) \cdot L_{p}(f, K)\left(\Psi_{g h}(k)\right) \cdot L_{p}(f, K)\left(\Psi_{g h^{\prime}}(k)\right) .
\end{aligned}
$$

Moreover, there exist explicit elements $\lambda_{1}, \lambda_{2}, \lambda_{3} \in \mathbb{Q}\left(\psi_{g}, \psi_{h}\right)^{\times}$such that

$$
\begin{aligned}
L_{p}(f, K)\left(\Psi_{g h^{\prime}}(1)\right) & =\lambda_{1}^{2} \cdot \log _{E, p}\left(P_{1}\right)^{2}, \\
L_{p}(f, K)\left(\Psi_{g h}(1)\right) & =\lambda_{2}^{2} \cdot \log _{E, p}\left(P_{2}\right)^{2}, \\
L_{p}(K)\left(\Psi_{g}(1)\right) & =\lambda_{3} \cdot \log _{p}\left(u_{g_{\alpha}}\right) .
\end{aligned}
$$

Proof. We first prove (55): note that $\Psi_{g}(1)=\left(\psi_{g}^{\prime}\right)^{-2} \cdot \chi^{-1} \cdot \mathbf{N}_{K}=\left(\psi_{g} / \psi_{g}^{\prime}\right) \mathbf{N}_{K}$. By the functional equation of Katz's $p$-adic $L$-function quoted above, since $\psi_{g} / \psi_{g}^{\prime}$ is anticyclotomic, we have $L_{p}(K)\left(\left(\psi_{g} / \psi_{g}^{\prime}\right) \mathbf{N}_{K}\right)=L_{p}(K)\left(\psi_{g} / \psi_{g}^{\prime}\right)$, and hence (47) applies, yielding $L_{p}(K)\left(\Psi_{g}(k)\right)(1)=\lambda_{3}^{2} \log _{p}\left(u_{g_{\alpha}}\right)$ for some explicit value of $\lambda_{3} \in \mathbb{Q}\left(\psi_{g}, \psi_{h}\right)^{\times}$. Moreover,

$$
\begin{gathered}
c \quad \Psi_{g h}(1)=\left(\psi_{g}^{\prime}\right)^{-1} \cdot \psi_{h}^{-1} \cdot \mathbf{N}_{K}=\psi_{2} \cdot \mathbf{N}_{K}, \\
\Psi_{g h^{\prime}}(1)=\left(\psi_{g}^{\prime}\right)^{-1} \cdot\left(\psi_{h}^{\prime}\right)^{-1} \cdot \mathbf{N}_{K}=\psi_{1} \cdot \mathbf{N}_{K},
\end{gathered}
$$

and hence (49) can be applied, thereby proving (55).

In order to prove the admissibility of $\mathfrak{f}$ and the factorization formula, recall that we already saw in Lemma 3.6 that $\mathfrak{f}_{1}$ is $K\left(\psi_{g}, \psi_{h}\right)$-admissible, and likewise for $\mathfrak{f}_{2}$ and $\mathfrak{f}_{3}$. An inspection of the fudge factor $\mathfrak{f}\left(\Psi_{g}(k)\right)$ shows that it also is. Hence it remains to prove that $\omega(k)$ is $L_{0}$-admissible. Let us first show that $\omega$ interpolates to a meromorphic Iwasawa function on $\mathcal{U}_{g}$ such that (54) holds and $\omega(1) \in \mathbb{C}_{p}^{\times}$is a nonzero $p$-adic number, leaving for later that $\omega(1)$ actually lies in $L_{0}^{\times}$.

Note that Lemmas 3.6 and 3.8 give rise to the following identity for all integers $k \geqslant 0$ :

$$
\begin{aligned}
& \mathscr{L}_{p}^{g}(\breve{\mathbf{g}}, \breve{f}, \breve{h})(k) \cdot \mathscr{L}_{p}^{g}\left(\breve{\mathbf{g}}^{*}, \breve{f}, \breve{h}^{*}\right)(k) \cdot L_{p}(K)\left(\Psi_{g}(k)\right)^{2} \\
& \quad=\mathfrak{e}(k) \cdot \mathfrak{f}_{1}(k) \cdot\left(\frac{\mathfrak{f}\left(\Psi_{g}(k)\right)}{\mathfrak{f}_{2}(k) \mathfrak{f}_{3}(k)}\right)^{2} \cdot L_{p}(f, K)\left(\Psi_{g h}(k)\right) \cdot L_{p}(f, K)\left(\Psi_{g h^{\prime}}(k)\right),(56)
\end{aligned}
$$

where

$$
\mathfrak{e}(k):=\mathfrak{e}\left(\Psi_{g}(k)\right)^{2} \mathfrak{e}_{1}(k)=\left(\frac{\mathfrak{e}\left(\Psi_{g}(k)\right) \cdot \mathfrak{e}(k, 2,1)}{\mathfrak{e}\left(f, \Psi_{g h}(k)\right) \mathfrak{e}\left(f, \Psi_{g h^{\prime}}(k)\right)}\right)^{2} .
$$

An inspection of the factors appearing above shows that

$$
\mathfrak{e}(k)=1 \text { for all } k \geqslant 0 .
$$


More precisely, one checks that the four factors in the numerator $\mathcal{E}\left(g_{k}, f, h\right)$ of $\mathfrak{e}(k, 2,1)$ are equal to the four factors appearing in $\mathfrak{e}\left(f, \Psi_{g h}(k)\right) \mathfrak{e}\left(f, \Psi_{g h^{\prime}}(k)\right)$, and hence cancel out. In addition, the two factors showing up in $\mathfrak{e}\left(\Psi_{g}(k)\right)$ are identical to those in the denominator of $\mathfrak{e}(k, 2,1)$, and thus also cancel out.

Since the unit $u_{g_{\alpha}}$ and the points $P_{1}$ and $P_{2}$ are nontorsion, it also follows from (55) and (56) that neither $\mathscr{L}_{p}^{g}(\breve{\mathbf{g}}, \breve{f}, \breve{h})(k)$ nor $\mathscr{L}_{p}^{g}\left(\breve{\mathbf{g}}^{*}, \breve{f}, \breve{h}^{*}\right)(k)$ vanishes at $k=1$. Note that (32) implies that

$$
\omega(k)=\mathscr{L}_{p}^{g}\left(\breve{\mathbf{g}}^{*}, \breve{f}, \breve{h}^{*}\right)(k) / \mathscr{L}_{p}^{g}(\breve{\mathbf{g}}, \breve{f}, \breve{h})(k),
$$

from which it follows that $\omega \in \mathcal{L}_{g}$, with no poles at $k=1$ and satisfying $\omega(1) \neq 0$. The factorization formula claimed in the theorem is also a consequence of (56)-(58).

To finish the proof, we need to show that $\omega(1) \in L_{0}$. To do that, let $W_{N}$ denote the Atkin-Lehner operator induced by the matrix $\left(\begin{array}{cc}0 & -1 \\ N & 0\end{array}\right)$. As shown in [AL78] and [Hi2, Chapter X], it commutes with the Shimura-Maass operator, and its adjoint with respect to the Petersson scalar product is $-W_{N}$. This implies that

$$
I\left(\breve{g}_{k}, \breve{f}, \breve{h}\right)=I\left(W_{N}\left(\breve{g}_{k}\right), W_{N}(\breve{f}), W_{N}(\breve{h})\right) .
$$

By the formulae in [AL78], $W_{N}(\breve{h}(q))$ is a linear combination with coefficients in $\mathbb{Q}(h)$ of the modular forms $\lambda_{h} \cdot h^{*}\left(q^{d}\right)$, where $d$ ranges over the positive divisors of $N / N_{h}$ and $\lambda_{h}$ is the pseudoeigenvalue of the newform $h$ of level $N_{h}$ in the sense of [AL78], characterized by the equation $W_{N_{h}}(h)=\lambda_{h} h^{*}$. The analogous claim, with similar notation, holds for $g_{k}$ and $f$. It thus follows from for example [DR1, Lemma 2.12] that the ratio

$$
\omega(k)=I\left(\breve{g}_{k}^{*}, \breve{f}, \breve{h}^{*}\right) / I\left(W_{N}\left(\breve{g}_{k}\right), W_{N}(\breve{f}), W_{N}(\breve{h})\right)
$$

lies in $\mathbb{Q}\left(g_{k}, f, h, \lambda_{f}, \lambda_{g}, \lambda_{h}\right)$.

By [AL78, Theorem 2.1], since $a_{N_{g}}\left(g_{k}\right), a_{N_{f}}(f)$ and $a_{N_{h}}(h)$ are all nonzero, the pseudoeigenvalues of $f, g_{k}$, and $h$ are

$$
\lambda_{f}=-a_{N_{f}}(f), \quad \lambda_{g}=N_{g}^{(k-2) / 2} \mathfrak{g}\left(\chi^{-1}\right) / a_{N_{g}}\left(g_{k}\right), \quad \lambda_{h}=\mathfrak{g}(\chi) /\left(\sqrt{N_{h}} \cdot a_{N_{h}}(h)\right) .
$$

This implies that $\omega(1)$ lies in $L_{0}$, as claimed. Note that, since $p \nmid N$ and the coefficients $a_{N_{g}}\left(g_{k}\right)$ are Iwasawa functions on $\mathcal{U}_{g}$, the above formulae give rise to a rather explicit expression of $\omega(k)$ as a $p$-adic continuous function. Theorem 3.9 follows.

To conclude the proof of Theorem 3.3, recall that Proposition 2.6 recasts $\mathscr{L}_{p}^{g}(\breve{\mathbf{g}}, \breve{f}, \breve{h})(1)$ as the $p$-adic iterated integral. Combined with Theorem 3.9, we 
deduce that

$$
\left(\int_{\breve{\gamma}_{\alpha}} \breve{f} \cdot \breve{h}\right)^{2} \cdot \log _{p}\left(u_{g_{\alpha}}\right)^{2}=\mathfrak{f}(1) \log _{E, p}\left(P_{1}\right)^{2} \log _{E, p}\left(P_{2}\right)^{2}
$$

up to squares in $\mathbb{Q}\left(\psi_{g}, \psi_{h}\right)^{\times}$. Set $L=L_{0}(\sqrt{\mathfrak{f}(1)})$. Taking square roots in (59) shows that

$$
\int_{\breve{\gamma_{\alpha}}} \breve{f} \cdot \breve{h}=\lambda \cdot \frac{\log _{E, p}\left(P_{1}\right) \log _{E, p}\left(P_{2}\right)}{\log _{p}\left(u_{g_{\alpha}}\right)}
$$

for some $\lambda \in L^{\times}$, thereby proving Theorem 3.3.

3.3. Numerical evidence for inert primes. The proof of Conjecture ES based on Katz-style $p$-adic $L$-series and their factorizations breaks down when the prime $p$ is inert in $K$. Yet the units and points that arise can still be expressed in terms of elliptic units and Heegner points, at least in the rank $(1,1)$ setting, making this a natural first testing ground for Conjecture ES.

3.3.1. General remarks. Retain all the notation and assumptions of Section 3.2, but assume now that the prime $p$ is inert in $K$.

Let $K_{p}$ denote the completion of $K$ at $p$, a quadratic unramified extension of $\mathbb{Q}_{p}$. Since the Frobenius element $\sigma_{p}$ is a reflection in the dihedral group $\operatorname{Gal}(H / \mathbb{Q})$, the prime $p$ splits completely in $H / K$, and the choice of $\sigma_{p}$ amounts to choosing an embedding $H \hookrightarrow K_{p}$, which shall be fixed from now on.

Because $\varrho_{g}$ and $\varrho_{h}$ have dihedral projective image, it follows that $\sigma_{p}$ acts on $V_{g}$ (respectively, on $V_{h}$ ) with eigenvalues $\lambda$ and $-\lambda$ (respectively, $\lambda^{-1}$ and $-\lambda^{-1}$ ), for a suitable root of unity $\lambda$. Label the four values

$$
\alpha_{g}=\lambda, \quad \beta_{g}=-\lambda, \quad \alpha_{h}=\lambda^{-1}, \quad \beta_{h}=-\lambda^{-1},
$$

so that

$$
\alpha_{g} \alpha_{h}=1, \quad \alpha_{g} \beta_{h}=-1,
$$

as shall be assumed from now on. The decomposition

$$
V_{g h} \simeq V_{\psi_{1}} \oplus V_{\psi_{2}}
$$

of $\mathrm{Gal}(H / \mathbb{Q})$-modules continues to hold, but now $\sigma_{p}$ acts on both $V_{\psi_{1}}$ and $V_{\psi_{2}}$ as reflections, with eigenvalues 1 and -1 .

The asymmetry in the roles of $g$ and $h$ in the definition of the $p$-adic iterated integral continues to determine a pair $\left(\psi_{1}, \psi_{2}\right)$ of global characters of $G_{K}$ by requiring that $\psi_{1}$ and $\psi_{2}$ 'each be divisible by $\psi_{g}$ '. This pair is uniquely defined 
up to the action of $\mathrm{Gal}(K / \mathbb{Q})$, since replacing $\psi_{g}$ by $\psi_{g}^{\prime}$ has the effect of replacing $\left(\psi_{1}, \psi_{2}\right)$ by $\left(\psi_{2}^{\prime}, \psi_{1}^{\prime}\right)$. Fix $v_{\psi_{j}} \in V_{\psi_{j}}$ to be a nonzero vector in the one-dimensional $\psi_{j}$-eigenspace for the action of $G_{K}$ on $V_{\psi_{j}}$. With this choice in hand, set $v_{\psi_{j}^{\prime}}:=\sigma_{p} v_{\psi_{j}}$, as in the previous section. Given any $P \in \operatorname{Hom}\left(V_{\psi_{j}}\right.$, $\left.E(H)_{L}\right)$, we shall write

$$
P_{\psi_{j}}:=P\left(v_{\psi_{j}}\right), \quad P_{\psi_{j}^{\prime}}:=P\left(v_{\psi_{j}^{\prime}}\right) .
$$

LEMmA 3.10. The regulator $\operatorname{det}\left(R_{g_{\alpha}}\left(E, \varrho_{g h}\right)\right)$ is given by the following.

(1) If $r\left(E, V_{\psi_{1}}\right)=r\left(E, V_{\psi_{2}}\right)=1$,

$\operatorname{det} R_{g_{\alpha}}\left(E, \varrho_{g h}\right)=\log _{E, p}\left(P_{\psi_{1}}\right) \cdot \log _{E, p}\left(Q_{\psi_{2}}\right)-\log _{E, p}\left(P_{\psi_{1}^{\prime}}\right) \cdot \log _{E, p}\left(Q_{\psi_{2}^{\prime}}\right)$.

In particular, if $\psi_{1}$ (say) is a quadratic (genus) character, so that the point $P_{\psi_{1}}$ is a $\sigma_{p}$-eigenvector with eigenvalue $\iota= \pm 1$, then

$$
\operatorname{det} R_{g_{\alpha}}\left(E, \varrho_{g h}\right)=\log _{E, p}\left(P_{\psi_{1}}\right) \cdot \log _{E, p}\left(Q_{\psi_{2}}-\iota Q_{\psi_{2}^{\prime}}\right) .
$$

(2) If $r\left(E, V_{\psi_{1}}\right)=2$ and $r\left(E, V_{\psi_{2}}\right)=0$,

$\operatorname{det} R_{g_{\alpha}}\left(E, \varrho_{g h}\right)=\log _{E, p}\left(P_{\psi_{1}}\right) \cdot \log _{E, p}\left(Q_{\psi_{1}^{\prime}}\right)-\log _{E, p}\left(P_{\psi_{1}^{\prime}}\right) \cdot \log _{E, p}\left(Q_{\psi_{1}}\right)$,

where $(P, Q)$ is a basis for $\operatorname{Hom}_{G_{\mathbb{Q}}}\left(V_{\psi_{1}}, E(H)_{L}\right)$.

Observe in particular that the regulator vanishes in the special case where there are no global points $\left(P_{1}, P_{2}\right) \in E(H)_{L}^{\varrho_{1}} \times E(H)_{L}^{\varrho_{2}}$ belonging to distinct eigenspaces for the action of $\sigma_{p}$; this exceptional scenario in which the regulator is degenerate occurs precisely when

$$
\left.\operatorname{dim}\left(\varrho_{1}\right)=\operatorname{dim}\left(\varrho_{2}\right)=1 \text { (so that } \psi_{1}^{2}=\psi_{2}^{2}=1\right) \text { and } \varrho_{1}(p)=\varrho_{2}(p) .
$$

If (60) holds, Conjecture ES predicts that the iterated integrals attached to $(f, g, h)$ vanish, in spite of the running hypothesis $r_{\text {an }}\left(E, \varrho_{1}\right)=r_{\text {an }}\left(E, \varrho_{2}\right)=1$. See Example 4.3 below for a numerical verification of this interesting phenomenon.

Suppose for the rest of this section that (60) is not satisfied, so that, switching $\varrho_{1}$ and $\varrho_{2}$ if necessary, it may be assumed that 1 and -1 are eigenvalues of $\varrho_{1}\left(\sigma_{p}\right)$ and $\varrho_{2}\left(\sigma_{p}\right)$, respectively. Recall furthermore the points $P_{\psi_{i}}^{ \pm}$defined from Heegner points in Lemma 3.1 and (43), and the unit $u_{\psi_{0}}^{-}$defined in (44) from elliptic units. Assuming (38) or (40), Conjecture ES predicts that there is some 
choice of test vectors $\breve{f}, \breve{\gamma}$, and $\breve{h}$ for which

$$
\int_{\breve{\gamma}} \breve{f} \cdot \breve{h}= \begin{cases}\frac{\log _{E, p}\left(P_{\psi_{1}}^{+}\right) \log _{E, p}\left(P_{\psi_{2}}^{-}\right)-\log _{E, p}\left(P_{\psi_{1}}^{-}\right) \log _{E, p}\left(P_{\psi_{2}}^{+}\right)}{\log _{p}\left(u_{\psi_{0}}^{-}\right)} & \text {if } \psi_{1}^{2}, \psi_{2}^{2} \neq 1, \\ \frac{\log _{E, p}\left(P_{\psi_{1}}^{+}\right) \log _{E, p}\left(P_{\psi_{2}}^{-}\right)}{\log _{p}\left(u_{\psi_{0}}^{-}\right)} & \text {and } V_{\psi_{1}} \neq V_{\psi_{2}},\end{cases}
$$

3.3.2. Numerical evidence. The following experiment in support of (61) gave the authors their first evidence that the $p$-adic iterated integrals continue to have geometric meaning beyond the setting treated in Section 3.2.

EXAMPle 3.11. Consider the elliptic curve

$$
E: y^{2}+x y+y=x^{3}+x^{2}+x
$$

of conductor 83 labelled $83 a$ in Cremona's tables, and write $f$ for the associated weight-two newform. Let $\chi$ be the odd quadratic character of conductor 83 . The space $S_{1}(83, \chi)$ has dimension one, and is spanned by the theta series $g:=\theta_{\psi_{g}}$ attached to a cubic character $\psi_{g}$ of $G=\operatorname{Gal}(H / K)$, where $H$ is the Hilbert class field of $K=\mathbb{Q}(\sqrt{-83})$, which has class number 3. The representation $\rho_{g}$ is equal to the induced representation $V_{\psi_{g}}$. Let $h=E(1, \chi) \in M_{1}(83, \chi)$ be the Eisenstein series attached to the pair $(1, \chi)$, whose Galois representation $\varrho_{h}$ is induced from the trivial character $\psi_{h}=1$ of $G$. Since $g$ and $h$ have rational Fourier coefficients, it will be enough to take $L=\mathbb{Q}$ (although the representations are in fact defined over $\mathbb{Q}\left(\zeta_{3}\right)$ ). The representations $V_{g g}$ and $V_{g h}$ decompose into irreducible constituents as follows:

$$
\begin{gathered}
V_{g g}=V_{\psi_{g}^{2}} \oplus V_{\psi_{g} \cdot \psi_{g}^{-1}}=V_{\psi_{g}} \oplus \mathbb{Q} \oplus \mathbb{Q}(\chi)=: \varrho_{1} \oplus \varrho_{2} \oplus \varrho_{3}, \\
V_{g h}=V_{\psi_{g} \cdot \psi_{h}} \oplus V_{\psi_{g} \cdot \psi_{h}^{-1}}=V_{\psi_{g}} \oplus V_{\psi_{g}}=\varrho_{1} \oplus \varrho_{1} .
\end{gathered}
$$

A direct calculation shows that

$$
r\left(E, \varrho_{1}\right)=r\left(E, \varrho_{2}\right)=1, \quad r\left(E, \varrho_{3}\right)=0,
$$

and similarly for the analytic ranks. More precisely, the Mordell-Weil group

$$
E(H)_{\mathbb{Q}}=E(H)_{\mathbb{Q}}^{V_{\psi g}} \oplus E(\mathbb{Q})_{\mathbb{Q}}
$$

is of rank 3, and is generated by the Heegner point

$$
P=\left(t,-t^{2}-2\right) \in E(H) \quad \text { where } t^{3}-t^{2}+t-2=0,
$$


along with its Galois translates $P^{\sigma}$ and $P^{\sigma^{2}}$, where $\sigma$ is a generator of $\operatorname{Gal}(H / K)$. The vector spaces $E(\mathbb{Q})_{\mathbb{Q}}$ and $E(H)_{\mathbb{Q}}^{V_{\psi_{g}}}$ are generated by

$$
P_{\mathbb{Q}}:=P+P^{\sigma}+P^{\sigma^{2}}=(1,-3), \quad \text { and } \quad\left\{\begin{array}{l}
P_{\psi_{g}}^{+}:=2 P-P^{\sigma}-P^{\sigma^{2}}, \\
P_{\psi_{g}}^{-}:=P^{\sigma}-P^{\sigma^{2}} .
\end{array}\right.
$$

Let $p=5$, which is in inert in $K$. The cubic polynomial in (62) has exactly one root over $\mathbb{Q}_{5}$. There is therefore a unique embedding of $H$ into $\mathbb{C}_{5}$ for which the Heegner point $P$ maps to $E\left(\mathbb{Q}_{5}\right)$; the images of the points $P^{\sigma}$ and $P^{\sigma^{2}}$ are then interchanged by the Frobenius element $\sigma_{5}$.

The Stark unit that enters into the denominator of the conjectural expression for the $p$-adic iterated integral is the elliptic unit defined by $u_{\psi_{0}}^{-}=u^{\sigma} / u^{\sigma^{2}}$, so that

$$
\log _{p}\left(u_{\psi_{0}}^{-}\right)=\log _{p}\left(u^{\sigma}\right)-\log _{p}\left(u^{\sigma^{2}}\right),
$$

where $u \in H$ is the unique root of the polynomial

$$
x^{3}-2 x^{2}-2 x-1
$$

whose image under the previously chosen embedding of $H$ belongs to $\mathbb{Q}_{5}$.

Let $\gamma_{ \pm 1} \in S_{1}^{\text {oc,ord }}(83, \chi)^{\vee}$ be the dual basis to $g_{ \pm 1}=g(q) \mp g\left(q^{5}\right)$ in the space of 5-adic overconvergent modular forms, which maps $g_{ \pm 1}$ to 1 and all other generalized eigenspaces in $S_{1}^{\text {oc,ord }}(83, \chi)$ to 0 . A numerical calculation of 5 -adic iterated integrals shows to 70 digits of 5-adic precision

$$
\begin{aligned}
& \int_{\gamma_{ \pm 1}} f \cdot g=-1688341751390720654446425615337716320464561776401, \\
& \int_{\gamma_{ \pm 1}} f \cdot h=-2981481189608571355501040629889815717824676581453 .
\end{aligned}
$$

Let $\mathcal{E}\left(g_{ \pm 1}, f, g\right)$ denote the algebraic factor defined at the start of Section 5.2. In all the examples where $p$ is inert, this factor is always equal to

$$
\mathcal{E}\left(g_{ \pm 1}, f, g\right):=\left(1-\frac{a_{p}(f)}{p}+\frac{1}{p}\right)\left(1+\frac{a_{p}(f)}{p}+\frac{1}{p}\right) \in \mathbb{Q} .
$$

The following identities were checked to 70 digits of 5-adic precision:

$$
\begin{aligned}
\int_{\gamma_{ \pm 1}} f \cdot g & =\frac{5 \cdot \mathcal{E}\left(g_{ \pm 1}, f, g\right) \cdot \log _{E, 5}\left(P_{\mathbb{Q}}\right) \log _{E, 5}\left(P_{\psi_{g}}^{-}\right)}{24 \cdot \log _{5}\left(u_{\psi_{0}}^{-}\right)} \\
\int_{\gamma_{ \pm 1}} f \cdot h & =-\frac{5 \cdot \mathcal{E}\left(g_{ \pm 1}, f, h\right) \cdot \log _{E, 5}\left(P_{\psi_{g}}^{+}\right) \log _{E, 5}\left(P_{\psi_{g}}^{-}\right)}{24 \cdot \log _{5}\left(u_{\psi_{0}}^{-}\right)}
\end{aligned}
$$


in perfect agreement with the prediction (61) for both $\gamma_{+1}$ and $\gamma_{-1}$. Note that $u_{\psi_{0}}^{-}$ is a unit in the (-1)-eigenspace for $\sigma_{5}$ while the numerators involve points in both eigenspaces, so that the resulting ratio belongs to $\mathbb{Q}_{5}$.

Although the Heegner point $P$ is only defined up to sign, it is worth noting that the right-hand sides of (63) and (64) are unchanged if $P$ is replaced by $-P$.

Formulae similar to (63) and (64) (with the terms 5 and 24 in the numerator and denominator replaced by $p$ and $4(p+1)$, respectively) were verified to 15 digits of 13 -adic precision (respectively, 10 digits of 19 -adic precision) replacing $p=5$ by the inert primes $p=13$ and $p=19$. Similar experiments were also performed with the class number 3 field $K=\mathbb{Q}(\sqrt{-331})$ and the inert prime $p=7$, where the same formulae (mutatis mutandis) were verified to 10 significant 7 -adic digits.

The next example also involves Heegner points, but is one of the most illuminating in this paper, insofar as it concerns a rank- $(1,1)$ setting of Conjecture ES in which nonetheless the regulator $R_{g_{\alpha}}\left(E, \varrho_{g h}\right)$ is not factorizable. It illustrates a feature of Conjecture ES which initially surprised the authors, namely, that the numerator on the right-hand side of Conjecture ES need not, in contrast with what one might expect from Theorem 3.3, be the product of logarithms of two points, but may involve a linear combination of such products.

EXAMPLE 3.12. Consider the curve

$$
E: y^{2}+x y+y=x^{3}+x^{2}-2 x
$$

of conductor 79 labelled $79 a$ in Cremona's tables, and write $f$ for the associated weight-two newform.

Let $\chi$ be the odd Dirichlet character of order 2 and conductor 79 associated to the imaginary quadratic field $K=\mathbb{Q}(\sqrt{-79})$, of class number 5. As in the previous example, denote by $H$ the Hilbert class field of $K$, and set $G=$ $\operatorname{Gal}(H / K)$. The space $S_{1}(79, \chi)$ has dimension two, and is spanned by a pair of dihedral eigenforms with coefficients in the field $L=\mathbb{Q}(\sqrt{5})$ generated by the golden ratio $\omega=(1+\sqrt{5}) / 2$, namely

$$
g=q+(-1+\omega) q^{2}+(1-\omega) q^{4}-\omega q^{5}-q^{8}+q^{9}-q^{10}+\cdots
$$

and its Galois conjugate $g^{\dagger}$. Let $h=E(1, \chi) \in M_{1}(79, \chi)$ be the Eisenstein series attached to the pair $(1, \chi)$. After fixing a quintic character $\psi$ of $G$, it can be assumed that

$$
V_{g}=V_{\psi^{2}}=V_{\psi^{3}}, \quad V_{g^{\dagger}}=V_{\psi}=V_{\psi^{4}}, \quad V_{h}=V_{\psi_{h}}=L \oplus L\left(\chi_{K}\right),
$$


where $\psi_{h}$ is the trivial character of $G_{K}$. The representations $V_{g^{\dagger} g}$ and $V_{g^{\dagger} h}$ decompose into irreducible summands as follows:

$$
\begin{gathered}
V_{g^{\dagger} g}=V_{\psi \cdot \psi^{-2}} \oplus V_{\psi \cdot \psi^{2}}=V_{\psi} \oplus V_{\psi^{2}}=: \varrho_{1} \oplus \varrho_{2}, \\
V_{g^{\dagger} h}=V_{\psi \cdot \psi_{h}} \oplus V_{\psi \cdot \psi_{h}^{-1}}=V_{\psi} \oplus V_{\psi}=\varrho_{1} \oplus \varrho_{1} .
\end{gathered}
$$

A Heegner point calculation reveals that the Mordell-Weil group $E(H)$ has rank 5 , and is generated by the Heegner point

$$
P=\left(t, \frac{-t^{4}-3 t^{3}+2 t^{2}+7 t-6}{3}\right) \quad \text { where } t^{5}+t^{4}-5 t^{3}-3 t^{2}+8 t-3=0,
$$

and its Galois translates $P^{\sigma}, P^{\sigma^{2}}, P^{\sigma^{3}}$, and $P^{\sigma^{4}}$, where $\sigma$ is a generator of Gal $(H / K)$, chosen in such a way that $\psi(\sigma)=\zeta_{5}$ with $\zeta_{5}$ a primitive fifth root of unity satisfying $\zeta_{5}+\zeta_{5}^{4}=-\omega$. Let $\omega^{\prime}=(1-\sqrt{5}) / 2$ denote the conjugate of the golden ratio. The $L$-vector space $E(H)_{L}^{\varrho_{1}}$ is then spanned by the points

$$
\begin{aligned}
& P_{1}^{+}=2 P-\omega P^{\sigma}-\omega^{\prime} P^{\sigma^{2}}-\omega^{\prime} P^{\sigma^{3}}-\omega P^{\sigma^{4}}, \\
& P_{1}^{-}=\left(\zeta_{5}-\zeta_{5}^{4}\right)\left(P^{\sigma}-\omega P^{\sigma^{2}}+\omega P^{\sigma^{3}}-P^{\sigma^{4}}\right),
\end{aligned}
$$

and $E(H)_{L}^{\varrho_{2}}$ has a similar basis $\left(P_{2}^{+}, P_{2}^{-}\right)$obtained by replacing $\zeta_{5}$ by $\zeta_{5}^{3}$, and thus $\omega$ by its conjugate $\omega^{\prime}$, in the above expressions for $P_{1}^{+}$and $P_{1}^{-}$.

Let $p=29$ be the smallest prime which is inert in $K$ but split in $L / \mathbb{Q}$. (The latter property is not essential to the conjectures, but makes it easier to calculate the $p$-adic iterated integrals.) Fix the unique embedding of $H$ into $\mathbb{C}_{p}$ for which the image of $P$ belongs to $E\left(\mathbb{Q}_{p}\right)$, and for which $\sigma_{p}$ interchanges $P^{\sigma}$ with $P^{\sigma^{4}}$ and $P^{\sigma^{2}}$ with $P^{\sigma^{3}}$.

For the elliptic unit, one may start with $u$ as the unique root of $x^{5}-1014 x^{4}+$ $299243 x^{3}-21035 x^{2}+195 x-1$ in $H \cap \mathbb{Q}_{29}$, and define $u_{g_{ \pm 1}^{\dagger}}$ in exactly the manner as $P_{2}^{-}$was defined above (only replacing $P$ by $u$ and using additive notation for $\left.\left(\mathcal{O}_{H}^{\times}\right)_{L}\right)$; that is,

$$
u_{g_{ \pm 1}^{\dagger}}:=\left(\zeta_{5}^{3}-\zeta_{5}^{2}\right)\left(u^{\sigma}-\omega u^{\sigma^{2}}+\omega u^{\sigma^{3}}-u^{\sigma^{4}}\right) .
$$

Note that $u_{g_{+1}^{\dagger}}$ may be alternatively denoted $u_{\psi_{0}}^{-}$.

Let $\gamma_{ \pm 1}^{\dagger} \in S_{1}^{\text {oc,ord }}(79 \cdot 23, \chi)^{\vee}$ be the dual element to $g_{ \pm 1}^{\dagger}$, defined as before. A numerical calculation of $p$-adic iterated integrals reveals that

$$
\begin{aligned}
& \int_{\gamma_{ \pm 1}^{\dagger}} f \cdot h=371806430046362 \bmod 29^{10} \\
& \int_{\gamma_{ \pm 1}^{\ddagger}} f \cdot g=259114157339642 \bmod 29^{10} .
\end{aligned}
$$


The following identity was then verified to ten digits of 29-adic precision:

$$
\begin{aligned}
& \int_{\gamma_{ \pm 1}^{\dagger}} f \cdot g \\
& =\frac{p \cdot \mathcal{E}\left(g_{ \pm 1}^{\dagger}, f, g\right) \cdot\left(\log _{E, 29}\left(P_{1}^{+}\right) \log _{E, 29}\left(P_{2}^{-}\right)-\log _{E, 29}\left(P_{1}^{-}\right) \log _{E, 29}\left(P_{2}^{+}\right)\right)}{4 \cdot 5 \cdot \sqrt{5} \log _{29}\left(u_{g_{ \pm 1}^{\dagger}}\right)},
\end{aligned}
$$

where here $\sqrt{5}$ denotes the unique 29-adic square root of 5 which is congruent to 18 modulo 29 .

Turning to $\int_{\gamma_{ \pm 1}^{\dagger}} f \cdot h$, notice that $\mathcal{E}\left(g_{ \pm 1}^{\dagger}, f, h\right)=\mathcal{E}\left(g_{ \pm 1}^{\dagger}, f, g\right)(=864 / 841)$ and the transcendental factor $\log _{29}\left(u_{g^{\dagger}}\right)$ expected on the right-hand side of the formula is the same as for $\int_{\gamma_{ \pm 1}^{\dagger}} f \cdot g$. Thus these factors should cancel upon taking a ratio, and indeed one observes (modulo $29^{10}$ ) the identity

$$
\begin{aligned}
\frac{\int_{\gamma_{ \pm 1}^{\dagger}} f \cdot g}{\int_{\gamma_{ \pm 1}^{\dagger}} f \cdot h} & =\frac{1}{2} \cdot \frac{\log _{E, 29}\left(P_{1}^{+}\right) \log _{E, 29}\left(P_{2}^{-}\right)-\log _{E, 29}\left(P_{1}^{-}\right) \log _{E, 29}\left(P_{2}^{+}\right)}{\log _{E, 29}\left(P_{1}^{+}\right) \log _{E, 29}\left(P_{1}^{-}\right)} \\
& =\frac{1}{2}\left(\frac{\log _{E, 29}\left(P_{2}^{-}\right)}{\log _{E, 29}\left(P_{1}^{-}\right)}-\frac{\log _{E, 29}\left(P_{2}^{+}\right)}{\log _{E, 29}\left(P_{1}^{+}\right)}\right),
\end{aligned}
$$

which suggests that

$$
\int_{\gamma_{ \pm 1}^{\dagger}} f \cdot h=\frac{p \cdot \mathcal{E}\left(g_{ \pm 1}^{\dagger}, f, h\right) \cdot \log _{E, 29}\left(P_{1}^{+}\right) \log _{E, 29}\left(P_{1}^{-}\right)}{2 \cdot 5 \cdot \sqrt{5} \log _{29}\left(u_{g_{ \pm 1}^{\dagger}}\right)}
$$

Both equations (65) and (67) are in perfect agreement with Conjecture ES.

REMARK 3.13. It was a ratio identity like the one in (66) (for a simpler ratio in Example 3.11) which first convinced the authors that the $p$-adic iterated integral had geometric meaning in the setting where $p$ is inert, even prior to their full understanding of the logarithm of the Stark unit occurring in the denominator of the identity of Conjecture ES.

We now turn to our first numerical example in the rank-2 setting, illustrating a notable feature of Conjecture ES, namely that its right-hand side can be a nontrivial $p$-adic combination of logarithms of global points in a 'genuine rank2 ' setting involving a primitive $L$-function with a double zero at the centre.

EXAMPLE 3.14. We take the curve

$$
E: y^{2}+x y+y=x^{3}-7 x+5
$$


of conductor 57 labelled $57 b$ in Cremona's tables, and write $f$ for the attached newform of weight two.

Let $\chi$ be the odd Dirichlet character of order 2 and conductor 23 associated to the imaginary quadratic field $K=\mathbb{Q}(\sqrt{-23})$, of class number 3 . The space $S_{1}(23, \chi)$ has dimension one, and is spanned by the theta series $g:=\theta_{\psi_{g}}$ attached to a cubic character $\psi_{g}$ of $G=\operatorname{Gal}(H / K)$, where $H$ is the class field of $K$. As in Example 3.11, we find

$$
V_{g g}=V_{\psi_{g}} \oplus L \oplus L(\chi) \quad \text { with } L=\mathbb{Q}\left(\zeta_{3}\right),
$$

but a calculation reveals that here

$$
r\left(E, V_{\psi_{g}}\right)=2, \quad r(E, L)=r(E, L(\chi))=0,
$$

and likewise for the analytic ranks. Here, the Heegner hypothesis is not satisfied, but the descent code in MAGMA can be used to construct the required global points in $E(H)_{L}^{V_{\psi_{g}}}$. Over the cubic subfield

$$
\mathbb{Q}(a), \quad a^{3}-a^{2}+2 a-1=0
$$

of $H$, the curve $E$ has rank 2 with generators for the Mordell-Weil lattice

$$
P:=\left(9 a^{2}-4 a+17,-45 a^{2}+20 a-80\right), \quad Q:=\left(a^{2}+3,2 a^{2}+2\right) .
$$

We embed $\mathbb{Q}(a)$ into $H=\mathbb{Q}(b)$, where $b^{6}-6 b^{4}+9 b^{2}+23=0$, by taking $a=\left(b^{4}-2 b^{2}+1\right) / 9$. Taking $\sigma$ as a generator for $G$, we compute four points

$$
\begin{array}{ll}
P_{\psi_{g}}^{+}:=2 P-P^{\sigma}-P^{\sigma^{2}}, & P_{\psi_{g}}^{-}:=P^{\sigma}-P^{\sigma^{2}}, \\
Q_{\psi_{g}}^{+}:=2 Q-Q^{\sigma}-Q^{\sigma^{2}}, & Q_{\psi_{g}}^{-}:=Q^{\sigma}-Q^{\sigma^{2}},
\end{array}
$$

and similarly a unit $u_{\psi_{0}}^{-}$starting from the root $\left(b^{4}-5 b^{2}+4\right) / 9$ of $x^{3}-x-1$ in $H$. We take $p=19$, and find we may embed $H$ into $\mathbb{C}_{19}$ so that $P, Q$, and $u$ are defined over $\mathbb{Q}_{19}$.

To compute the relevant 19 -adic iterated integral we must work in 19 -adic level $3 \cdot 23$, and so choose a 3 -stabilization $\breve{g}$ of $g$. We find $x^{2}-a_{3}(g) x+$ $\chi(3)=\left(x-\zeta_{3}\right)\left(x-\zeta_{3}^{2}\right)$ and take $\breve{g}$ to be the 3-stabilization with $U_{3}$-eigenvalue $\zeta_{3}$. (With the other choice the formula is exactly the same, except the algebraic factor is replaced by its complex conjugate.) We embed $\mathbb{Q}\left(\zeta_{3}\right)$ into $\mathbb{Q}_{19}$ so that $\zeta_{3} \equiv 7 \bmod 19$, and find

$$
\int_{\breve{\gamma}_{ \pm 1}} f \cdot g=-25103076413984358720047537708218 \bmod 19^{25} .
$$


One checks to 25 digits of 19 -adic precision that

$$
\int_{\breve{\gamma}_{ \pm 1}} f \cdot g=\frac{63 \zeta_{3}+18}{26 \cdot 19} \cdot \frac{\log _{E, 19}\left(P_{\psi_{g}}^{+}\right) \log _{E, 19}\left(Q_{\psi_{g}}^{-}\right)-\log _{E, 19}\left(P_{\psi_{g}}^{-}\right) \log _{E, 19}\left(Q_{\psi_{g}}^{+}\right)}{\log _{p} u_{\psi_{0}}^{-}},
$$

as predicted by Conjecture ES.

In the next example, the relevant Heegner point arises from a Shimura curve rather than a classical modular curve parameterization. Note that Conjecture ES in principle allows the efficient computation of these Heegner points in terms of the Fourier expansions of classical modular forms rather than working with their Jacquet-Langlands lifts. For the purpose of verifying Conjecture ES, the authors did not actually calculate the Heegner points arising from the Shimura curve parameterization, which raises significant computational challenges, but rather found appropriate global points by general descent methods.

EXAMPle 3.15. Consider the curve

$$
E: y^{2}+y=x^{3}+x^{2}+9 x+1
$$

of conductor 35 labelled $35 a$ in Cremona's tables, and write $f$ for the associated weight-two newform. Let $K=\mathbb{Q}(\sqrt{-23})$ be the smallest imaginary quadratic field of class number 3, and note that both primes dividing 35 are inert in $K$. This is the simplest setting where there is no Heegner point on $E$ arising from a modular curve, and in which one must rather work with the Shimura curve attached to the indefinite quaternion algebra ramified at 5 and 7.

Let $\chi$ be the quadratic Dirichlet character of conductor 23. Then $S_{1}(23, \chi)$ is one dimensional, and is spanned by the single dihedral form

$$
g=q-q^{2}-q^{3}+q^{6}+q^{8}-q^{13}-q^{16}+\cdots .
$$

Let $H$ be the Hilbert class field of $K$, set $G=\operatorname{Gal}(H / K)$, and let $\varrho_{g}$ be the representation induced from a cubic character $\psi_{g}$ of $G$ with image contained in $L=\mathbb{Q}\left(\zeta_{3}\right)$.

The representation $V_{g g}$ decomposes as

$$
V_{g g}=V_{\psi_{g} \cdot \psi_{g}} \oplus V_{\psi_{g} \cdot \psi_{g}^{-1}}=V_{\psi_{g}} \oplus L(\chi) \oplus L
$$

A direct calculation reveals that

$$
r\left(E, V_{\psi_{g}}\right)=r(E, L(\chi))=1, \quad r(E, L)=\operatorname{rank}(E(\mathbb{Q}))=0,
$$

and the same is true of the analytic ranks. Let $P_{K}$ be a generator of $E(K)$ up to torsion, which lies in the -1 eigenspace for the action of the involution in 
$\operatorname{Gal}(K / \mathbb{Q})$, and let $P$ be a $\mathbb{Q}[\mathrm{Gal}(H / K)]$-module generator of $E(H) \otimes \mathbb{Q}$. The points $P_{K}$ and $P$ were not constructed using Lemma 3.1, since the Heegner point evoked in this lemma is difficult to compute directly in practice. Instead, one just takes

$$
\begin{gathered}
P_{K}:=\left(-5, \frac{-5 \sqrt{-23}-1}{2}\right), \\
P:=\left(\frac{-7 t^{4}+14 t^{2}+29}{36}, \frac{7 t^{4}-14 t^{2}-68}{24}\right) \in E(H),
\end{gathered}
$$

where $t$ is a normal basis generator for $H$ over $\mathbb{Q}$ satisfying the equation

$$
t^{6}-6 t^{4}+9 t^{2}+23=0 .
$$

The point $P$ just turned out to be the first point in a basis for the rank-3 MordellWeil group $E(H)$ computed by MAGMA.

Let $p=5$ be the smallest prime which is inert in $K$. After a suitable choice of embedding of $H$ into $K_{5}$ for which $P$ maps to $E\left(\mathbb{Q}_{5}\right)^{-}$, the Frobenius element $\sigma_{5}$ interchanges $P^{\sigma}$ and $P^{\sigma^{2}}$. With these conventions, we may set

$$
P_{\psi_{g}}^{+}:=2 P-\left(P^{\sigma}+P^{\sigma^{2}}\right) \text {. }
$$

Both $f$ and $g$ are 5-adic overconvergent modular forms of level 7 and 23, respectively. Stabilize $g$ to an eigenform $\breve{g}=g(q)-g\left(q^{7}\right)$ of level $161=7 \cdot 23$ with $U_{7}$ eigenvalue 1 . Then $\breve{g}$ has two stabilizations

$$
\breve{g}_{ \pm 1}=\breve{g}(q) \mp \breve{g}\left(q^{5}\right)
$$

at 5, which can be viewed either as classical modular forms of level 161.5 or as 5-adic overconvergent forms of level 161. Write $\breve{\gamma}_{ \pm 1} \in S_{1}^{\text {oc,ord }}(7 \cdot 23, \chi)^{\vee}$ for the usual dual element to $\breve{g}_{ \pm 1}$. A direct evaluation of the 5-adic iterated integral reveals that

$$
\int_{\breve{\gamma}+1} f \cdot g=-\int_{\breve{\gamma}-1} f \cdot g=376357248757242028241 \bmod 5^{30} .
$$

Starting with the elliptic unit $u$ which is the unique root of $x^{3}+6 x^{2}+29 x-1$ in $H \cap \mathbb{Q}_{5}$, the unit $u^{-}$is defined by $u^{-}=\left(u^{\tau} / u^{\tau^{2}}\right)$. The following identity was then checked to 30 digits of 5-adic precision:

$$
\int_{\breve{\gamma}_{+1}} f \cdot g=\frac{3 \cdot 7 \cdot \log _{E, 5}\left(P_{K}\right) \cdot \log _{E, 5}\left(P_{\psi_{g}}^{+}\right)}{2 \cdot 5 \cdot \log _{5}\left(u^{-}\right)},
$$

as predicted by (61). Note that once the point $P_{K}$ is known, this identity leads to an explicit analytic formula for a global point on $E$ over the Hilbert class 
field $H$, namely $P_{\psi_{g}}^{+}$, without passing through the calculation of the Shimura curve parameterization of $E$, as would be necessary if one were to resort to Heegner points instead of $p$-adic iterated integrals.

\section{Stark-Heegner points and theta series of real quadratic fields}

4.1 Theoretical preliminaries. This section focusses on the case where $g$ and $h$ are theta series attached to characters - denoted $\psi_{g}$ and $\psi_{h}$, respectivelyof mixed signature $(+,-)$ of the same real quadratic field $K$. The self-duality assumption on $V_{g h}$ implies that

$$
\psi_{1}:=\psi_{g} \psi_{h}, \quad \psi_{2}:=\psi_{g} \psi_{h}^{\prime}
$$

are dihedral characters of $K$, which are totally even and totally odd respectively, and that

$$
V_{g}=V_{\psi_{g}}, \quad V_{h}=V_{\psi_{h}}, \quad V_{g h}=V_{\psi_{1}} \oplus V_{\psi_{2}} .
$$

As in the previous section, the Hasse-Weil-Artin $L$-function factors as

$$
L\left(E, \varrho_{g h}, s\right)=L\left(E / K, \psi_{1}, s\right) \cdot L\left(E / K, \psi_{2}, s\right),
$$

and Hypothesis A is fulfilled as soon as the conductors of $\psi_{g}$ and $\psi_{h}$ are coprime to $N_{f}$. In all examples where Conjecture ES is tested below, Hypothesis B holds because $L\left(E / K, \psi_{1}, 1\right)=L\left(E / K, \psi_{2}, 1\right)=0$.

Let $p$ be a rational prime, at which the representations $V_{g}$ and $V_{h}$ are unramified, and let $\alpha_{g}$ and $\beta_{g}$ (respectively, $\alpha_{h}$ and $\beta_{h}$ ) denote as before the Frobenius eigenvalues for $V_{g}$ (respectively, $V_{h}$ ) attached to this prime. In order for $g$ to satisfy Hypotheses $C$ and $C^{\prime}$, it is necessary and sufficient to assume that

$$
p \text { is inert in } K \text {. }
$$

In that case, by the remarks surrounding (22), the Stark unit $u_{g_{\alpha}}=: u_{K}$ of Conjecture ES can be taken to be equal to the fundamental unit of $K$, and in particular it depends only on $K$ and not on $\psi_{g}$.

Assumption (68) also implies that the Frobenius element $\sigma_{p}$ corresponds to reflections in the dihedral groups cut out by the representations $V_{\psi_{1}}$ and $V_{\psi_{2}}$. As in Section 3.3, it is assumed that

$$
\alpha_{g} \alpha_{h}=1, \quad \alpha_{g} \beta_{h}=-1, \quad \beta_{g} \alpha_{h}=-1, \quad \beta_{g} \beta_{h}=1 .
$$

The eigenvalues of $\sigma_{p}$ on $V_{\psi_{1}}$ are $(1,-1)$, and likewise for $V_{\psi_{2}}$. Note in particular that, in this setting, the representation $V_{g h}$ is never regular at $p$, even though the representations $V_{g}, V_{h}, V_{\psi_{1}}$, and $V_{\psi_{2}}$ are all regular. 
Consider first the 'generic' setting where neither of the characters $\psi_{1}$ or $\psi_{2}$ is a quadratic (genus) character, so that the representations $V_{\psi_{1}}$ and $V_{\psi_{2}}$ are both irreducible. Conjecture ES then predicts the existence of points $P_{1} \in E(H)_{L}^{V_{\psi_{1}}}$ and $P_{2} \in E(H)_{L}^{V_{\psi_{2}}}$ whose projections onto the different $\sigma_{p}$-eigenspaces

$$
\begin{array}{ll}
P_{1}^{+} \in\left(E(H)_{L}^{V_{\psi_{1}}}\right)^{\sigma_{p}=+1}, & P_{2}^{-} \in\left(E(H)_{L}^{V_{\psi_{2}}}\right)^{\sigma_{p}=-1}, \\
P_{1}^{-} \in\left(E(H)_{L}^{V_{\psi_{1}}}\right)^{\sigma_{p}=-1}, & P_{2}^{+} \in\left(E(H)_{L}^{V_{\psi_{2}}}\right)^{\sigma_{p}=+1},
\end{array}
$$

are related to a suitable $p$-adic iterated integral by

$$
\int_{\breve{\gamma} \alpha} \breve{f} \cdot \breve{h} \stackrel{?}{=} \frac{\log _{E, p}\left(P_{1}^{+}\right) \log _{E, p}\left(P_{2}^{-}\right)-\log _{E, p}\left(P_{1}^{-}\right) \log _{E, p}\left(P_{2}^{+}\right)}{\log _{p}\left(u_{K}\right)} .
$$

This conjecture becomes slightly easier to verify when one of the characters $\psi_{1}$ or $\psi_{2}$ is a quadratic (genus) character. Assume without loss of generality that this is the case for $\psi_{1}$, so that

$$
V_{\psi_{1}}=L\left(\chi_{1}\right) \oplus L\left(\chi_{2}\right)
$$

becomes reducible as a $G_{\mathbb{Q}}$-module, breaking up into two one-dimensional representations attached to quadratic Dirichlet characters $\chi_{1}$ and $\chi_{2}$ satisfying $\chi_{1} \chi_{2}=\epsilon_{K}$, so that in particular $\chi_{1}(p)=-\chi_{2}(p)$. When the iterated integral of the left-hand side of (69) is nonzero, Conjecture ES predicts that precisely one of the characters $\chi_{1}$ or $\chi_{2}$ occurs in the Mordell-Weil group of $E$. Assume that it is $\chi_{1}$ which arises, and set $\iota:=\chi_{1}(p)= \pm 1$.

One then has

$$
P_{1} \in E(H)_{L}^{\chi_{1}} .
$$

Conjecture ES predicts that, for a suitable choice of test vectors,

$$
\int_{\breve{\gamma} \alpha} \breve{f} \cdot \breve{h} \stackrel{?}{=} \frac{\log _{E, p}\left(P_{1}\right) \log _{E, p}\left(P_{2}^{-\iota}\right)}{\log _{p}\left(u_{K}\right)} .
$$

Finally, consider the case in which $\psi_{1}^{2}=\psi_{2}^{2}=1$. The representation $V_{g h}$ then decomposes as a sum of one-dimensional summands, and Conjecture ES takes the form

$$
\int_{\breve{\gamma} \alpha} \breve{f} \cdot \breve{h} \stackrel{?}{=} \frac{\log _{E, p}\left(P_{1}\right) \log _{E, p}\left(P_{2}\right)}{\log _{p}\left(u_{K}\right)},
$$

provided that the points $P_{1}$ and $P_{2}$, which both belong to quadratic extensions of $\mathbb{Q}$, belong to distinct eigenspaces for $\sigma_{p}$; in the case where they are in the same eigenspace, Conjecture ES predicts a subtle vanishing of the $p$-adic iterated integral arising from a degeneracy in the regulator term. This case is illustrated in Example 4.3 below. 
4.2. Numerical evidence. Weight-one modular forms whose Artin representations can be induced only from characters of class groups of real quadratic fields are rather rare in small level. The first such occurrence is in level 145 , where one finds such a form coming from a quartic character of $\mathbb{Q}(\sqrt{5})$ with conductor a prime of norm 29. The next example is concerned with this modular form.

EXAMPLE 4.1. Let $K=\mathbb{Q}(\sqrt{5})$, and let

$$
\psi_{g}=\psi_{h}^{-1}
$$

be the quartic character of $K$ which is ramified at the prime $\lambda=(29$, $(11+\sqrt{5}) / 2)$ of norm 29. Let $\chi_{5}$ and $\chi_{29}$ be the quadratic and quartic Dirichlet characters of conductors 5 and 29 , respectively, and let $\chi:=\chi_{5} \chi_{29}$ be the odd quartic character of conductor 5.29. Both $\psi_{g}$ and $\chi$ take values in the field $L:=\mathbb{Q}(i)$ and are unique up to conjugation by $\operatorname{Gal}(L / \mathbb{Q})$. Fix a choice of these characters in such a way that the theta series

$$
g \in S_{1}\left(5 \cdot 29, \chi^{-1}\right), \quad h \in S_{1}(5 \cdot 29, \chi)
$$

associated to $\psi_{g}$ and $\psi_{h}$ belong to spaces of weight-one modular forms with those characters (and Fourier coefficients in $L$ ). The Artin representation $V_{g h}$ is given by

$$
V_{g h}=L \oplus L\left(\chi_{5}\right) \oplus V_{\psi},
$$

where $\psi$ is the unique (up to conjugation) quartic ring class character of $K$ of conductor 29. Let $H$ be the abelian quartic extension of $K$ cut out by $\psi$, and write $\operatorname{Gal}(H / K)=:\langle\sigma\rangle$. The field $H$ is Galois over $\mathbb{Q}$, with Galois group $G=D_{8}$, the dihedral group of order 8 . The subfield of $H$ fixed by the centre $\left\langle\sigma^{2}\right\rangle$ of $G$ is the totally real biquadratic field $M:=\mathbb{Q}(\sqrt{5}, \sqrt{29})$, and $H$ is the quadratic extension of $M$ given by

$$
H=M(\sqrt{\delta})=M\left(\sqrt{\delta^{\prime}}\right), \quad \delta=\left(\frac{-29+3 \sqrt{29}}{2}\right), \quad \delta^{\prime}=\left(\frac{-29-3 \sqrt{29}}{2}\right) .
$$

Note that $\delta$ and $\delta^{\prime}$ are totally negative elements of $M$, and that $\delta \delta^{\prime}=5 \cdot 29$.

Let $f$ be the weight-two cusp form of level 17 attached to the unique elliptic curve

$$
E: y^{2}+x y+y=x^{3}-x^{2}-x-14
$$

of conductor 17 denoted $17 a$ in the tables of Cremona. The conductor of $E$ is inert in the quadratic field $K$. An explicit calculation shows that

$$
E(\mathbb{Q})_{L}=0, \quad E(K)_{L}^{\chi_{5}}=L \cdot P_{K} \quad \text { where } P_{K}=\left(\frac{379}{20}, \frac{-1995+7218 \sqrt{5}}{200}\right) .
$$


The group $G$ admits four distinct one-dimensional representations, on which $\sigma^{2}$ acts trivially, and a unique irreducible representation of dimension two with rational traces, which is isomorphic to $V_{\psi}$ and on which $\sigma^{2}$ acts as multiplication by -1 . Hence

$$
E(H)_{\mathbb{Q}}^{V_{\psi}}=E(H)_{\mathbb{Q}}^{\sigma^{2}=-1} .
$$

Considerations involving signs in the functional equations of $L$-series suggest that the representation $V_{\psi}$ occurs with odd multiplicity in $E(H)$ (see for example the introduction of [Dar]). Note that the field $H$, a totally odd cyclic quartic extension of $K$, contains no imaginary quadratic subfields. The classical Heegner point construction is therefore unavailable to produce the global points in $E(H)_{\mathbb{Q}}^{V_{\psi}}$ whose existence is predicted by the Birch and Swinnerton-Dyer conjecture. The Stark-Heegner point algorithms described in [DP] can be used as a substitute for the method based on Heegner points, and they yield four nontrivial points in $E(H)$ given by

$$
\begin{aligned}
P= & \frac{-220777-17703 \sqrt{145}}{5800}, \frac{214977+17703 \sqrt{145}}{11600} \\
& \left.+\frac{28584525+3803103 \sqrt{5}+1645605 \sqrt{29}+2364771 \sqrt{145}}{290000} \sqrt{\delta}\right) \\
P^{\sigma}= & \frac{-220777+17703 \sqrt{145}}{5800}, \frac{214977-17703 \sqrt{145}}{11600} \\
& \left.+\frac{28584525+3803103 \sqrt{5}-1645605 \sqrt{29}-2364771 \sqrt{145}}{290000} \sqrt{\delta^{\prime}}\right) \\
P^{\sigma^{2}=} & \left(\frac{-220777-17703 \sqrt{145}}{5800}, \frac{214977+17703 \sqrt{145}}{11600}\right. \\
& \left.-\frac{28584525+3803103 \sqrt{5}+1645605 \sqrt{29}+2364771 \sqrt{145}}{290000} \sqrt{\delta}\right) \\
P^{\sigma^{3}=} & \left(\frac{-220777+17703 \sqrt{145}}{5800}, \frac{214977-17703 \sqrt{145}}{11600}\right. \\
& \left.-\frac{28584525+3803103 \sqrt{5}-1645605 \sqrt{29}-2364771 \sqrt{145}}{290000} \sqrt{\delta^{\prime}}\right) .
\end{aligned}
$$

These four Galois conjugate points are constructed from periods arising from a $p$-adic integration on $\mathcal{H}_{17} \times \mathcal{H}$ based on the minus modular symbol of $f$, as described in [Dar]. The conjectures of loc.cit. predict that these points should lie in the minus eigenspace for complex conjugation, which coincides in this case with the central involution $\sigma^{2}$ of $\operatorname{Gal}(H / \mathbb{Q})$. It can indeed be observed 
that $\sigma^{2} P=-P$ and that $\sigma^{3} P=-\sigma P$. In particular, the Stark-Heegner point $P$ and its conjugates generate a rank-2 subgroup of $E(H)$ which is isomorphic to $V_{\psi}$. This shows that $r\left(E, V_{\psi}\right) \geqslant 1$, and the belief that Stark-Heegner points ought to behave like their quadratic imaginary counterparts strongly suggests that in fact $r_{\text {an }}\left(E, V_{\psi}\right)=1$. If such is the case, Conjecture ES predicts the nonvanishing of some $p$-adic iterated integral attached to $(f, g, h)$.

The global point $P$ and its conjugates were obtained from a 17-adic calculation. This is an essential limitation of [Dar], where one is forced to work $p$-adically at a prime $p$ dividing the conductor of $E$. On the other hand, the $p$-adic iterated integral appearing on the left of (70) can be calculated using any prime $p$ which is inert in $K$. It turns out to be expedient (albeit, not essential, as in the calculations relying on the algorithms of [DP]) to work at $p=17$. This is because, with this choice of $p$, the classical modular form $f$ of level 17 is $p$-adically of level 1 , and the $p$-adic iterated integral can therefore be calculated by applying the ordinary projection algorithms of [La2] to a space of ordinary overconvergent 17 -adic modular forms of weight one and relatively modest level $5 \cdot 29$.

It can be checked that, for a suitable embedding of $H$ into the quadratic extension $\mathbb{Q}_{17^{2}}$ of $\mathbb{Q}_{17}$, the points $P$ and $\sigma^{2} P$ are defined over $\mathbb{Q}_{17}$, while the points $\sigma P$ and $\sigma^{3} P$ are defined over $\mathbb{Q}_{17^{2}}$ and are interchanged by the Frobenius element $\sigma_{17}$. Fix such an embedding from now on, and let $\gamma_{ \pm} \in S_{1}\left(\Gamma_{1}(5 \cdot 29)\right.$, $\chi)_{L}^{\vee}\left[g_{ \pm}\right]$be the unique element satisfying

$$
\gamma_{ \pm}\left(g_{ \pm}^{*}\right)=1
$$

Here, $g_{ \pm}=g_{ \pm \zeta_{8}^{3}}$ denotes the 17-stabilizations of $g$. A numerical evaluation of the 17 -adic iterated integrals, to 16 significant digits, shows that

$$
\int_{\gamma_{ \pm}} f \cdot h=1259389260500681328 \times 17 \bmod 17^{16},
$$

and that

$$
\int_{\gamma_{ \pm}} f \cdot h=\frac{\log _{E, 17}\left(P_{K}\right) \log _{E, 17}(P)}{3 \cdot 17 \cdot \log _{17}\left(u_{K}\right)}
$$

to an accuracy of 16 significant 17 -adic digits. Since $\sigma_{17} P_{K}=-P_{K}$, while $P$ generates $E(H)_{L}^{V_{\psi}} \cap E\left(\mathbb{Q}_{17}\right)$, this is in perfect agreement with the prediction of (70).

REMARK 4.2. It is worth emphasizing that the Stark-Heegner point $P$ is a priori quite mysterious and not known to correspond to a global point, while the 17-adic iterated integral appearing on the left-hand side of (72) can be 
related to the 17-adic logarithm of the global cohomology class $\kappa(\breve{f}, \breve{g}, \breve{h}) \in$ $H^{1}\left(H, V_{17}(E)\right)$ constructed in [DR2] as a limit of 17-adic étale Abel-Jacobi images of suitable 'generalized Gross-Schoen diagonal cycles'. Since both the 17-adic iterated integral appearing on the left-hand side of (72) and the 'integrals on $\mathcal{H}_{17} \times \mathcal{H}^{\prime}$ used to compute the Stark-Heegner point $P$ are given by explicit 17-adic analytic expressions, a proof of formula (72) does not seem entirely out of reach. Such a proof would represent substantial progress in understanding the global properties of Stark-Heegner points, perhaps making it possible to use them in Euler system arguments of the kind exploited originally by Kolyvagin. This would extend the approach described in [DR2, Theorems A and C] from 'rank-0' to certain 'rank-1' settings.

The next example involves forms $g$ and $h$ with representation having projective image $D_{4}$. Such odd representations are induced from class characters of both real and imaginary quadratic fields, so this example would be equally at home in Section 3. Its principal novelty is that it illustrates situations where the $p$-adic iterated integrals vanish in rank- $(1,1)$ settings because the regulator term of Conjecture ES is 0 .

EXAMPLE 4.3. Consider the curves

$$
\begin{aligned}
& E_{a}: y^{2}+y=x^{3}-x^{2}+10 x+6 \\
& E_{b}: y^{2}+x y+y=x^{3}+x^{2}-x-2 \\
& E_{c}: y^{2}+y=x^{3}-x^{2}-x+1
\end{aligned}
$$

of conductor 155 labelled $155 a, 155 b$, and $155 c$ in Cremona's tables, and write $f_{a}, f_{b}$, and $f_{c}$ for the associated weight-two newforms.

Let $\chi_{1}$ be the trivial character, let $\chi_{5}$ and $\chi_{31}$ denote the (even and odd, respectively) characters of conductor 5 and 31 , and set $\chi:=\chi_{155}:=\chi_{5} \cdot \chi_{31}$, which takes values in $L=\mathbb{Q}$. The space $S_{1}(155, \chi)$ has dimension three and contains three dihedral forms: one form $g$ with integer Fourier coefficients

$$
g=q+q^{4}-q^{5}-q^{9}+q^{16}-2 q^{19}-\cdots
$$

and a second pair of complex conjugate forms each defined over the cyclotomic field $\mathbb{Q}\left(\zeta_{3}\right)$. The form $g$ has associated linear representation $\rho_{g}$ with projective image $D_{4}$, and $\rho_{g}$ can be described in the following equivalent ways.

(1) It is induced from a quartic character of the Hilbert class field of the imaginary quadratic field $\mathbb{Q}(\sqrt{-155})$.

(2) It is induced from a quadratic character of the imaginary quadratic field $\mathbb{Q}(\sqrt{-31})$, whose conductor is a prime ideal of norm 5 . 
(3) It is induced from a quadratic character of the real quadratic field $\mathbb{Q}(\sqrt{5})$, whose conductor is a prime ideal of norm 31 .

(The remaining pair of complex conjugate forms are associated to representations which arise from quartic characters of $\mathbb{Q}(\sqrt{-31})$ with conductor a prime ideal of norm 5-they are not considered further in this example.)

The representation $V_{g g}$ decomposes as

$$
V_{g g}=L \oplus L\left(\chi_{5}\right) \oplus L\left(\chi_{31}\right) \oplus L\left(\chi_{155}\right),
$$

and the corresponding ranks are given by

$$
\begin{array}{llll}
r\left(E_{a} / \mathbb{Q}\right)=1, & r\left(E_{a}, \chi_{5}\right)=0, & r\left(E_{a}, \chi_{31}\right)=1, & r\left(E_{a}, \chi_{155}\right)=0 \\
r\left(E_{b} / \mathbb{Q}\right)=0, & r\left(E_{b}, \chi_{5}\right)=0, & r\left(E_{b}, \chi_{31}\right)=0, & r\left(E_{b}, \chi_{155}\right)=0 \\
r\left(E_{c} / \mathbb{Q}\right)=1, & r\left(E_{c}, \chi_{5}\right)=1, & r\left(E_{c}, \chi_{31}\right)=0, & r\left(E_{c}, \chi_{155}\right)=0,
\end{array}
$$

and likewise for the analytic ranks. In particular, the central critical value $L\left(E_{b}, \varrho_{g g}, 1\right)$ is nonzero, and hence the curve $E_{b}$ fails to satisfy Hypothesis B of the introduction. It will therefore be ignored from now on.

As predicted by the BSD conjecture, the points on $E_{a}$ and $E_{c}$ over $\mathbb{Q}$ and the fields $\mathbb{Q}(\sqrt{-31})$ and $\mathbb{Q}(\sqrt{5})$ are given by

$$
\begin{gathered}
P_{a}=(2,5), \quad P_{a, 31}=\left(\frac{-1630}{729}, \frac{40175 \sqrt{-31}-19683}{39366}\right), \\
P_{c}=(1,0), \quad P_{c, 5}=\left(0, \frac{-1+\sqrt{5}}{2}\right) .
\end{gathered}
$$

Consider both the primes $p=7$ and $p=13$, which have different splitting behaviours in the three quadratic fields, as shown in the table below.

\begin{tabular}{cccc}
\hline$p$ & $\chi_{5}(p)$ & $\chi_{31}(p)$ & $\chi_{155}(p)$ \\
\hline 7 & -1 & +1 & -1 \\
13 & -1 & -1 & +1 \\
\hline
\end{tabular}

For $p=7$, the eigenvalues of $\sigma_{p}$ on $V_{g}$ are $\alpha_{g}=1$ and $\beta_{g}=-1$, and so

$$
\left(\alpha_{g} \cdot \alpha_{g}, \alpha_{g} \cdot \beta_{g}\right)=(1,-1), \quad\left(\beta_{g} \cdot \alpha_{g}, \beta_{g} \cdot \beta_{g}\right)=(-1,1) .
$$

The 7-adic iterated integrals attached to $\left(f_{a}, \gamma_{ \pm 1}, g\right)$ and $\left(f_{c}, \gamma_{ \pm 1}, g\right)$ are

$$
\begin{gathered}
\int_{\gamma_{1}} f_{a} \cdot g=\int_{\gamma_{-1}} f_{a} \cdot g=0 \bmod 7^{30} \\
\int_{\gamma_{1}} f_{c} \cdot g=\int_{\gamma_{-1}} f_{c} \cdot g=1416540068067896281523634 \times 7 \bmod 7^{30} .
\end{gathered}
$$


For $p=13$, the eigenvalues of $\sigma_{p}$ on $V_{g}$ are $\alpha_{g}=i$ and $\beta_{g}=-i$, and so

$$
\left(\alpha_{g} \cdot \alpha_{g}, \alpha_{g} \cdot \beta_{g}\right)=(-1,1), \quad\left(\beta_{g} \cdot \alpha_{g}, \beta_{g} \cdot \beta_{g}\right)=(1,-1) .
$$

The 13-adic iterated integrals attached to $\left(f_{a}, \gamma_{ \pm i}, g\right)$ and $\left(f_{c}, \gamma_{ \pm i}, g\right)$ are

$$
\begin{gathered}
\int_{\gamma_{i}} f_{a} \cdot g=-\int_{\gamma_{-i}} f_{a} \cdot g=10546307292618612 \bmod 13^{15} \\
\int_{\gamma_{i}} f_{c} \cdot g=\int_{\gamma_{-i}} f_{c} \cdot g=21913581880269709 \bmod 13^{15}
\end{gathered}
$$

Following the notations in the rank- $(1,1)$ setting of Conjecture ES, the pair $\left(\varrho_{1}, \varrho_{2}\right)$ of irreducible subrepresentations of $V_{g g}$ attached to the curves $E_{a}$ and $E_{c}$ can be taken to be $\left(\chi_{1}, \chi_{31}\right)$ and $\left(\chi_{1}, \chi_{5}\right)$, respectively, where $\chi_{1}$ is the trivial character. Observe that the eigenvalues of $\sigma_{13}$ are $(1,-1)$ in both cases. One checks to 15 digits of 13 -adic precision that

$$
\int_{\gamma_{i}} f_{a} \cdot g=\frac{80 \cdot \log _{E_{a}, 13}\left(P_{a}\right) \log _{E_{a}, 13}\left(P_{a, 31}\right)}{i \cdot 63 \cdot 13 \cdot \log _{13}\left(u^{-}\right)},
$$

where $i$ is the unique square root of -1 in $\mathbb{Z}_{13}$ which is $\equiv 5 \bmod 13$, and $u^{-}=$ $(1+\sqrt{5}) / 2$ is a fundamental unit for $\mathbb{Q}(\sqrt{5})$. Note that the same formula holds if one replaced $i$ by $-i$ on both sides. Similarly one checks to the same amount of 13 -adic precision that

$$
\int_{\gamma_{i}} f_{c} \cdot g=-\frac{80 \cdot \log _{E_{c}, 13}\left(P_{c}\right) \log _{E_{c}, 13}\left(P_{c, 5}\right)}{21 \cdot 13 \cdot \log _{13}\left(u^{-}\right)} .
$$

Turning now to $p=7$ and the curve $E_{a}$, observe that $\left(\chi_{1}(7), \chi_{31}(7)\right)=(1,1)$, and hence the regulator term in Conjecture ES vanishes. This explains why the iterated integrals attached to $\left(f_{a}, \gamma_{ \pm 1}, g\right)$ were observed to vanish, at least numerically to 30 digits of 7 -adic precision. The situation for the curve $E_{c}$ is different, since $\left(\chi_{1}(7), \chi_{5}(7)\right)=(1,-1)$. As predicted by Conjecture ES, the authors were able to verify, to 30 digits of 7 -adic precision, that

$$
\int_{\gamma_{ \pm 1}} f_{c} \cdot g=-\frac{8 \cdot \log _{E_{c}, 7}\left(P_{c}\right) \log _{E_{c}, 7}\left(P_{c, 5}\right)}{3 \cdot 7 \cdot \log _{7}\left(u^{-}\right)} .
$$

The final three sections summarize some of the numerical evidence that was obtained in support of Conjecture ES in settings where $g$ and $h$ are not both theta series. It is an apt moment to pause momentarily and thank again Steve Donnelly: most of the interesting global points in these sections were found by him upon 
our request using his descent code. We might also stress here that although the overconvergent projection algorithms used to compute $p$-adic iterated integrals are efficient, such computations still remain something of a technological tour de force: many of our examples pushed the available hardware and software (the algorithms of [La2] in particular) to their current limits.

\section{Stark points over $A_{4}, S_{4}$ or $A_{5}$ extensions and exotic weight-one forms}

This section focusses on the case where $V_{g h}$ is reducible and at least one of $g$ or $h$ is an exotic cusp form whose associated projective Galois representation cuts out a nontotally real extension of $\mathbb{Q}$ with Galois group $A_{4}, S_{4}$, or $A_{5}$. (The case in which $V_{g h}$ is irreducible is very difficult to test experimentally, due to the apparent scarcity of such $V_{g h}$ of manageable size occurring with multiplicity two in the Mordell-Weil group of elliptic curves of reasonable conductor. See [DLR1, Example 6.2] though for a successful experiment in the analogous setting in which Mordell-Weil groups are replaced by groups of units.)

LEMMA 5.1. Assume that one of $g$ or $h$ is an exotic weight-one form and that $g$ and $h$ satisfy the self-duality condition. Then the representation $V_{g h}$ is irreducible, unless $g$ is the twist of $h^{*}$ by a quadratic character $\iota$, in which case

$$
V_{g h}=L(\iota) \oplus \operatorname{Ad}_{g}(\iota) .
$$

Proof. The discussion in [DLR1, Section 2] rules out the case where $V_{g h}$ contains an irreducible subrepresentation of dimension two. Hence $V_{g h}$ contains an irreducible one-dimensional subrepresentation, and the result follows from Schur's lemma (see [DLR1, Section 2]).

So if $V_{g h}$ is reducible, Lemma 5.1 implies that $g$ is a quadratic twist of $h^{*}$. Since it is always possible to replace the form $f$ by one of its quadratic twists, there is no real loss in generality in assuming that $g=h^{*}$, which is what shall be done in the rest of this section. In this setting, Conjecture ES suggests a new, efficiently computable (conjectural) analytic formula for the $p$-adic logarithm of global points defined over the $A_{4}, S_{4}$, or $A_{5}$-extension cut out by the adjoint $\mathrm{Ad}_{g}$ (or, equivalently, by the projective representation attached to $g$ ).

5.1. Theoretical preliminaries. Let $h$ be an exotic weight-one form, let $g=h^{*}$ be its conjugate form, and let $L$ be as before the field generated by the Fourier coefficients of $h$, or of $g=h^{*}$. The representation $V_{g h}=L \oplus \operatorname{Ad}_{g}$, where $L$ refers to the trivial representation of $G_{\mathbb{Q}}$ with coefficients in $L$, factors 
through $\mathrm{Gal}(H / \mathbb{Q})$, where $H$ is the field cut out by the projective representation attached to $g$, so that

$$
\operatorname{Gal}(H / \mathbb{Q}) \simeq A_{4}, S_{4} \text { or } A_{5} .
$$

Conjecture ES predicts that the trilinear form $I_{p}^{\prime}$ in (8) arising from $p$-adic iterated integrals may be nonidentically zero only when

$$
r(E, L)=r\left(E, \operatorname{Ad}_{g}\right)=1,
$$

or

$$
r(E, L)=0, \quad r\left(E, \mathrm{Ad}_{g}\right)=2 .
$$

In the rank- $(1,1)$ setting, Conjecture ES asserts that, for a suitable choice of elements

$$
P_{\mathbb{Q}} \in E(\mathbb{Q}), \quad P_{g_{\alpha}} \in\left(E(H)_{L}^{\mathrm{Ad}_{g}}\right)^{\sigma_{p}=\alpha_{g} / \beta_{g}}, \quad u_{g_{\alpha}} \in\left(\left(\mathcal{O}_{H}^{\times}\right)_{L}^{\mathrm{Ad}_{g}}\right)^{\sigma_{p}=\alpha_{g} / \beta_{g}},
$$

which, since $\alpha_{g} \neq \pm \beta_{g}$, are unique up to scaling by $L^{\times}$, and for a suitable choice $\breve{f}, \breve{\gamma}_{g_{\alpha}}$, and $\breve{h}$ of test vectors attached to $\left(f, g_{\alpha}, h\right)$,

$$
\int_{\breve{\gamma}_{g \alpha}} \breve{f} \cdot \breve{h} \stackrel{?}{=} \frac{\log _{E, p}\left(P_{\mathbb{Q}}\right) \log _{E, p}\left(P_{g_{\alpha}}\right)}{\log \left(u_{g_{\alpha}}\right)} .
$$

In the rank-2 setting, Conjecture ES asserts that

$$
\int_{\breve{\gamma}_{g \alpha}} \breve{f} \cdot \breve{h} \stackrel{?}{=} \frac{\log _{E, p}\left(P_{1}\right) \log _{E, p}\left(Q_{g_{\alpha}}\right)-\log _{E, p}\left(Q_{1}\right) \log _{E, p}\left(P_{g_{\alpha}}\right)}{\log \left(u_{g_{\alpha}}\right)},
$$

where $(P, Q)$ is a basis of $\operatorname{Hom}_{G_{\mathbb{Q}}}\left(\operatorname{Ad}_{g}, E(H)_{L}\right)$, and $P_{1}, Q_{1}$ (respectively $P_{g_{\alpha}}$, $Q_{g_{\alpha}}$ ) denote the images of a vector $v_{1} \in \operatorname{Ad}_{g}^{\sigma_{p}=1}$ (respectively, $v_{2} \in \operatorname{Ad}_{g}^{\sigma_{p}=\alpha_{g} / \beta_{g}}$ ) under these homomorphisms.

In the rank- $(1,1)$ setting, the conjectural formula above gives an explicit, albeit conjectural, $p$-adic analytic expression for a global point-namely $P_{g_{\alpha}}-$ defined over the field $H$ cut out by $\mathrm{Ad}_{g}$, depending on quantities which are a priori accessible computationally:

$$
P_{g_{\alpha}} \stackrel{?}{=} \exp _{E, p}\left(\frac{\log \left(u_{g_{\alpha}}\right)}{\log _{E, p}\left(P_{\mathbb{Q}}\right)} \cdot \int_{\breve{\gamma}_{g \alpha}} \breve{f} \cdot \breve{h}\right) .
$$

The point $P_{g_{\alpha}}$ can be viewed as an elliptic curve analogue of the Stark unit $u_{g_{\alpha}}$. For the sake of numerical experiments, it is worth giving a more explicit description of $P_{g_{\alpha}}$, depending on the nature of $\operatorname{Gal}(H / \mathbb{Q})$. 
5.1.1. Cases where $g$ is of tetrahedral type. The group $G=\operatorname{Gal}(H / \mathbb{Q}) \simeq A_{4}$ has three representations of dimension one which factor through the quotient $A_{4} / V_{4} \simeq A_{3}$, and a unique faithful irreducible representation of dimension three, which is therefore isomorphic to the adjoint representation $\operatorname{Ad}_{g}$. Let $R(x)$ be any degree-four polynomial whose splitting field is $H$, and let $M$ be the quartic field which is generated by a single root of $R$. Then $\operatorname{Ad}_{g}$ is related to a permutation representation by the formula

$$
\operatorname{Ad}_{g}=\operatorname{Ind}_{G_{M}}^{G_{\mathbb{Q}}} L-L .
$$

It follows that

$$
r\left(E, \operatorname{Ad}_{g}\right)=r\left(E, \operatorname{Ind}_{M}^{\mathbb{Q}} L\right)-r(E, L)=\operatorname{rank}(E(M))-\operatorname{rank}(E(\mathbb{Q})) .
$$

(The Artin formalism for $L$-series implies similar equalities for the analytic ranks.) In particular when $r\left(E, \mathrm{Ad}_{g}\right)=1$, there is (up to scaling) a unique point $P \in E(M)$ whose trace to $E(\mathbb{Q})$ is zero, and $E(H)_{L}^{\mathrm{Ad}_{g}}$ is generated, as an $L$ vector space, by the four Galois translates of $P$, whose sum is zero. Let $P=: P_{1}$, $P_{2}, P_{3}$, and $P_{4}$ denote these Galois translates, on which $\operatorname{Gal}(H / \mathbb{Q})=A_{4}$ acts via the natural permutation action. The point $P_{g_{\alpha}}$ can be expressed as a linear combination of the $P_{j}$, depending on the conjugacy class of $\sigma_{p}$.

Case 1: $\sigma_{p}=1$. In the case when $\alpha_{g} / \beta_{g}=\beta_{g} / \alpha_{g}=1$, the representations $\varrho_{g}$ and $\mathrm{Ad}_{g}$ are not regular at $p$, and Hypothesis $\mathrm{C}$ is not satisfied.

Case 2: $\sigma_{p}$ is of order 2. In that case it may be assumed that the points $P_{1}$ and $P_{2}$ are defined over $\mathbb{Q}_{p^{2}}$ and are interchanged by $\sigma_{p}$, and likewise for $P_{3}$ and $P_{4}$. It follows that $\alpha_{g} / \beta_{g}=-1$, and that

$$
\begin{aligned}
& \left(E(H)_{L}^{\mathrm{Ad}_{g}}\right)^{\sigma_{p}=1}=L \cdot\left(P_{1}+P_{2}\right)=L \cdot\left(P_{3}+P_{4}\right) \quad \text { is one dimensional, } \\
& \left(E(H)_{L}^{\mathrm{Ad}_{g}}\right)^{\sigma_{p}=-1}=L \cdot\left(P_{1}-P_{2}\right) \oplus L \cdot\left(P_{3}-P_{4}\right) \quad \text { is two dimensional. }
\end{aligned}
$$

In this scenario, in which $V_{g}$ is regular but $\mathrm{Ad}_{g}$ is not, the point $P_{g_{\alpha}}$ is a specific $L$-linear combination of the points $P_{1}-P_{2}$ and $P_{3}-P_{4}$, which lies in the welldefined one-dimensional subspace hom $\left(V_{g}^{g_{\beta}}, V_{g}^{g_{\alpha}}\right)$ of $\operatorname{Ad}_{g}$.

Case 3: $\sigma_{p}$ is of order 3. In that case, it may be assumed that $\sigma_{p}$ fixes the point $P_{1}$, which is thus defined over $\mathbb{Q}_{p}$, and cyclically permutes the points $P_{2}, P_{3}$, and $P_{4}$. The eigenvalues of $\sigma_{p}$ on $\operatorname{Ad}_{g}$ are then equal to $1, \alpha_{g} / \beta_{g}=\zeta_{3}$, and $\beta_{g} / \alpha_{g}=\zeta_{3}^{-1}$, for some primitive cube root $\zeta_{3}$ of 1 , and $P_{g_{\alpha}}$ is determined, up to scaling, by the rule

$$
P_{g_{\alpha}}=P_{2}+\zeta_{3}^{-1} P_{3}+\zeta_{3} P_{4}
$$


In particular,

$$
\left(E(H)_{L}^{\mathrm{Ad}_{g}}\right)^{\sigma_{p}=\alpha_{g} / \beta_{g}}=L \cdot P_{g_{\alpha}} \quad \text { is one dimensional. }
$$

This is the only regular case, in which the point $P_{g_{\alpha}}$ is completely pinned down by its specified behaviour under the action of $\sigma_{p}$.

REMARK 5.2. There are two distinct conjugacy classes of elements of order 3 in $A_{4}$, which are rational over $\mathbb{Q}\left(\zeta_{3}\right)$ and interchanged by the action of $\operatorname{Gal}\left(\mathbb{Q}\left(\zeta_{3}\right) / \mathbb{Q}\right)$. Note that 8 of the 12 elements in $A_{4}$ are of order 3 , and hence, by the Chebotarev density theorem, the Frobenius element $\sigma_{p}$ is regular for $\operatorname{Ad}_{g}$ for a set of primes $p$ of Dirichlet density $2 / 3$.

5.1.2. Cases where $g$ is of octahedral type. The dimensions of the irreducible representations of the group $G=\operatorname{Gal}(H / \mathbb{Q}) \simeq S_{4}$ are $1,1,2,3$, and 3 . The first two representations are the trivial and sign representations, and the two-dimensional representation factors through the quotient $S_{4} / V_{4}=S_{3}$. In particular, the only faithful irreducible representations of $G$ are three dimensional, and there are two such, whose characters are given in the following table.

\begin{tabular}{rccccc}
\hline Size & 1 & 6 & 3 & 8 & 6 \\
Class & 1 & $(12)$ & $(12)(34)$ & $(123)$ & $(1234)$ \\
\hline$\varrho_{1}$ & 3 & 1 & -1 & 0 & -1 \\
$\varrho_{2}$ & 3 & -1 & -1 & 0 & 1 \\
\hline
\end{tabular}

The representation $\varrho_{1}$ occurs in the natural permutation representation of $G$ on four letters, while $\varrho_{2}$ is just the twist of $\varrho_{1}$ by the sign character. Among the two representations, it is $\varrho_{2}$ which is the adjoint representation. This can be seen by noting that the eigenvalues of $\varrho_{1}((1234))$ are visibly $-1, i$, and $-i$, and that those of $\varrho_{2}((1234))$ are $1, i$, and $-i$, and that only the latter set of eigenvalues is consistent with the property of the adjoint representation that all elements in $G$ admit 1 as eigenvalue.

Let $R(x)$ be any degree-four polynomial whose splitting field is $H$, and let $M$ be the quartic field which is generated by a single root of $R$. The field $H$ is an $S_{3}$-extension of $M$, and hence there is a unique quadratic extension $\tilde{M}$ of $M$ contained in $H$. The field $H$ also contains a unique quadratic field $K / \mathbb{Q}$, which is cut out by the sign character of $S_{4}$ and satisfies $\tilde{M}=M K$. The representation $\mathrm{Ad}_{g}$ is described by the formula

$$
\operatorname{Ad}_{g}=\operatorname{Ind}_{G_{M}}^{G_{\mathbb{Q}}} \operatorname{sgn}-\mathrm{sgn},
$$


where sgn is viewed both as the quadratic character of $G_{M}$ and $G_{\mathbb{Q}}$ induced by the sign characters on the finite quotients $\operatorname{Gal}(H / M) \simeq S_{3}$ and $\operatorname{Gal}(H / \mathbb{Q}) \simeq S_{4}$. It follows that

$$
r\left(E, \operatorname{Ad}_{g}\right)=r\left(E, \operatorname{Ind}_{M}^{H} \operatorname{sgn}\right)-r(E, \operatorname{sgn})=\operatorname{dim}_{L} E(\tilde{M})_{L}^{-}-\operatorname{dim}_{L} E(K)^{-},
$$

where the superscript - refers to the -1 eigenspace for the involutions in $\operatorname{Gal}(\tilde{M} / M)$ and $\operatorname{Gal}(K / \mathbb{Q})$, respectively. When $r\left(E, \operatorname{Ad}_{g}\right)=1$, there is, by Frobenius reciprocity, a unique point $P \in E(\tilde{M})^{-}$, up to scaling. Let $P_{1}, P_{2}, P_{3}$, and $P_{4}$ denote the $A_{4}$ translates of $P=P_{1}$, and observe that $\sigma \in G=S_{4}$ acts on these points by the rule

$$
\sigma P_{j}=\left\{\begin{aligned}
P_{\sigma j} & \text { if } \sigma \in A_{4} \\
-P_{\sigma j} & \text { if } \sigma \in S_{4}-A_{4} .
\end{aligned}\right.
$$

The resulting explicit description of the action of $G$ on these four points, which together generate $E(H)_{L}^{\mathrm{Ad}_{g}}$, makes it possible to describe the point $P_{g_{\alpha}}$ explicitly, depending on the conjugacy class of $\sigma_{p}$ in $G$.

Case 1: $\sigma_{p}=1$. In that case, $\alpha_{g}=\beta_{g}$, and hence Hypothesis $\mathrm{C}^{\prime}$ is not satisfied.

Case 2: $\sigma_{p}$ is of order 2 and odd; that is, it is conjugate to the transposition (12). In that case, after relabelling the $P_{j}$, it can be assumed that

$$
\sigma_{p} P_{1}=-P_{2}, \quad \sigma_{p} P_{2}=-P_{1}, \quad \sigma_{p} P_{3}=-P_{3}, \quad \sigma_{p} P_{4}=-P_{4} .
$$

In particular, the $x$-coordinates of the points $P_{1}$ and $P_{2}$ lie in $\mathbb{Q}_{p^{2}}$ and are permuted by $\operatorname{Gal}\left(\mathbb{Q}_{p^{2}} / \mathbb{Q}_{p}\right)$, while the $x$-coordinates of $P_{3}$ and $P_{4}$ are defined over $\mathbb{Q}_{p}$, even though all four points are defined over $\mathbb{Q}_{p^{2}}$. The eigenvalues of $\sigma_{p}$ are equal to 1 and $-1=\alpha_{g} / \beta_{g}=\beta_{g} / \alpha_{g}$, and

$$
\begin{gathered}
\left(E(H)_{L}^{\mathrm{Ad}_{g}}\right)^{\sigma_{p}=1}=L \cdot\left(P_{1}-P_{2}\right) \quad \text { is one dimensional, } \\
\left(E(H)_{L}^{\mathrm{Ad}_{g}}\right)^{\sigma_{p}=-1}=L \cdot P_{3} \oplus L \cdot P_{4} \quad \text { is two dimensional. }
\end{gathered}
$$

This is another irregular case, in which the point $P_{g_{\alpha}}$ is a specific $L$-linear combination of $P_{3}$ and $P_{4}$ lying in the line hom $\left(V_{g}^{g_{\beta}}, V_{g}^{g_{\alpha}}\right)$ of $\operatorname{Ad}_{g}$.

Case 3: $\sigma_{p}$ is of order 2 and even; that is, it is conjugate to the permutation (12)(34). After eventually relabelling the $P_{j}$, it can be assumed that

$$
\sigma_{p} P_{1}=P_{2}, \quad \sigma_{p} P_{2}=P_{1}, \quad \sigma_{p} P_{3}=P_{4}, \quad \sigma_{p} P_{4}=P_{3},
$$

so that

$$
\left(E(H)_{L}^{\mathrm{Ad}_{g}}\right)^{\sigma_{p}=1}=L \cdot\left(P_{1}+P_{2}\right)=L \cdot\left(P_{3}+P_{4}\right) \quad \text { is one dimensional, }
$$




$$
\left(E(H)_{L}^{\operatorname{Ad}_{g}}\right)^{\sigma_{p}=-1}=L \cdot\left(P_{1}-P_{2}\right) \oplus L \cdot\left(P_{3}-P_{4}\right) \quad \text { is two dimensional. }
$$

This is yet another irregular case, and $P_{g_{\alpha}}$ is some unspecified $L$-linear combination of $P_{1}-P_{2}$ and $P_{3}-P_{4}$.

Case 4: $\sigma_{p}$ is of order 3. In that case, it can be assumed that $\sigma_{p}$ fixes the point $P_{1}$, which is defined over $\mathbb{Q}_{p}$, and permutes the points $P_{2}, P_{3}$, and $P_{4}$ cyclically. The eigenvalues of $\sigma_{p}$ on $\mathrm{Ad}_{g}$ are then equal to $1, \alpha_{g} / \beta_{g}=\zeta_{3}$, and $\beta_{g} / \alpha_{g}=\zeta_{3}^{-1}$, and $P_{g_{\alpha}}$ is determined, up to scaling, by the rule

$$
P_{g_{\alpha}}=P_{2}+\zeta_{3}^{-1} P_{3}+\zeta_{3} P_{4} .
$$

In particular,

$$
\left(E(H)_{L}^{\mathrm{Ad}_{g}}\right)^{\sigma_{p}=\alpha_{g} / \beta_{g}}=L \cdot P_{g_{\alpha}} \quad \text { is one dimensional. }
$$

Case 5: $\sigma_{p}$ is of order 4, and hence is conjugate to (1234). In that case, it can be assumed that

$$
\sigma_{p} P_{1}=-P_{2}, \quad \sigma_{p} P_{2}=-P_{3}, \quad P_{3}=-P_{4}, \quad \sigma P_{4}=-P_{1} .
$$

The eigenvalues of $\sigma_{p}$ are equal to $1, i$, and $-i$, and

$$
\begin{aligned}
\left(E(H)_{L}^{\mathrm{Ad}_{g}}\right)^{\sigma_{p}=1} & =L \cdot\left(P_{1}-P_{2}+P_{3}-P_{4}\right), \\
\left(E(H)_{L}^{\mathrm{Ad}_{g}}\right)^{\sigma_{p}=i} & =L \cdot\left(P_{1}+i P_{2}-P_{3}-i P_{4}\right), \\
\left(E(H)_{L}^{\mathrm{Ad}_{g}}\right)^{\sigma_{p}=-i} & =L \cdot\left(P_{1}-i P_{2}-P_{3}+i P_{4}\right) .
\end{aligned}
$$

In particular, the $\alpha_{g} / \beta_{g}$-eigenspace for $\sigma_{p}$ is one dimensional if and only if $\sigma_{p}$ has order $>2$, which occurs for 14 of the 24 permutations in $S_{4}$. This regularity condition is therefore satisfied for a set of primes $p$ of density $7 / 12$.

5.1.3. Cases where $g$ is of icosahedral type. The dimensions of the irreducible representations of the group $G=\operatorname{Gal}(H / \mathbb{Q}) \simeq A_{5}$ are $1,3,3,4$, and 5, and the characters of the two distinct three-dimensional representations are described in the following table.

\begin{tabular}{rccccc}
\hline Size & 1 & 15 & 20 & 12 & 12 \\
Class & 1 & $(12)(34)$ & $(123)$ & $(12345)$ & $(12354)$ \\
\hline$\varrho_{1}$ & 3 & -1 & 0 & $\frac{1+\sqrt{5}}{2}$ & $\frac{1-\sqrt{5}}{2}$ \\
$Q_{2}$ & 3 & -1 & 0 & $\frac{1-\sqrt{5}}{2}$ & $\frac{1+\sqrt{5}}{2}$ \\
\hline
\end{tabular}


In this case, both the representations $\varrho_{1}$ and $\varrho_{2}$ arise as the adjoint of the Artin representation $\varrho_{g}$ and $\varrho_{g}^{\prime}$, respectively, where $\varrho_{g}^{\prime}$ denotes the Artin representation of the conjugate form of $g$, whose Fourier coefficients lie in the field $\mathbb{Q}(\sqrt{5})$.

It turns out to be easier to describe the direct sum $\varrho_{1} \oplus \varrho_{2}$ in Galois-theoretic terms rather than each $\varrho_{i}$ individually. Let $D_{10}$ be the dihedral group of order 10 , embedded naturally in $A_{5}$, and let $M$ be the non-Galois sextic field fixed by $D_{10}$. The field $M$ is generated by a root of a sextic polynomial $R(x)$, the so-called Lagrange sextic resolvent attached to the $A_{5}$ extension. Since $\mathrm{Gal}(H / M)=D_{10}$, there is a unique quadratic extension $\tilde{M}$ of $M$ contained in $H$, which is cut out by the unique nontrivial quadratic character $\chi^{-}$of $D_{10}$. It is not hard to check the identity of virtual representations:

$$
\varrho_{1} \oplus \varrho_{2}=\operatorname{Ind}_{G_{M}}^{G_{\mathbb{Q}}} \chi^{-},
$$

which implies that the six-dimensional representation $E(H)_{L}^{\mathrm{Ad}_{g}} \oplus E(H)_{L}^{W_{g^{\prime}}}$ is generated as an $L$-vector space, by the unique point $P \in E(\tilde{M})^{-}$together with its 12 Galois translates

$$
P=: P_{1}, P_{2}, P_{3}, P_{4}, P_{5}, P_{6}, \quad-P_{1},-P_{2},-P_{3},-P_{4},-P_{5},-P_{6} .
$$

The Stark point $P_{g_{\alpha}}$ is a $\mathbb{Q}(\sqrt{5})$-linear combination of the six linearly independent points $P_{j}$ with $1 \leqslant j \leqslant 6$. The coefficients that enter in this linear combination are completely determined by the requirement that $\sigma_{p} P_{g_{\alpha}}=$ $\alpha_{g} / \beta_{g} P_{g_{\alpha}}$, provided that the Frobenius element $\sigma_{p}$ is not of order $\leqslant 2$. Since $A_{5}$ has precisely 44 elements of order $>2$, this condition holds for a set of primes of Dirichlet density 11/15.

5.2. Numerical evidence. In contrast to the situation in Sections 4.2 and 7.2, numerical evidence is somewhat more bountiful in the setting of exotic modular forms. In particular, despite the appellation 'exotic' such forms seem to be more plentiful in small level than those studied in Section 4.2. (Note that in all our exotic examples we checked that Hypotheses A, B and C hold.)

As in Example 3.11, it is helpful to consider algebraic factors similar to those defined in [DR1, Theorem 3.1], given by

$$
\begin{aligned}
\mathcal{E}\left(g_{\alpha}, f, h\right):= & \left(1-\alpha_{g} \alpha_{f} \alpha_{h} p^{-1}\right) \times\left(1-\alpha_{g} \alpha_{f} \beta_{h} p^{-1}\right) \\
& \times\left(1-\alpha_{g} \beta_{f} \alpha_{h} p^{-1}\right) \times\left(1-\alpha_{g} \beta_{f} \beta_{h} p^{-1}\right) \\
\mathcal{E}_{0}\left(g_{\alpha}\right):= & \left(1-\alpha_{g}^{2} \chi^{-1}(p)\right) \\
\mathcal{E}_{1}\left(g_{\alpha}\right):= & \left(1-\alpha_{g}^{2} \chi^{-1}(p) p^{-1}\right) .
\end{aligned}
$$


Here, when $p$ divides the level of $f$, we set $\alpha_{f}=a_{p}(f)$ and $\beta_{f}=0$, and otherwise define these so that $x^{2}-a_{p}(f) x+p=\left(x-\alpha_{f}\right)\left(x-\beta_{f}\right)$. Economizing on notation, we define $\mathcal{E}_{\alpha}$ as

$$
\mathcal{E}_{\alpha}:=\frac{\mathcal{E}\left(g_{\alpha}, f, h\right)}{\mathcal{E}_{0}\left(g_{\alpha}\right) \mathcal{E}_{1}\left(g_{\alpha}\right)} \in L .
$$

We define $\mathcal{E}_{\beta}$ similarly, switching the roles of $\alpha_{g}$ and $\beta_{g}$ in the above.

Our first exotic example involves the tetrahedral form of level 133 first constructed by Tate.

EXAMPLE 5.3. Let $\chi$ be a Dirichlet character of level $133=7 \cdot 19$ of order 2 at 7 and order 3 at 19. (There are two such characters which are Galois conjugate to each other.) Denote by $\zeta$ a primitive 12th root of unity, and define $\zeta_{3}:=\zeta^{-4}$, a primitive cube root of unity. Then $S_{1}(133, \chi)$ is defined over $\mathbb{Q}\left(\zeta_{3}\right)$ and has dimension two. It contains two eigenforms defined over $\mathbb{Q}(\zeta)$, namely

$$
\begin{aligned}
h= & q+\left(\zeta^{2}-1\right) q^{2}-\zeta q^{3}+\zeta q^{5}+\left(-\zeta^{3}+\zeta\right) q^{6} \\
& +\zeta^{3} q^{7}-q^{8}+\left(\zeta^{3}-\zeta\right) q^{10}+\left(-\zeta^{3}+\zeta\right) q^{13}-\cdots
\end{aligned}
$$

and its Galois conjugate obtained by replacing $\zeta$ by $-\zeta$. Let $g=h^{*}$, the twist of $h$ by $\chi^{-1}$ (this has Fourier coefficients complex conjugate to those of $h$ ). The $A_{4}$ extension $H$ cut out by the projective representation attached to $g$ is the normal closure of the quartic field

$$
M:=\mathbb{Q}(w), \quad w^{4}+3 w^{2}-7 w+4=0,
$$

which has discriminant $133^{2}$.

Consider the elliptic curves

$$
\begin{aligned}
& E_{a}: y^{2}+y=x^{3}+x \\
& E_{b}: y^{2}+y=x^{3}+x^{2}-7 x+5
\end{aligned}
$$

of conductor $91=7 \cdot 13$ labelled $91 a$ and $91 b$ in Cremona's tables, and write $f_{a}$ and $f_{b}$ for the associated weight-two newforms. These curves both satisfy the conditions in the rank- $(1,1)$ setting of Conjecture ES, namely

$$
r\left(E_{a}, 1\right)=1, \quad r\left(E_{a}, \operatorname{Ad}_{g}\right)=1, \quad r\left(E_{b}, 1\right)=1, \quad r\left(E_{b}, \operatorname{Ad}_{g}\right)=1 .
$$

Let $P_{\mathbb{Q}}^{(a)}$ and $P_{\mathbb{Q}}^{(b)}$ denote Mordell-Weil generators of $E_{a}(\mathbb{Q})$ and $E_{b}(\mathbb{Q})$, respectively (modulo the torsion for $91 b$ ), and let $P^{(a)}$ and $P^{(b)}$ denote generators of the one-dimensional subspaces of $E_{a}(M)_{\mathbb{Q}}$ and $E_{b}(M)_{\mathbb{Q}}$, respectively, 
consisting of points with trace zero. A combination of naive search and twodescent shows that one can take

$$
\begin{aligned}
& P_{\mathbb{Q}}^{(a)}=(0,0), \\
& P^{(a)}=\left(\frac{-156 w^{3}-64 w^{2}-598 w+654}{289}, \frac{823 w^{3}+1464 w^{2}+5515 w-7663}{4913}\right), \\
& P_{\mathbb{Q}}^{(b)}=(-1,3), \\
& P^{(b)}=\left(9 w^{3}+5 w^{2}+31 w-45,-w^{3}+16 w^{2}+16 w+83\right) .
\end{aligned}
$$

Letting $p=13$, the Hecke polynomial $t^{2}-a_{13}(h) t+\chi(13)$ has roots $\beta_{h}=\zeta$ and $\alpha_{h}=-\zeta^{3}$. Hence the two 13-stabilizations $g_{\alpha}$ and $g_{\beta}$ of $g$ with $U_{p^{-}}$ eigenvalue $\alpha_{g}=\overline{\beta_{h}}=\zeta^{-1}$ and $\beta_{g}=\overline{\alpha_{h}}=\zeta^{3}$ are distinct. (Note that we are twisting by $\chi^{-1}$ here, and that $\chi^{-1}(p) \beta_{h}=\left(\alpha_{h} \beta_{h}\right)^{-1} \beta_{h}=\overline{\alpha_{h}}$, and likewise $\chi^{-1}(p) \alpha_{h}=\overline{\beta_{h}}$.) Let $\gamma_{g_{\alpha}}$ and $\gamma_{g_{\beta}}$ denote the corresponding dual elements.

Choose the embedding of $\mathbb{Q}(\zeta)$ into $\mathbb{Q}_{13}$ which sends $\zeta$ to the unique 12 th root of unity in $\mathbb{Z}_{13}$ which is congruent to 6 modulo 13 . The values of the $p$-adic iterated integrals, evaluated to 20 digits of 13-adic accuracy, are then given by the four quantities

$$
\begin{aligned}
& \int_{\gamma_{g \alpha}} f_{a} \cdot h=-6603156733219298602528 \bmod 13^{20} \\
& \int_{\gamma_{g_{\beta}}} f_{a} \cdot h=2705941994573919260213 \bmod 13^{20} \\
& \int_{\gamma_{g \alpha}} f_{b} \cdot h=-679336918469999467881 \times 13 \bmod 13^{20} \\
& \int_{\gamma_{g \beta}} f_{b} \cdot h=-2983964554989928769557 \bmod 13^{20} .
\end{aligned}
$$

The ratio $\alpha_{g} / \beta_{g}=\zeta^{-4}=\zeta_{3}$ is a primitive cube root of unity, and hence the eigenvalues of $\sigma_{13}$ on $\operatorname{Ad}_{g}$ are $1, \zeta_{3}$, and $\zeta_{3}^{2}$. The fact that $\sigma_{13}$ has order 3 puts us in Case 3 in the analysis in Section 5.1.1. Let

$$
P_{g_{\alpha}}^{(a)}, P_{g_{\beta}}^{(a)} \in E_{a}(H)_{L}^{\mathrm{Ad}_{g}}, \quad P_{g_{\alpha}}^{(b)}, P_{g_{\beta}}^{(b)} \in E_{b}(H)_{L}^{\mathrm{Ad}_{g}}
$$

be the points constructed from $P^{(a)}$ and $P^{(b)}$ using (76). The units $u_{g_{\alpha}}$ and $u_{g_{\beta}}$ are constructed using the same formula, with the point $P$ replaced by the unit $u_{1}:=-w+1$, which is the root of $x^{4}-4 x^{3}+9 x^{2}-3 x+1=0$ in $M$, and its Galois conjugates $u_{2}, u_{3}$, and $u_{4}$.

It then turns out that, to 20 digits of 13-adic accuracy,

$$
\int_{\gamma_{g_{\alpha}}} f_{a} \cdot g=-\zeta_{3}^{2} \cdot \mathcal{E}_{\alpha} \cdot \frac{\log _{E_{a}, 13}\left(P_{\mathbb{Q}}^{(a)}\right) \log _{E_{a}, 13}\left(P_{g_{\alpha}}^{(a)}\right)}{\log _{13}\left(u_{g_{\alpha}}\right)},
$$




$$
\int_{\gamma_{\beta}} f_{a} \cdot g=-\zeta_{3} \cdot \mathcal{E}_{\beta} \cdot \frac{\log _{E_{a}, 13}\left(P_{\mathbb{Q}}^{(a)}\right) \log _{E_{a}, 13}\left(P_{g_{\beta}}^{(a)}\right)}{\log _{13}\left(u_{g_{\beta}}\right)},
$$

where $\mathcal{E}_{\alpha}$ is defined as in (79), and likewise for $\mathcal{E}_{\beta}$.

A similar pattern recurs with the elliptic curve $E_{b}$, for which it was checked to 20 digits of 13 -adic precision that

$$
\begin{aligned}
& \int_{\gamma_{g_{\alpha}}} f_{b} \cdot h=\frac{\zeta}{3} \cdot \mathcal{E}_{\alpha} \cdot \frac{\log _{E_{b}, 13}\left(P_{\mathbb{Q}}^{(b)}\right) \log _{E_{b}, 13}\left(P_{g_{\alpha}}^{(b)}\right)}{\log _{13}\left(u_{g_{\alpha}}\right)}, \\
& \int_{\gamma_{g_{\beta}}} f_{b} \cdot h=-\frac{\zeta^{-1}}{3} \cdot \mathcal{E}_{\beta} \cdot \frac{\log _{E_{b}, 13}\left(P_{\mathbb{Q}}^{(b)}\right) \log _{E_{b}, 13}\left(P_{g_{\beta}}^{(b)}\right)}{\log _{13}\left(u_{g_{\beta}}\right)},
\end{aligned}
$$

with $\mathcal{E}_{\alpha}$ as in (79), and likewise for $\mathcal{E}_{\beta}$. (Here, $\zeta \cdot \mathcal{E}_{\alpha} / 3=\zeta^{-1} \cdot \mathcal{E}_{\beta} / 3=4 /(13 \sqrt{3})$.)

The choice of the prime $p=13$ in the above calculations was dictated largely by convenience. This prime has the virtue of splitting completely in $L=\mathbb{Q}(\zeta)$, so that $L$ embeds into $\mathbb{Q}_{13}$, and it divides the level of $f_{a}$ and $f_{b}$, which can thus be viewed as overconvergent 13-adic modular forms of tame level 133.

The weight-one tetrahedral form $g$ of level 133 was considered by Chinburg [Ch] in connection with Stark's original conjectures, motivated by the final sentence in [St2],

A meaningful verification for $N=133$ would be of some interest.

Stark's concluding comment follows the verification of his conjecture for the weight-one modular form considered in Example 4.1.

The next example involves the tetrahedral modular form of minimal level $124=4 \cdot 31$ [Bu, Section 2.2] which is also considered in [Ch]. It illustrates our conjecture in both rank- $(1,1)$ and rank-2 exotic settings.

EXAMPLE 5.4. Let $\chi$ be a sextic Dirichlet character of conductor $124=4 \cdot 31$, having order 2 at 4 and order 3 at 31 . Denote by $\zeta$ a primitive 12th root of unity, and define $\zeta_{3}:=\zeta^{2}-1$, a primitive cube root of unity. The space $S_{1}(124, \chi)$ is defined over $\mathbb{Q}\left(\zeta_{3}\right)$ and has dimension two. Just as in the previous example, it contains two eigenforms, which are defined over $\mathbb{Q}(\zeta)$ and conjugate over $\mathbb{Q}\left(\zeta_{3}\right)$, namely

$$
\begin{aligned}
h= & q+\zeta^{3} q^{2}+\zeta q^{3}-q^{4}-\zeta^{2} q^{5}+\left(\zeta^{2}-1\right) q^{6}-\zeta q^{7} \\
& -\zeta^{3} q^{8}+\left(-\zeta^{3}+\zeta\right) q^{10}+\left(\zeta^{3}-\zeta\right) q^{11}-\zeta q^{12}+\zeta^{2} q^{13}+\cdots
\end{aligned}
$$


and its conjugate obtained by replacing $\zeta$ by $-\zeta$. Let $g=h^{*}$. The field cut out by the projective representation attached to $g$ is the normal closure $H$ of the quartic field

$$
M=\mathbb{Q}(w), \quad w^{4}+7 w^{2}-2 w+14=0,
$$

which has discriminant $124^{2}$ and Galois group isomorphic to $A_{4}$.

Consider the elliptic curve

$$
E: y^{2}=x^{3}+x^{2}-2 x+1
$$

of conductor 124 labelled $124 a$ in Cremona's tables, and write $f$ for the associated weight-two newform. The curve $E$ has rank 1 over $\mathbb{Q}$ (with a torsion subgroup of order 3 ) and rank 2 over $M$-this places us in the rank-(1,1) setting. Following the same notation of the previous example, generators for $E(\mathbb{Q})$ and for the subgroup of points in $E(M)$ of trace zero are given by

$$
P_{\mathbb{Q}}=(-2,1), \quad P=\left(\frac{w^{3}-w^{2}+3 w-7}{2}, \frac{w^{3}+4 w^{2}+9 w+10}{4}\right) .
$$

These points were found using a naive search.

Choosing the prime $p=13$ as in the previous example, the Hecke polynomial $t^{2}-a_{13}(h) t+\chi(13)$ has roots $\beta_{h}=1$ and $\alpha_{h}=\zeta_{3}$. Hence $\alpha_{g}=1$ and $\beta_{g}=\zeta_{3}^{2}$, and therefore the 13-stabilizations $g_{\alpha}$ and $g_{\beta}$ of $g$ are distinct.

Choose the embedding of $\mathbb{Q}(\zeta)$ into $\mathbb{Q}_{13}$ which sends $\zeta$ to the unique 12th root of unity in $\mathbb{Z}_{13}$ which is congruent to 7 modulo 13 . The values of the 13adic iterated integrals were computed to be

$$
\begin{aligned}
& \int_{\gamma_{g_{\alpha}}} f \cdot h=684158979097768450057 \times 13 \bmod 13^{20} \\
& \int_{\gamma_{g_{\beta}}} f \cdot h=5953034774844704381281 \bmod 13^{20} .
\end{aligned}
$$

Note that $\alpha_{g} / \beta_{g}$ and $\beta_{g} / \alpha_{g}$ are both primitive cube roots of unity, and hence, just as in the previous example, the setting is the one discussed in Case 3 in the analysis in Section 5.1.1. Just as before, the points $P_{g_{\alpha}}$ and $P_{g_{\beta}}$ in $E(H)_{L}^{\mathrm{Ad}_{g}}$ can be defined as suitable linear combinations of the point $P$ and its Galois conjugates, following the formula in (76). The units $u_{g_{\alpha}}$ and $u_{g_{\beta}}$ are likewise constructed using the same formula, with the point $P$ replaced by the unit $u_{1}:=w^{2}-w+3$, the unique root of $x^{4}+2 x^{3}+18 x^{2}-6 x+1$ in $M$.

With these conventions in place, it has been checked to 20 digits of 13-adic precision that

$$
\int_{\gamma_{g \alpha}} f \cdot h=\frac{2}{\sqrt{3}} \cdot \mathcal{E}_{\alpha} \cdot \frac{\log _{E, 13}\left(P_{\mathbb{Q}}\right) \log _{E, 13}\left(P_{g_{\alpha}}\right)}{\log _{13}\left(u_{g_{\alpha}}\right)},
$$




$$
\int_{\gamma_{g_{\beta}}} f \cdot h=\frac{2}{\sqrt{3}} \cdot \mathcal{E}_{\beta} \cdot \frac{\log _{E, 13}\left(P_{\mathbb{Q}}\right) \log _{E, 13}\left(P_{g_{\beta}}\right)}{\log _{13}\left(u_{g_{\beta}}\right)},
$$

where $\mathcal{E}_{\alpha}$ is as in (79), and likewise for $\mathcal{E}_{\beta}$, with $\sqrt{3}=\zeta^{3}-2 \zeta$.

We now turn to the curve

$$
E: y^{2}+x y+y=x^{3}-x^{2}-3 x+3
$$

of conductor 26 labelled 26b. One checks

$$
r(E)=0, r\left(E, \operatorname{Ad}_{g}\right)=2,
$$

and likewise for analytic ranks, and so here we are in the rank-2 setting. The required points in $E(H)^{\operatorname{Ad}_{g}}$ may be constructed from the generators

$$
\begin{aligned}
P= & \frac{283 w^{3}+1295 w^{2}+1442 w+9528}{4802}, \\
& \left.\frac{29371 w^{3}-35875 w^{2}+146552 w-319814}{235298}\right) \\
Q= & \frac{-25 w^{3}+98 w^{2}+41 w+775}{121}, \\
& \left.\frac{67 w^{3}+3832 w^{2}+2465 w+17404}{1331}\right)
\end{aligned}
$$

of the rank-2 Mordell-Weil lattice in $E(M)$. We define $P_{1}$ and $P_{g_{\alpha}}$ as in the Case 3 analysis for tetrahedral forms (and similarly $P_{g_{\beta}}$ switching $\zeta_{3}$ for $\zeta_{3}^{-1}$ ). Likewise we define the points $Q_{1}, Q_{g_{\alpha}}$, and $Q_{g_{\beta}}$.

Writing now $f$ for the form attached to $E$ we find, with the same embedding as before, that

$$
\begin{gathered}
\int_{\gamma_{g \alpha}} f \cdot h=6721481652857547558786 \bmod 13^{20} \\
\int_{\gamma_{g \beta}} f \cdot h=-2549577176522983012568 \bmod 13^{20} .
\end{gathered}
$$

Then, to 20 digits of 13 -adic precision,

$$
\begin{aligned}
& \int_{\gamma_{g_{\alpha}}} f \cdot h=\frac{\zeta_{3} \cdot \mathcal{E}_{\alpha}}{\sqrt{3}} \cdot \frac{\log _{E, 13}\left(P_{1}\right) \log _{E, 13}\left(Q_{g_{\alpha}}\right)-\log _{E, 13}\left(Q_{1}\right) \log _{E, 13}\left(P_{g_{\alpha}}\right)}{\log _{p} u_{g_{\alpha}}}(81) \\
& \int_{\gamma_{g_{\beta}}} f \cdot h=-\frac{\zeta_{3}^{-1} \cdot \mathcal{E}_{\beta}}{\sqrt{3}} \cdot \frac{\log _{E, 13}\left(P_{1}\right) \log _{E, 13}\left(Q_{g_{\beta}}\right)-\log _{E, 13}\left(Q_{1}\right) \log _{E, 13}\left(P_{g_{\beta}}\right)}{\log _{p} u_{g_{\beta}}},
\end{aligned}
$$

where $\mathcal{E}_{\alpha}$ is as in (79), and likewise for $\mathcal{E}_{\beta}$, exactly as predicted by Conjecture ES. 
Finally, we consider the curve

$$
E: y^{2}=x^{3}+x-10
$$

of conductor 52 labelled $52 b$, for which again (80) holds, and so we are in the rank-2 setting. Here, we take

$$
\begin{aligned}
P= & \frac{-225 w^{3}-1533 w^{2}-1458 w+1078}{2809}, \\
& \left.\frac{-247041 w^{3}+68295 w^{2}-1265094 w+317530}{297754}\right), \\
Q= & \frac{-63391 w^{3}-51546 w^{2}-698338 w-942346}{390625}, \\
& \left.\frac{84293978 w^{3}-33352782 w^{2}+603648754 w-1348508882}{244140625}\right),
\end{aligned}
$$

as generators for the Mordell-Weil lattice in $E(M)$, and construct the points $P_{1}$, $P_{g_{\alpha}}, P_{g_{\beta}}$ and $Q_{1}, Q_{g_{\alpha}}, Q_{g_{\beta}}$ from them. Letting $f$ now be the newform attached to $E$, we have

$$
\begin{aligned}
& \int_{\gamma_{g_{\alpha}}} f \cdot h=-1602512412162131808818 \bmod 13^{20} \\
& \int_{\gamma_{g_{\beta}}} f \cdot h=4989460414989865668627 \bmod 13^{20}
\end{aligned}
$$

and one checks to 20 digits of 13-adic precision that again (81) and (82) hold. (The algebraic constants which occur in equation (81) for $26 b$ and $52 a$ are in fact exactly the same, and likewise for (82): both curves have nonsplit multiplicative reduction at 13.)

The following example illustrates the vanishing of $p$-adic iterated integrals predicted by Conjecture ES in a rank- 2 setting but where the regulator term vanishes for trivial reasons.

EXAMPLE 5.5. Consider the curve

$$
E: y^{2}+x y=x^{3}-18 x+31
$$

of conductor $4811=17 \cdot 283$ labelled $4811 a$ in Cremona's table, and write $f$ for the associated weight- 2 newform. Letting $h$ be the octahedral weight-one form in level 283 with quadratic character and $g=h^{*}$, it is readily checked that

$$
r(E / \mathbb{Q})=r_{\text {an }}(E / \mathbb{Q})=2, \quad r_{\text {an }}\left(E, \operatorname{Ad}_{g}\right)=0 .
$$


(The third equality was obtained by calculating $L\left(E, \operatorname{Ad}_{g}, 1\right)$ numerically to a modest precision sufficient to verify its nonvanishing.) Taking $p$ a regular prime for $\rho_{g}$, it is clear that Hypotheses A-C are satisfied, but that the matrix $R_{g_{\alpha}}\left(E, \varrho_{g h}\right)$ of Conjecture ES has determinant 0 . The prediction that all associated $p$-adic iterated integrals should vanish was verified for $p=17$ to 5 digits of 17-adic accuracy.

We note also here that we tested the vanishing prediction of Conjecture ES in several examples when $r\left(E, \varrho_{g h}\right)=4$ and 6 (involving CM rather than exotic forms).

Our final exotic example involves the exotic octahedral modular form of minimal level $148=4 \cdot 37$ [Bu, Section 2.3] and rank-(1,1) settings of Conjecture ES.

EXAMPLE 5.6. Let $\chi$ be the Dirichlet character of level $148=2^{2} \cdot 37$ which is trivial at 2 and has order 4 at 37 (there are two such characters and they are Galois conjugate). The space $S_{1}(148, \chi)$ is defined over $L=\mathbb{Q}(i)$ and has dimension one. It contains the single eigenform

$$
h=q-i q^{3}-q^{7}+i q^{11}+(i-1) q^{17}+(-i+1) q^{19}+\cdots .
$$

The field $H$ cut out by the projective representation attached to $g=h^{*}$ is the normal closure of the quartic field

$$
M:=\mathbb{Q}(a), \quad a^{4}-a^{3}+5 a^{2}-7 a+12
$$

of discriminant $2^{2} \cdot 37^{3}$ and having Galois group $S_{4}$. The unique quadratic subfield of $H$ is $K=\mathbb{Q}(\sqrt{37})$, and hence $\tilde{M}=M K$ is the unique quadratic extension of $M$ contained in $H$.

Testing Conjecture ES in the rank- $(1,1)$ setting requires elliptic curves $E$ of rank 1 over $\mathbb{Q}$ for which in addition $r\left(E, \operatorname{Ad}_{g}\right)=1$. We choose $p=17$ and consider elliptic curves $E$ of rank 1 and the conductor dividing $148 p$ which satisfy the necessary hypotheses. (Restricting the conductor of $E$ in this way makes it possible to confine the 17 -adic iterated integral computations to spaces of overconvergent $p$-adic modular forms tame level 148.) Analytic rank computations suggest these are the curves $37 a, 629 a, 629 c$, and $629 d$ in Cremona's tables.

Following the general notation introduced in Section 5.1.2, Conjecture ES was verified for each of $E=37 a, 629 a$, and $629 d$ using points $P \in E(\tilde{M})^{-}$found by Steve Donnelly. (For $629 c$, the authors were unable to find the point $P$. This leaves opens possibility of constructing it using Conjecture ES, a challenge that has not yet been addressed.) 
The elliptic curves of interest are

$$
\begin{gathered}
E_{37 a}: y^{2}+y=x^{3}-x \\
E_{629 a}: y^{2}+x y=x^{3}-x^{2}+11 x-18 \\
E_{629 d}: y^{2}+x y+y=x^{3}-x^{2}-171 x+1904
\end{gathered}
$$

with associated modular forms $f_{37 a}, f_{629 a}$, and $f_{629 d}$.

With $p=17$, the Hecke eigenvalues for the octahedral form $h$ are $\beta_{h}=i$ and $\alpha_{h}=-1$, and therefore $\alpha_{g}=-i$ and $\beta_{g}=-1$. It follows that the Frobenius eigenvalues at 17 for the adjoint representation $\operatorname{Ad}_{g}$ are $1, \alpha / \beta=i$, and $\beta / \alpha=-i$, which places this experiment in Case 5 of the analysis in Section 5.1.2.

Choose the embedding of $L=\mathbb{Q}(i)$ into $\mathbb{Q}_{17}$ which sends $i$ to the unique square root of -1 which is congruent to 4 modulo 17 . A calculation of iterated integrals to 15 digits of 17 -adic precision reveals that

$$
\begin{aligned}
& \int_{\gamma_{g \alpha}} f_{37 a} \cdot h=795182232610993468 \bmod 17^{15} \\
& \int_{\gamma_{g_{\beta}}} f_{37 a} \cdot h=591309544450892129 \bmod 17^{15} \\
& \int_{\gamma_{g \alpha}} f_{629 a} \cdot h=231921888434186915 \bmod 17^{15} \\
& \int_{\gamma_{\beta \beta}} f_{629 a} \cdot h=776162921415439615 \bmod 17^{15} \\
& \int_{\gamma_{g \alpha}} f_{629 d} \cdot h=-612176557243786922 \times 17 \bmod 17^{15} \\
& \int_{\gamma_{\beta \beta}} f_{629 d} \cdot h=329220710071055453 \bmod 17^{15} .
\end{aligned}
$$

Consider first the elliptic curve $E=E_{37 a}$. The generator of $E(\mathbb{Q})$ is chosen to be $P_{\mathbb{Q}}=(0,0)$. The point

$$
\begin{aligned}
& \left(\frac{-152551 a^{3}+327117 a^{2}-384541 a+1037221}{21316},\right. \\
& \left.\frac{-110958819 a^{3}+69702635 a^{2}+95157821 a+314525497}{3112136}\right)
\end{aligned}
$$


on the quadratic twist $y^{2}+y=x^{3}-1369 x+12663$ of $E$ by 37 gives rise to the point

$$
\begin{aligned}
P= & \left(\frac{-10817 b^{7}-15535 b^{6}-11412 b^{5}-43863 b^{4}-97162 b^{3}+30857 b^{2}+39063 b-42}{21316},\right. \\
& \left.\frac{2553946 b^{7}-379967 b^{6}-1189613 b^{5}+10482748 b^{4}+12706439 b^{3}-35039416 b^{2}+19176309 b+6012477}{15560680}\right)
\end{aligned}
$$

in $E(\tilde{M})^{-}$, where

$$
\tilde{M}=\mathbb{Q}(b), \quad b^{8}+b^{7}+b^{6}+4 b^{5}+8 b^{4}-4 b^{3}+b^{2}-b+1=0 .
$$

Writing $P_{1}, P_{2}, P_{3}$, and $P_{4}$ for the Galois translates of $P$ so that they are permuted cyclically by $\sigma_{17}$, define

$$
P_{g_{\alpha}}=P_{1}-i P_{2}-P_{3}+i P_{4}, \quad P_{g_{\beta}}=P_{1}+i P_{2}-P_{3}-i P_{4},
$$

following (78) and (77). Likewise, taking $u:=-b^{2}$ as one of the two roots of $x^{8}-x^{7}+9 x^{6}-10 x^{5}+102 x^{4}-10 x^{3}+9 x^{2}-x+1$ in $\tilde{M}$ leads to a definition of the Stark units $u_{g_{\alpha}}$ and $u_{g_{\beta}} \in\left(\mathcal{O}_{H}^{\times}\right)_{L}^{\operatorname{Ad}_{g}}$ attached to the two stabilizations of $g$.

The following identities were verified to 15 digits of 17 -adic precision:

$$
\begin{aligned}
& \int_{\gamma_{g_{\alpha}}} f_{37 a} \cdot h=\frac{4}{3} \cdot \mathcal{E}_{\alpha} \cdot \frac{\log _{E, 17}\left(P_{\mathbb{Q}}\right) \log _{E, 17}\left(P_{g_{\alpha}}\right)}{\log _{17}\left(u_{g_{\alpha}}\right)}, \\
& \int_{\gamma_{g_{\beta}}} f_{37 a} \cdot h=\frac{4}{3} \cdot \mathcal{E}_{\beta} \cdot \frac{\log _{E, 17}\left(P_{\mathbb{Q}}\right) \log _{E, 17}\left(P_{g_{\beta}}\right)}{\log _{17}\left(u_{g_{\beta}}\right)},
\end{aligned}
$$

with $\mathcal{E}_{\alpha}$ as in (79), and likewise for $\mathcal{E}_{\beta}$.

Consider next the elliptic curve $E=E_{629 a}$. Here, the generator for $E(\mathbb{Q})$ is chosen to be $P_{\mathbb{Q}}=(2,2)$, and, just as above, the point

$$
\begin{aligned}
P=( & \frac{-5 b^{7}-11 b^{6}-16 b^{5}-31 b^{4}-74 b^{3}-47 b^{2}-25 b+57}{36}, \\
& \left.\frac{1083 b^{7}+1784 b^{6}+2366 b^{5}+5569 b^{4}+11572 b^{3}+3647 b^{2}+1907 b-3464}{1080}\right)
\end{aligned}
$$

belongs to $E(\tilde{M})^{-}$. The points $P_{g_{\alpha}}$ and $P_{g_{\beta}}$, and the units $u_{g_{\alpha}}$ and $u_{g_{\beta}}$, are defined just as they were before. It was then checked to 15 digits of 17 -adic precision that

$$
\begin{aligned}
& \int_{\gamma_{\gamma_{\alpha}}} f_{629 a} \cdot h=\frac{-2 i-2}{3} \cdot \mathcal{E}_{\alpha} \cdot \frac{\log _{E, 17}\left(P_{\mathbb{Q}}\right) \log _{E, 17}\left(P_{g_{\alpha}}\right)}{\log _{17}\left(u_{g_{\alpha}}\right)}, \\
& \int_{\gamma_{g_{\beta}}} f_{629 a} \cdot h=\frac{2 i-2}{3} \cdot \mathcal{E}_{\beta} \cdot \frac{\log _{E, 17}\left(P_{\mathbb{Q}}\right) \log _{E, 17}\left(P_{g_{\beta}}\right)}{\log _{17}\left(u_{g_{\beta}}\right)},
\end{aligned}
$$

with $\mathcal{E}_{\alpha}$ as in (79), and likewise for $\mathcal{E}_{\beta}$. 
Finally, for the curve $E=E_{629 d}$, let $P_{\mathbb{Q}}=(16,47)$ be the Mordell-Weil generator for $E(\mathbb{Q})$, and let

$$
\begin{aligned}
P=( & \frac{-1369 b^{7}+259 b^{6}-518 b^{5}-4625 b^{4}-5994 b^{3}+12987 b^{2}-17871 b+9656}{400}, \\
& \left.\frac{-1258148 b^{7}+64491 b^{6}-649461 b^{5}-5104594 b^{4}-5377617 b^{3}+14354298 b^{2}-11310937 b+2516289}{40000}\right)
\end{aligned}
$$

be the generator of $E(\tilde{M})_{L}^{-}$.

With the same definitions for the points $P_{g_{\alpha}}$ and $P_{g_{\beta}}$ in terms of $P$ as before, the following remarkably simple relation was checked to 15 digits of 17 -adic precision:

$$
\begin{aligned}
& \int_{\gamma_{g \alpha}} f_{629 d} \cdot h=(-10 i+10) \cdot \mathcal{E}_{\alpha} \cdot \frac{\log _{E, 17}\left(P_{\mathbb{Q}}\right) \log _{E, 17}\left(P_{g_{\alpha}}\right)}{\log _{17}\left(u_{g_{\alpha}}\right)}, \\
& \int_{\gamma_{g_{\beta}}} f_{629 d} \cdot h=(-10 i-10) \cdot \mathcal{E}_{\beta} \cdot \frac{\log _{E, 17}\left(P_{\mathbb{Q}}\right) \log _{E, 17}\left(P_{g_{\beta}}\right)}{\log _{17}\left(u_{g_{\beta}}\right)},
\end{aligned}
$$

with $\mathcal{E}_{\alpha}$ as in (79), and likewise for $\mathcal{E}_{\beta}$. Here, $(-10 i+10) \cdot \mathcal{E}_{\alpha}=160 / 17$ and $(-10 i-10) \cdot \mathcal{E}_{\beta}=-160 / 17$.

\section{Stark points attached to odd two-dimensional Artin representations}

This section considers the case where $g \in S_{1}(N, \bar{\chi})$ is a cuspidal newform and $h=E(1, \chi)$ is the Eisenstein series attached to the pair $(1, \chi)$. In that case,

$$
V_{g h}=V_{g} \oplus V_{g^{*}}, \quad L\left(E, \varrho_{g h}, s\right)=L\left(E, \varrho_{g}, s\right) L\left(E, \bar{\varrho}_{g}, s\right) .
$$

The factorization of $L$-functions guarantees that Hypothesis A of the introduction is automatically satisfied in this setting, but Hypothesis B is only satisfied when $L\left(E, \varrho_{g}, 1\right)=0$. Furthermore, the conditions formulated in Conjecture ES are fulfilled precisely when $L\left(E, \varrho_{g h}, s\right)$ has a double zero at $s=1$, so that

$$
r_{\mathrm{an}}\left(E, V_{g}\right)=r_{\mathrm{an}}\left(E, \bar{V}_{g}\right)=1 .
$$

The eigenvalues of $\sigma_{p}$ acting on $V_{g}$ and on $V_{h}$, respectively, are given by

$$
\alpha_{g}, \quad \beta_{g}, \quad \alpha_{h}=1, \quad \beta_{h}=\chi(p)=\left(\alpha_{g} \beta_{g}\right)^{-1},
$$

and therefore

$$
\alpha_{g} \alpha_{h}=\alpha_{g}, \quad \alpha_{g} \beta_{h}=\bar{\beta}_{g}
$$


Let $H$ be the Galois extension of $\mathbb{Q}$ through which $\varrho_{g}$ (or, equivalently, $\bar{\varrho}_{g}$ ) factors. Assuming that $V_{g}$ is not isomorphic to its contragredient, its underlying field $L$ of coefficients is a CM subfield of a cyclotomic field, and the spaces $E(H)_{L}^{V_{g}}$ and $E(H)_{L}^{V_{g^{*}}}$ decompose as $\sigma_{p}$-modules as

$$
E(H)_{L}^{V_{g}}=L \cdot P_{g, \alpha} \oplus L \cdot P_{g, \beta}, \quad E(H)_{L}^{\bar{V}_{g}}=L \cdot P_{g^{*}, \bar{\alpha}} \oplus L \cdot P_{g^{*}, \bar{\beta}},
$$

where $P_{g, \alpha}$ is an eigenvector for $\sigma_{p}$ with eigenvalue $\alpha_{g}$, and similar conventions are adopted for the other points.

When $g$ is regular at $p$ and (14) is satisfied-and so Hypothesis C holds by Proposition 1.1-Conjecture ES of the introduction predicts that, for a suitable choice of test vectors,

$$
\left(\breve{f}, \breve{\gamma}_{g_{\alpha}}, h\right) \in S_{2}(N)_{L} \times S_{1}(N, \chi)_{L}^{\vee}\left[g_{\alpha}\right] \times M_{1}(N, \chi)_{L}
$$

(and likewise with $\alpha_{g}$ replaced by $\beta_{g}$ ), the following identities hold:

$$
\begin{aligned}
\int_{\breve{\gamma}_{g \alpha}} \breve{f} \cdot \breve{h} & =\frac{\log _{E, p}\left(P_{g, \alpha}\right) \cdot \log _{E, p}\left(P_{g^{*}, \bar{\beta}}\right)}{\log _{p}\left(u_{g_{\alpha}}\right)}, \\
\int_{\breve{\gamma}_{g_{\beta}}} \breve{f} \cdot \breve{h} & =\frac{\log _{E, p}\left(P_{g, \beta}\right) \cdot \log _{E, p}\left(P_{g^{*}, \bar{\alpha}}\right)}{\log _{p}\left(u_{g_{\beta}}\right)},
\end{aligned}
$$

where $u_{g_{\alpha}}$ is precisely the same Stark unit as arose in Section 5.

The identities above turned out to be harder to check experimentally, for the following reasons.

- Instances where $L\left(E, \varrho_{g}, s\right)$ acquires a simple zero at $s=1$ are ostensibly sporadic in nature. When $\varrho_{g}$ is not isomorphic to its contragredient representation, this $L$-function does not admit a sign in its functional equation which might be used to systematically predict the vanishing of $L\left(E, \varrho_{g}, 1\right)$, and it is expected that cases where $L\left(E, \varrho_{g}, 1\right)=0$ are exceedingly rare (although very little data has been gathered to be able to make serious pronouncements in this direction).

- The field cut out by $\varrho_{g}$ is larger than the field cut out by its adjoint $\operatorname{Ad}_{g}$. More precisely, it is an abelian extension of it, of degree $t \geqslant 2$, where $t$ is the order of the nontrivial central character attached to $\varrho_{g}$. In particular, the point $P_{g, \alpha}$ that arises in this section is entirely independent of the point denoted $P_{g_{\alpha}}$ in Section 5, is defined over a larger degree extension, and is therefore harder to produce numerically in practice, in addition to not arising in as systematic a manner. 
The problem of producing numerical evidence becomes more tractable when $g=g^{*}$, which occurs precisely when $g$ is induced from a ring class character of an imaginary quadratic field. Such examples are arguably less interesting, since they also fall under the scope of Section 3. See the second half of Example 3.12 of Section 3.3 for a numerical verification of Conjecture ES in the case where $g$ is further assumed to be self-dual. (See [DLR1, Example 5.2] though for numerical evidence when $g \neq g^{*}$ in the analogous setting in which $f$ is replaced by a weight-two Eisenstein series, and Mordell-Weil groups are replaced by unit groups.)

\section{Stark points over cyclotomic fields and Eisenstein series}

7.1. Theoretical preliminaries. This section turns to the case where $g$ is an Eisenstein series satisfying condition (2) of Hypothesis $\mathrm{C}^{\prime}$. Recall that, in this case, $V_{g h}^{g \alpha}:=W \otimes V_{h}$, where $W$ is any line in $V_{g}$ which is not an eigenspace for the global Galois action, that is, not one of the two one-dimensional irreducible summands of $V_{g}$. Conjecture ES of the introduction predicts that, for a suitable choice of test vectors

$$
\left(\breve{f}, \breve{\gamma}_{g_{\alpha}}, \breve{h}\right) \in S_{2}(N)_{L} \times S_{1}(N, \chi)_{L}^{\vee}\left[g_{\alpha}\right] \times M_{1}(N, \chi)_{L}
$$

(and likewise with $\alpha_{g}$ replaced by $\beta_{g}$ ), the following identities hold:

$$
\begin{aligned}
\int_{\breve{\gamma}_{g \alpha}} \breve{f} \cdot \breve{h} & =\frac{\log _{E, p}\left(P_{g, \alpha}\right) \cdot \log _{E, p}\left(P_{g^{*}, \bar{\beta}}\right)}{\log _{p}\left(u_{g_{\alpha}}\right)}, \\
\int_{\breve{\gamma}_{g_{\beta}}} \breve{f} \cdot \breve{h} & =\frac{\log _{E, p}\left(P_{g, \beta}\right) \cdot \log _{E, p}\left(P_{g^{*}, \bar{\alpha}}\right)}{\log _{p}\left(u_{g_{\beta}}\right)},
\end{aligned}
$$

where $u_{g_{\alpha}}$ and $u_{g_{\beta}}$ are the Gross-Stark $p$-units rather than genuine units. Otherwise, the statement of Conjecture ES is identical to the case (83) where the roles of $g$ and $h$ are interchanged.

For the same reasons as were discussed in the previous section, we have been unable to gather much experimental evidence for it, except in the simplest (and particularly appealing) special case where $g$ and $h$ are both weight-one Eisenstein series of the form

$$
g=E_{1}\left(1, \chi^{-1}\right), \quad h=E_{1}\left(\chi_{1}, \chi_{2}\right) \quad \text { where } \chi_{1} \chi_{2}=\chi .
$$

It is natural to restrict attention to the case where at least one of $\chi_{1}$ or $\chi_{2}$ is not quadratic, to exclude scenarios that were already treated in Section 3, where both $V_{g}$ and $V_{h}$ are induced from genus characters of the imaginary quadratic field 
attached to the quadratic Dirichlet character $\chi$. Let $L$ be the field in which the characters $\chi_{1}$ and $\chi_{2}$ take values. The representations $V_{g}, V_{h}$, and $V_{g h}$ decompose as direct sums of one-dimensional representations:

$$
V_{g}=L \oplus L\left(\chi^{-1}\right), \quad V_{h}=L\left(\chi_{1}\right) \oplus L\left(\chi_{2}\right), \quad V_{g h}=L\left(\chi_{1}\right) \oplus L\left(\chi_{2}\right) \oplus L\left(\bar{\chi}_{1}\right) \oplus L\left(\bar{\chi}_{2}\right) .
$$

It is worth noting that the representation $V_{g h}$ is isomorphic to its dual, but that its irreducible constituents are not all self-dual when $\chi_{1}$ or $\chi_{2}$ is nonquadratic. This phenomenon, which is specific to the case where at least one of the forms $g$ or $h$ is an Eisenstein series, makes the occurrence of situations where $L$-functions vanish somewhat more rare and sporadic, just as in the setting described in Section 6.

The adjoint representation $\operatorname{Ad}_{g}$ attached to $V_{g}$ breaks up as a direct sum

$$
\operatorname{Ad}_{g}=L \oplus L(\chi) \oplus L\left(\chi^{-1}\right) \text {. }
$$

Let $H_{\chi}$ be the abelian extension of $\mathbb{Q}$ cut out by the character $\chi$. Recall that Hypothesis $\mathrm{C}^{\prime}$ requires that

$$
\chi(p)=1 \text {; that is, } V_{g} \text { is not regular and } \chi_{1}(p)=\chi_{2}(p)^{-1} .
$$

Since $\chi$ is nonquadratic, the $L$-vector spaces

$$
\left(\mathcal{O}_{H_{\chi}}[1 / p]^{\times}\right)_{L}^{\chi}, \quad\left(\mathcal{O}_{H_{\chi}}[1 / p]^{\times}\right)_{L}^{\chi^{-1}}, \quad\left(\mathcal{O}_{H_{\chi}}[1 / p]^{\times}\right)_{L}^{\chi+\chi^{-1}}
$$

have dimensions one, one, and two, respectively. Let $\left.u_{g_{\alpha}} \in \mathcal{O}_{H_{\chi}}[1 / p]^{\times}\right)_{L}^{\chi+\chi^{-1}}$ be the Gross-Stark unit associated to $g_{\alpha}$ following the recipe of Section 1.2.

Let $f$ be a weight-two cusp form attached to an elliptic curve. Note that Hypothesis $\mathrm{A}$ is automatically satisfied in this setting. When $r_{\text {an }}\left(E, \chi_{1} \oplus \chi_{2}\right)>0$ (and so Hypothesis B holds), Conjecture ES predicts that the iterated integrals attached to $(f, g, h)$ is nonvanishing if and only if $r_{\mathrm{an}}\left(E, \chi_{1} \oplus \chi_{2}\right)=1$. Assume (after possibly reordering $\chi_{1}$ and $\chi_{2}$ ) that

$$
r_{\mathrm{an}}\left(E, \chi_{1}\right)=1, \quad r_{\mathrm{an}}\left(E, \chi_{2}\right)=0 .
$$

Under this assumption, Conjecture ES predicts the existence of points $P_{\chi_{1}} \in$ $E\left(H_{\chi_{1}}\right)_{L}^{\chi_{1}}$ and $P_{\bar{\chi}_{1}} \in E\left(H_{\chi_{1}}\right)_{L}^{\bar{\chi}_{1}}$ for which

$$
\int_{\gamma} f \cdot h \stackrel{?}{=} \frac{\log _{E, p}\left(P_{\chi_{1}}\right) \log _{E, p}\left(P_{\bar{\chi}_{1}}\right)}{\log _{p}\left(u_{g_{\alpha}}\right)} .
$$


A notable feature of this conjecture is that it involves global points over abelian extensions of $\mathbb{Q}$, whose occurrence seems sporadic, in contrast with the self-dual setting that is germane to the theory of Heegner and Stark-Heegner points.

7.2. Numerical evidence. Although Eisenstein series are plentiful, it is the very scarcity of points over odd-degree abelian extensions, and the necessity of working at a prime $p$ which splits completely in this abelian extension, which make the numerical verification of Conjecture ES so challenging in this situation. Frequently, when such a point does exist, the common level of the Eisenstein series and cusp form $f$, and size of the prime $p$, push the example beyond the current reach of experimentation.

The authors were rather fortunate to encounter the following two examples (and in addition another cubic field example in which $r_{\text {an }}\left(E, \chi_{1} \oplus \chi_{2}\right)=2$ and the integral vanished, as predicted by Conjecture ES). In the first one, we give for variety numerical details of the logarithms computed-these were suppressed elsewhere to lighten the burden of unilluminating long numbers in the paper.

EXAMPLE 7.1. Let

$$
E: y^{2}+x y+y=x^{3}+x^{2}-4 x+5
$$

be the elliptic curve of conductor $2 \cdot 3 \cdot 7$ denoted $42 a$ in the tables of Cremona. Let $\chi_{1}$ be the (even) cubic character of conductor 19, and let $\chi_{2}$ be the (odd) quadratic character of conductor 3 , so that $\chi=\chi_{1} \chi_{2}$ is an odd sextic character of conductor 57. As before, write $L=\mathbb{Q}\left(\zeta_{3}\right)$ for the field of coefficients generated by the values of $\chi_{1}$. A direct calculation reveals that

$$
E(\mathbb{Q})_{L}=0, \quad r\left(E, \chi_{1}\right)=r\left(E, \bar{\chi}_{1}\right)=1, \quad r\left(E, \chi_{2}\right)=r\left(E, \bar{\chi}_{2}\right)=0,
$$

and likewise for the analytic ranks. More precisely, the cyclic cubic field cut out by $\chi_{1}$ is given concretely by

$$
H_{\chi_{1}}=\mathbb{Q}(\alpha) \quad \text { where } \alpha \text { satisfies } \alpha^{3}-\alpha^{2}-6 \alpha+7=0 .
$$

The Mordell-Weil group $E\left(H_{\chi_{1}}\right)$ has rank 2, and is generated as a $\operatorname{Gal}\left(H_{\chi_{1}} / \mathbb{Q}\right)$ module by the point

$$
P:=\left(2 \alpha^{2}+3 \alpha-8,4 \alpha^{2}+6 \alpha-10\right)
$$

with coordinates in $H_{\chi_{1}}$. 
Fix the prime $p=7$, and let $\zeta_{3}$ be the unique cube root of unity in $\mathbb{Q}_{7}$ which is congruent to 2 modulo 7 . Fix a primitive 6 th root of unity $\zeta_{6}=-\bar{\zeta}_{3}$, so $\zeta_{3}=\zeta_{6}^{2}$. After letting $\sigma$ be a generator of the Galois group $\operatorname{Gal}\left(H_{\chi_{1}} / \mathbb{Q}\right)$, let

$$
\begin{aligned}
& P_{\chi_{1}}=P+\bar{\chi}_{1}(\sigma) P^{\sigma}+\bar{\chi}_{1}\left(\sigma^{2}\right) P^{\sigma^{2}}, \\
& P_{\bar{\chi}_{1}}=P+\chi_{1}(\sigma) P^{\sigma}+\chi_{1}\left(\sigma^{2}\right) P^{\sigma^{2}} .
\end{aligned}
$$

A numerical evaluation of the $p$-adic logarithms shows that

$$
\begin{aligned}
& \log _{E, 7}\left(P_{\chi_{1}}\right)=-199213220217977173722637405653124 \cdot 7 \bmod 7^{38} \\
& \log _{E, 7}\left(P_{\bar{\chi}_{1}}\right)=-61436332416679587407622380051451 \cdot 7^{2} \bmod 7^{38} .
\end{aligned}
$$

Turning now to the Gross-Stark unit attached to $g$, the defining polynomial of the sextic field $H_{\chi}$ is chosen to be $x^{6}+2 x^{5}-2 x^{4}-14 x^{3}+55 x^{2}+19 x+343$, whose roots generate the group of 7-units in $H_{x}$. Let $u$ be the unique 7-adic root of this polynomial satisfying $\operatorname{ord}_{7}(u)=3$, and set

$$
\begin{aligned}
& u_{\chi}:=\sum_{\sigma \in \operatorname{Gal}\left(H_{\chi} / \mathbb{Q}\right)} \bar{\chi}(\sigma) \otimes u^{\sigma}, \\
& u_{\bar{\chi}}:=\sum_{\sigma \in \operatorname{Gal}\left(H_{\chi} / \mathbb{Q}\right)} \chi(\sigma) \otimes u^{\sigma} .
\end{aligned}
$$

An evaluation of the 7-adic logarithms of these units yields

$$
\begin{aligned}
& \log _{7}\left(u_{\chi}\right)=793452521874901120340916121448 \times 7 \bmod 7^{38}, \\
& \log _{7}\left(u_{\bar{\chi}}\right)=2536773629823487601064250408052 \times 7 \bmod 7^{38},
\end{aligned}
$$

and

$$
\log _{7}\left(u_{\chi}\right)+\log _{7}\left(u_{\bar{\chi}}\right)=475746593099769817343595218500 \times 7^{2} \bmod 7^{38} .
$$

Let

$$
g:=E_{1}\left(1, \chi^{-1}\right), \quad h:=E_{1}\left(\chi_{1}, \chi_{2}\right),
$$

and take the stabilization

$$
\breve{g}:=\left(g(q)-g\left(q^{7}\right)\right)-\left(-\zeta_{6}^{-1}+1\right)\left(g\left(q^{2}\right)-g\left(q^{14}\right)\right)
$$

in (classical) level 2.7 57 with $U_{2}$-eigenvalue 1 . These are also 7 -adic modular forms of level $114=2 \cdot 57$. Letting $\breve{\gamma}$ be the element in the dual space which sends $\breve{g}$ to 1 and the other stabilization of $E_{1}\left(1, \chi^{-1}\right)$ to zero, a computation 
of the $p$-adic iterated integral (by far the most resource intensive part of the experiment) reveals that

$$
\int_{\breve{\gamma}} f \cdot h=-1264003828062411821439581214808 \bmod 7^{36} .
$$

A direct comparison of (87) with the invariants obtained in (85) and (86) shows that

$$
\int_{\breve{\gamma}} f \cdot h=\frac{64}{7 \cdot 9} \frac{\log _{E, 7}\left(P_{\chi_{1}}\right) \log _{E, 7}\left(P_{\bar{\chi}_{1}}\right)}{\left(\log _{7}\left(u_{\chi}\right)+\log _{7}\left(u_{\bar{\chi}}\right)\right)} \bmod 7^{36} .
$$

Replacing $\breve{g}$ by the other stabilization of $E_{1}\left(1, \chi^{-1}\right)$, with $U_{2}$-eigenvalue $-\zeta_{6}^{-1}+1$, and defining $\breve{\gamma}$ accordingly, just multiplies the integral value by $\zeta_{3}^{2}$; and likewise, taking $\breve{g}$ to be a stabilization of $E_{1}\left(\chi_{1}^{-1}, \chi_{2}^{-1}\right)$ and $h:=E_{1}(1, \chi)$ in the integral changes its value by at most a cube root of unity. This is as predicted by Conjecture ES.

EXAMPLE 7.2. Consider the case where

$$
E: y^{2}+x y+y=x^{3}-5 x-8
$$

is the elliptic curve of conductor $2 \cdot 13$ denoted $26 a$ in the tables of Cremona. Write $f$ for the newform of weight two attached to $E$. Let $\eta_{1}$ be an (even) cubic character of conductor 7 , and let $\eta_{2}$ be the (odd) quadratic character of conductor 3. Define $\chi_{1}:=\eta_{1} \eta_{2}$, an odd sextic character of conductor $21, \chi_{2}:=1$, and $\chi:=\chi_{1} \chi_{2}=\chi_{1}$. (This rather roundabout notation is chosen just to align with that in Section 7.1.) Letting $g:=E_{1}\left(1, \chi^{-1}\right)$ and $h:=E_{1}\left(\chi_{1}, \chi_{2}\right)=E_{1}(\chi, 1)$ $=E_{1}(1, \chi)$, the representation $V_{g h}$ is given by

$$
V_{g h}=L \oplus L \oplus L(\chi) \oplus L(\bar{\chi}),
$$

where $L=\mathbb{Q}\left(\zeta_{3}\right)$ is the field generated by the values of $\chi$. A descent calculation reveals that

$$
E(\mathbb{Q})_{L}=0, \quad r(E, \chi)=r(E, \bar{\chi})=1,
$$

and likewise for the analytic ranks. The cyclic sextic field cut out by $\chi$ is given by

$$
H_{\chi}=\mathbb{Q}(\alpha), \quad \text { where } \alpha \text { satisfies } \alpha^{6}-\alpha^{5}+3 \alpha^{4}+5 \alpha^{2}-2 \alpha+1=0 .
$$

The Mordell-Weil group $E\left(H_{\chi}\right)$ has rank 2 , and is generated as a $\operatorname{Gal}\left(H_{\chi} / \mathbb{Q}\right)$ module by the point 


$$
\begin{aligned}
P:= & \left(2 \alpha^{5}-6 \alpha^{4}+8 \alpha^{3}-10 \alpha^{2}+4 \alpha-23,-50 \alpha^{5}\right. \\
& \left.+38 \alpha^{4}-134 \alpha^{3}-36 \alpha^{2}-242 \alpha+50\right)
\end{aligned}
$$

with coordinates in $H_{\chi}$. (This point is the image of one on the quadratic twist of $E$ by -3 over the cyclic cubic field $H_{\eta_{1}}$ cut out by $\eta_{1}$.) The points $P_{\chi}$ and $P_{\bar{\chi}}$, and the units $u_{\chi}$ and $u_{\bar{\chi}}$, are now constructed in an analogous manner to Example 7.1.

We take $p=13$. Noting here $a_{2}(g)=1+\zeta_{6}^{-1}$ with $\zeta_{6}$ a primitive sixth root of unity, we replace $g$ by its stabilization

$$
\breve{g}:=\left(g(q)-g\left(q^{13}\right)\right)-\left(g\left(q^{2}\right)-g\left(q^{26}\right)\right)
$$

in level $2 \cdot 13 \cdot 21$, and let $\breve{\gamma}$ be the element in the dual space which sends $\breve{g}$ to 1 and the other stabilization to zero. Embedding $\mathbb{Q}\left(\zeta_{6}\right)$ in $\mathbb{Q}_{13}$ so that $\zeta_{6} \equiv 4 \bmod 13$, we find

$$
\int_{\breve{\gamma}} f \cdot h=-1183909055963560023118938339892601 \times 13 \bmod 13^{30} .
$$

It was then checked to 30 digits of 13 -adic precision that

$$
\int_{\breve{\gamma}} f \cdot h=\frac{4}{13 \cdot \zeta_{6}} \frac{\log _{E, 13}\left(P_{\chi}\right) \log _{E, 13}\left(P_{\bar{\chi}}\right)}{\left(\log _{13}\left(u_{\chi}\right)+\log _{13}\left(u_{\bar{\chi}}\right)\right)},
$$

as predicted by Conjecture ES.

REMARK 7.3. The experimental work in this paper fell into two phases-one of discovery and one of verification-between which Conjecture ES was gradually formulated and refined. Formula (45) and Examples 3.11, 4.1, and 7.1 belong to the former phase, and the remaining examples to the latter. One notable feature which eluded the authors at the outset was the possibility of nonvanishing of the $p$-adic iterated integral in 'genuine rank-2' settings. This discovery was made experimentally in the companion paper [DLR1, Example 6.1], and it was this experiment which led to the definitive formulation of the conjecture.

\section{Acknowledgements}

The authors thank Kevin Buzzard for illuminating exchanges on weight-one modular forms, Mladen Dimitrov for helpful discussions around Section 1.1, Neil Dummigan for his guidance on some classical formulae relating adjoint $L$-functions and the Petersson norm, Haruzo Hida for a suggestion which helped shape the statement of the conjectures, and Mike Woodbury for sharing 
his expertise on trilinear periods. The experimental work in this paper was undertaken using the MAGMA computer algebra package, and the authors are very grateful and deeply indebted to Steve Donnelly for his help and advice on a number of computational problems.

The authors gratefully acknowledge the following institutions for their support and the pleasant locales in which their collaboration on this project was pursued: the Mathematics Center Heidelberg (MATCH), McGill's Bellairs Research Station in Barbados, the CRM and Università Politecnica de Catalunya in Barcelona, the Mathematical Institute at the University of Oxford, the Pohang Mathematics Institute in Pohang, South Korea, and the Centro de Ciencias de Benasque Pedro Pascual in the Spanish Pyrénées.

The first author was supported by an NSERC Discovery grant. The work of the middle author was supported in part by a grant from the European Research Council (204083), and largely undertaken during a sabbatical year at the University of Kyushu; he thanks Shin Hattori, Masanobu Kaneko, and Yuichiro Taguchi for their hospitality. The third author was supported by Grant MTM2012-34611.

\section{Appendix. Digest of numerical examples}

The following table summarizes some of the salient features of the numerical examples presented in support of Conjecture ES. It is intended as a navigational guide for the reader. Each numbered example involves a choice of weight-one modular forms $g$ and $h$ of some common level $N$. This level, the dimensions of the irreducible summands of the tensor product representation $V_{g h}:=V_{g} \otimes V_{h}$, and the image of each of those irreducible representations as an abstract group are given in the second, third, and fourth columns of the table, respectively. (Here, $D_{n}$ is the dihedral group of order $n$.) Each numbered example then involves a further choice of one or more elliptic curve $E$ and one or more primes $p$ with $\operatorname{gcd}(N, p)=1$. The curve $E$ always has conductor dividing $N p$ (and thus the attached form $f$ has $p$-adic level dividing $N$ ). The Cremona labels of the curves $E$ are shown in the fifth column, and the ranks with which the irreducible summands of $V_{g h}$ occur in the Mordell-Weil group of $E$ are given in the sixth. For reasons of space we do not show the different primes $p$ considered; note however that whether $p$ was chosen to divide the conductor of $E$ or not can be readily deduced by comparing columns 'Level' and 'Curves'. The examples in the table are grouped under headings indicating the nature of the points constructed. 


\begin{tabular}{cccccc}
\hline Ex. & Level & Dimension of $V_{g h}$ & Galois groups & Curves & Ranks \\
\hline \multicolumn{5}{c}{ Classical Heegner points over ring class fields of imaginary quadratic fields } \\
3.11 & 83 & $2+1+1$ & $D_{6} \times 1 \times C_{2}$ & $83 a$ & $1+1+0$ \\
3.11 & 83 & $2+2$ & $D_{6} \times D_{6}$ & $83 a$ & $1+1$ \\
3.12 & 79 & $2+2$ & $D_{10} \times D_{10}$ & $79 a$ & $1+1$ \\
3.14 & 57 & $2+1+1$ & $D_{6} \times 1 \times C_{2}$ & $57 b$ & $2+0+0$ \\
\hline
\end{tabular}

Heegner points arising from a Shimura curve parameterization

\begin{tabular}{cccccc}
3.15 & 35 & $2+1+1$ & $D_{6} \times C_{2} \times 1$ & $35 a$ & $1+1+0$ \\
\hline \multicolumn{5}{c}{ Stark-Heegner points over ring class fields of real quadratic fields } \\
4.1 & 145 & $2+1+1$ & $D_{8} \times C_{2} \times 1$ & $17 a$ & $1+1+0$ \\
\hline \multicolumn{5}{c}{ Points arising both as Heegner and Stark-Heegner points } \\
4.3 & 155 & $1+1+1+1$ & $1 \times C_{2} \times C_{2} \times C_{2}$ & $155 a$ & $1+0+1+0$ \\
4.3 & 155 & $1+1+1+1$ & $1 \times C_{2} \times C_{2} \times C_{2}$ & $155 c$ & $1+1+0+0$ \\
\hline
\end{tabular}

Points over fields cut out by the projective representations attached to exotic forms

\begin{tabular}{cccccc}
5.3 & 133 & $1+3$ & $1 \times A_{4}$ & $91 a, 91 b$ & $1+1$ \\
5.4 & 124 & $1+3$ & $1 \times A_{4}$ & $124 a$ & $1+1$ \\
5.4 & 124 & $1+3$ & $1 \times A_{4}$ & $26 b, 52 b$ & $0+2$ \\
5.5 & 283 & $1+3$ & $1 \times S_{4}$ & $4811 a$ & $2+0$ \\
5.6 & 148 & $1+3$ & $1 \times S_{4}$ & $37 a, 629 a, 629 d$ & $1+1$ \\
\hline
\end{tabular}

Points over abelian extensions of $\mathbb{Q}$

\begin{tabular}{cccccc}
7.1 & 57 & $1+1+1+1$ & $C_{3} \times C_{2} \times C_{3} \times C_{2}$ & $42 a$ & $1+0+1+0$ \\
7.2 & 21 & $1+1+1+1$ & $1 \times 1 \times C_{6} \times C_{6}$ & $26 a$ & $0+0+1+1$ \\
\hline
\end{tabular}

\section{References}

[AL78] A. O. L. Atkin and W. Li, 'Twists of newforms and pseudo-eigenvalues of W-operators', Invent. Math. 48(3) (1978), 221-243.

[BeDi] J. Bellaïche and M. Dimitrov, 'On the eigencurve at classical weight one points', Duke Math. J., to appear.

[BCDDPR] M. Bertolini, F. Castella, H. Darmon, S. Dasgupta, K. Prasanna and V. Rotger, ' $p$-adic $L$-functions and Euler systems: a tale in two trilogies', in Automorphic Forms and Galois Representations, Vol. 1, LMS Lecture Notes, 414 (Cambridge University Press, Cambridge, 2014), 52-102.

[BD1] M. Bertolini and H. Darmon, 'Kolyvagin's descent and Mordell-Weil groups over ring class fields', J. reine angew. Math. 412 (1990), 63-74.

[BD2] M. Bertolini and H. Darmon, 'Kato's Euler system and rational points on elliptic curves I: A p-adic Beilinson formula', Israel J. Math. 199 (2014), 163-188.

[BDP1] M. Bertolini, H. Darmon and K. Prasanna, ' $p$-adic Rankin $L$-series and rational points on CM elliptic curves', Pacific J. Math. 260 (2012), 261-303.

[BDP2] M. Bertolini, H. Darmon and K. Prasanna, 'Generalised Heegner cycles and $p$-adic Rankin L-series', Duke Math. J. 162(6) (2013), 1033-1148.

[BDR1] M. Bertolini, H. Darmon and V. Rotger, 'Beilinson-Flach elements and Euler Systems I: syntomic regulators and p-adic Rankin L-series', J. Algebraic Geom. 24 (2015), 355-378. 
[BDR2] M. Bertolini, H. Darmon and V. Rotger, 'Beilinson-Flach elements and Euler systems II: $p$-adic families and the Birch and Swinnerton-Dyer conjecture', J. Algebraic Geom. 24 (2015), 569-604.

[Br] E. H. Brooks, 'Generalized Heegner cycles, Shimura curves, and special values of $p$-adic $L$-functions', PhD Dissertation, University of Michigan, 2013.

$[\mathrm{Bu}]$ K. Buzzard, 'Computing weight one modular forms over $\mathbb{C}$ and $\overline{\mathbb{F}}_{p}$ ', in Computations with Modular Forms: Proceedings of a Summer School and Conference, Heidelberg, August/September 2011 (eds. G. Böckle and G. Wiese) (Springer, 2014), 129-146.

[Ch] T. Chinburg, 'Stark's conjecture for $L$-functions with first order zeroes at $s=0$, Adv. Math. 48 (1983), 82-113.

[CV] S. Cho and V. Vatsal, 'Deformations of induced Galois representations', J. reine angew. Math. 556 (2003), 79-98.

[Co] A. Cowan, 'Computational techniques for verifying the Birch and Swinnerton-Dyer conjecture for curves over cubic number fields', NSERC USRA (Undergraduate Summer Research Award) project, available at http://www.math.mcgill.ca/darmon /theses/cowan.pdf.

[Dar] H. Darmon, 'Integration on $\mathcal{H}_{p} \times \mathcal{H}$ and arithmetic applications', Ann. of Math. (2) 154(3) (2001), 589-639.

[DDLR] H. Darmon, M. Daub, S. Lichtenstein and V. Rotger, 'Algorithms for Chow-Heegner points via iterated integrals', Math. Comp. 84 (2015), 2505-2547. With an appendix by W. Stein.

[DLR1] H. Darmon, A. Lauder and V. Rotger, 'Gross-Stark units and $p$-adic iterated integrals attached to modular forms of weight one', Ann. Math. Québec, to appear. Volume dedicated to Prof. Glenn Stevens on his 60th birthday.

[DLR2] H. Darmon, A. Lauder and V. Rotger, 'Overconvergent generalised eigenforms of weight one and class fields of real quadratic fields', Adv. Math. 283 (2015), 130-142.

[DLR3] H. Darmon, A. Lauder and V. Rotger, 'Elliptic Stark conjectures and irregular weight one forms', in progress.

[DP] H. Darmon and R. Pollack, 'The efficient calculation of Stark-Heegner points via overconvergent modular symbols', Israel J. Math. 153 (2006), 319-354.

[DR1] H. Darmon and V. Rotger, 'Diagonal cycles and Euler systems I: a $p$-adic GrossZagier formula', Ann. Sci. Éc. Norm. Supér. 47(4), 47 p.

[DR2] H. Darmon and V. Rotger, 'Diagonal cycles and Euler systems II: the Birch and Swinnerton-Dyer conjecture for Hasse-Weil-Artin $L$-series', submitted.

[DR3] H. Darmon and V. Rotger, 'Elliptic curves of rank two and generalised Kato classes', submitted.

[DRS] H. Darmon, V. Rotger and I. Sols, 'Iterated integrals, diagonal cycles, and rational points on elliptic curves', Publ. Math. Besançon 2 (2012), 19-46.

[DFK] C. David, J. Fearnley and H. Kisilevsky, 'Vanishing of $L$-functions of elliptic curves over number fields', in Ranks of Elliptic Curves and Random Matrix Theory, London Mathematical Society Lecture Note Series, 341 (Cambridge University Press, Cambridge, 2007), 247-259.

[deS] E. de Shalit, Iwasawa Theory of Elliptic Curves with Complex Multiplication. p-Adic L-Functions, Perspectives in Mathematics, 3 (Academic Press, Inc., Boston, MA, 1987).

[DuLi] W. Duke and Y. Li, 'Harmonic Maass forms of weight one', Duke Math. J. 164 (2015), 39-113.

[Gh] E. Ghate, 'Ordinary forms and their local Galois representations', available for download at http://www.math.tifr.res.in/ eghate/math.html. 
[Gre] M. Greenberg, 'Stark-Heegner points and the cohomology of quaternionic Shimura varieties', Duke Math. J. 147(3) (2009), 541-575.

[Gro] B. Gross, 'On the factorization of $p$-adic L-series', Invent. Math. 57(1) (1980), 83-95.

[HaKu] M. Harris and S. Kudla, 'The central critical value of a triple product $L$-function', Ann. Math. (2) 133 (1991), 605-672.

[Hi1] H. Hida, 'Congruences of cusp forms and special values of their zeta functions', Invent. Math. 63 (1981), 225-261.

[Hi2] H. Hida, 'Elementary theory of L-functions and Eisenstein series', Lond. Math. Soc. St. Texts 26 (1993).

[Hi3] H. Hida, 'On the search of genuine $p$-adic modular $L$-functions for GL(n)', in Mémoires de la Société Mathématique de France, 2nd series, 67 (1996), 1-110.

[Ich] A. Ichino, 'Trilinear forms and the central values of triple product L-functions', Duke Math. J. 145(2) (2008), 281-307.

[Kato] K. Kato, ' $p$-adic Hodge theory and values of zeta functions of modular forms', in Cohomologies p-Adiques et Applications Arithmétiques. III, Astérisque, No. 295 (2004), ix, 117-290.

[Katz1] N. M. Katz, ' $p$-adic interpolation of real analytic Eisenstein series', Ann. of Math. (2) 104(3) (1976), 459-571.

[Katz2] N. M. Katz, 'p-adic L-functions for CM fields', Invent. Math. 49 (1978), 199-297.

[KW] C. Khare and J.-P. Wintenberger, 'Serre's modularity conjecture (I)', Invent. Math. 178 (2009), 485-504.

[La1] A. Lauder, 'Computations with classical and p-adic modular forms', LMS J. Comput. Math. 14 (2011), 214-231.

[La2] A. Lauder, 'Efficient computation of Rankin p-adic L-functions', in Computations with Modular Forms: Proceedings of a Summer School and Conference, Heidelberg, August/September 2011 (eds. G. Böckle and G. Wiese) (Springer, 2014), 181-200.

[Pr] D. Prasad, 'Trilinear forms for representations of GL(2) and local epsilon factors', Compos. Math. 75 (1990), 1-46.

[Si] J. H. Silverman, 'Computing rational points on rank 1 elliptic curves via $L$-series and canonical heights', Math. Comp. 68(226) (1999), 835-858.

[St1] H. M. Stark, ' $L$-functions at $s=1$. II. Artin $L$-functions with rational characters', Adv. Math. 17 (1975), 60-92.

[St2] H. M. Stark, 'Class fields and modular forms of weight one', in Modular Functions of One Variable, V (Proc. Second Internat. Conf., Univ. Bonn, Bonn, 1976), Lecture Notes in Mathematics, 601 (Springer, Berlin, 1977), 277-287.

[Wa] T. C. Watson, 'Rankin triple products and quantum chaos'. PhD Thesis, Princeton University, 2002, $81 \mathrm{pp}$.

[Wo1] M. Woodbury, 'Trilinear forms and subconvexity of the triple product $L$-function', submitted.

[Wo2] M. Woodbury, 'On the triple product formula: real local calculations', in preparation.

[YZZ] X. Yuan, S. Zhang and W. Zhang, The Gross-Zagier Formula on Shimura Curves, Annals of Mathematics Studies, 184 (Princeton University Press, Princeton, NJ, 2013).

[Zh] S.-W. Zhang, 'Gross-Zagier formula for GL(2). II', in Heegner Points and Rankin L-Series, Mathematical Sciences Research Institute Publications, 49 (Cambridge University Press, Cambridge, 2004), 191-214. 